\title{
Role of mechanosensitive ion channels in coordinated epithelial cell dynamics in Drosophila
}

\author{
Dissertation
}

for the award of the degree "Doctor rerum naturalium" at the Georg-AugustUniversity Göttingen

within the doctoral program "Genes and Development" of the Georg-August University School of Science (GAUSS)

Submitted by

\section{Prachi Richa}

born in Darbhanga, Bihar, India

Göttingen, Germany 2019 


\section{Members of the Thesis Committee}

$\begin{array}{ll}\text { Prof. Dr. Jörg Großhans } & \text { Department of Developmental } \\ \text { (Supervisor and first referee) } & \text { Biochemistry, } \\ & \text { Universitätsmedizin Göttingen, } \\ & \text { Germany }\end{array}$

Prof. Dr. Martin Göpfert

(Second referee)

Prof. Dr. Stefan Grill
Department of Cellular Neurobiology

Schwann-Schleiden Research Centre.

Göttingen, Germany

Biotechnology Center,

Technische Universität Dresden,

Germany

\section{Additional Members of the Examination Board}

Prof. Dr. Fred Wolf

Prof. Dr. Reinhard Schuh

Prof. Dr. Gregor Bucher
Theoretical Neurophysics, Max Planck Institute for Experimental Medicine, Göttingen, Germany

Department of Molecular

Developmental Biology, Max Planck Institute for Biophysical Chemistry, Göttingen, Germany

Department of Developmental Biology, Georg-August

University Göttingen, Germany

Date of oral examination: $\mathbf{0 2}^{\text {nd }}$ July 2019 


\section{Acknowledgements}

First, I would like to thank my supervisor, Prof. Dr. Jörg Großhans for providing me an opportunity to pursue my doctoral studies under his guidance. I am thankful to Jörg, for his valuable discussions and suggestions, and always being there to answer every little question. His optimistic viewpoint has been inspiring. Without his constant support and guidance, this project would not have been complete.

I would like to thank my thesis committee members Prof. Dr. Martin Göpfert and Prof. Dr. Stefan Grill for their guidance, and suggestions during the thesis committee meetings. I would like to thank Martin, for providing his valuable suggestions and reviews during meetings and discussions. I would like to thank my collaborators Prof. Dr. Fred Wolf, and Matthias Häring, for providing a detailed theoretical analysis. I would like to thank Fred, for his detailed discussions and valuable suggestions which provided an insight from a different perspective. I would also like to thank Matthias, for his contribution and efforts during this project.

I would like to express my gratitude to my colleagues and lab members for creating a friendly working atmosphere, and providing great discussions. I would like to thank Dr. Deqing Kong, who did important preliminary analysis for this project and also helped me solve many technical issues in day to day laboratory life. I am thankful to Deqing, for always being there as a friend and sharing his thoughtful advices. I thank Dr. Zhiyi Lv, Dr. Anja Schmidt, Long $\mathrm{Li}$, Dr. Boyang Liu, Dr. Maria Kriebel, and Dr. Shuling Yan, for discussing the data, results and sharing thoughts during our lab meetings and casual chit-chat.

I would like to thank GGNB and the Genes and development program for their commitment to higher education. I am thankful to the Department of Developmental Biochemistry, University of Göttingen, and SPP 1782 for funding this project. I would like to thank Manuela Manafas, and Johannes Sattmann, for their administrative and technical support.

Last, but not the least, I would like to thank my childhood best friend and beloved fiancé, Ratnakar Mishra, for his love and constant support during the entire journey of my Ph.D. Thank you Ratnakar, for constantly discussing my project, I will always cherish our everlasting scientific discussions. Thank you Ratnakar, for always being there in my life through thick and thin, your support means a lot to me. I would like to thank all my family members, especially my parents Mrs. Mala Jha and Mr. Ramesh Jha, for their constant support and believing in me through my entire educational career. I would like to thank my loving sisters, Divya, and Sammi, for their endless support and for always standing by my side. 


\section{Table of content:}

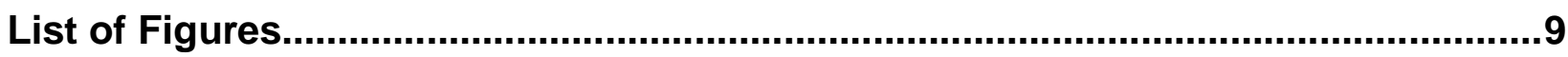

Abbreviations

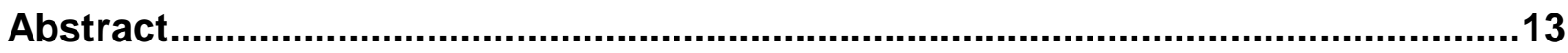

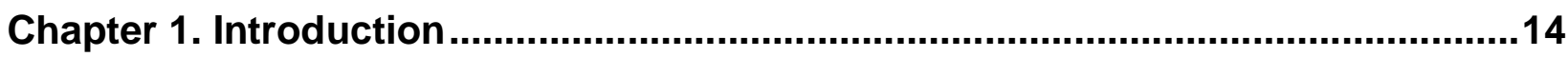

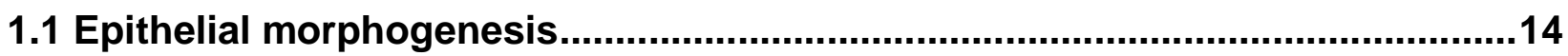

1.2 Apical constrictions: A common cell-shape change resulting in epithelial

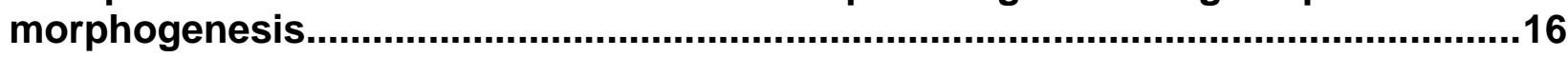

1.3 Dorsal closure as a model system to study coordinated epithelial

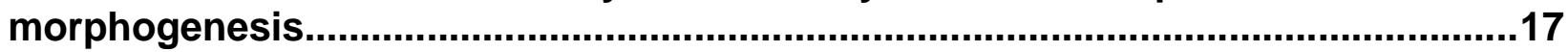

…… 18

1.3.1 Multiple tissue-scale forces during DC..................................................18

1.3.2 Non-autonomous oscillatory behavior in AS cells ...........................................19

1.4 Mechano-transduction and force-sensing at the adherens junction.................22

1.4.1 Force-dependent E-Cadherin clustering ..................................................23

1.4.2 Interplay between cell adhesion and actomyosin dependent contractility ..........24

1.4.3 Mechano-sensitive ion channels .............................................................25

1.5 Role of MS ion channels in mechano-transduction across different species ..26

1.5.1 Transmembrane channel-like protein (TMC) ………................................26

1.5.2 NOMPC (no mechanoreceptor potential C) ………..................................28

1.5.3 Mechanosensitive Piezo channels ............................................................29

1.6 Calcium signaling in epithelial morphogenesis ............................................30

1.6.1 Calcium signaling in Drosophila development ...........................................31

1.6.2 Calcium-dependent response to the epithelial wounding..................................33

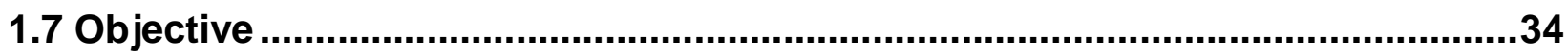

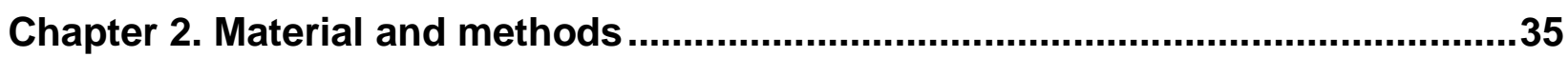

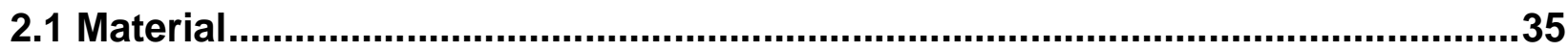

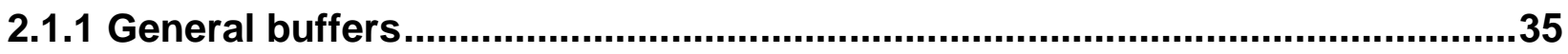

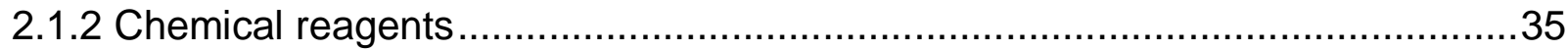

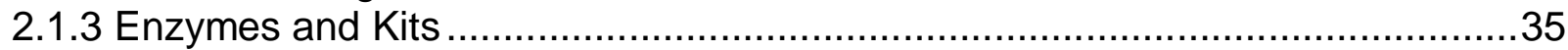

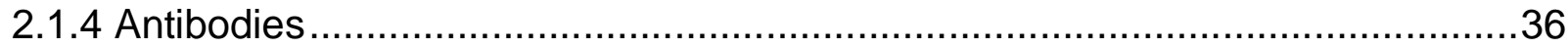

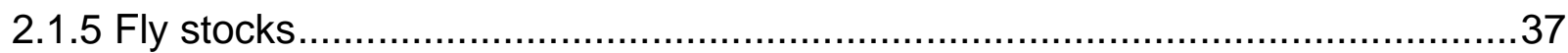

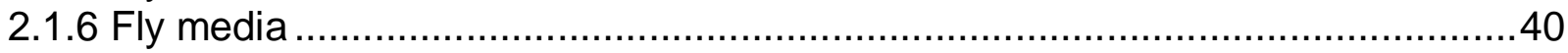

2.1.7 Media for bacterial culture.....................................................................

2.1.8 High Resolution Microscopy ................................................................... 41

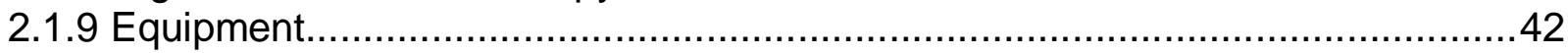

2.1.10 Other reagents and materials .............................................................. 43

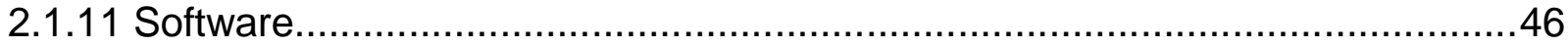

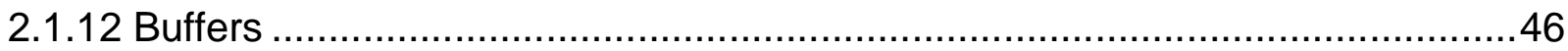

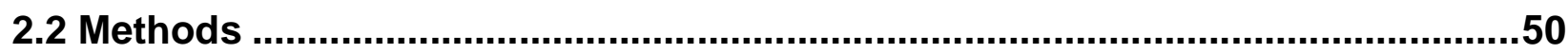

2.2.1 Genomic DNA extraction from adult flies ................................................50

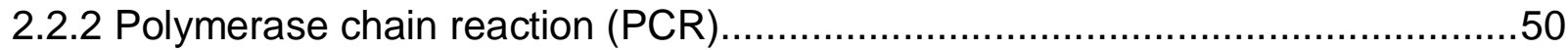

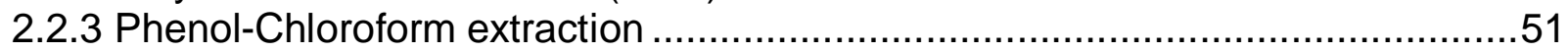

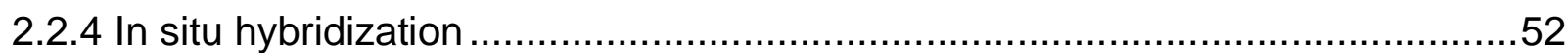


2.2.5 Western blot.

2.2.6 Co-Immunoprecipitation (Co-IP) with Tmc::eGFP and sepharose beads GFP binder...... .53

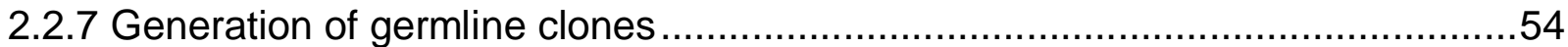

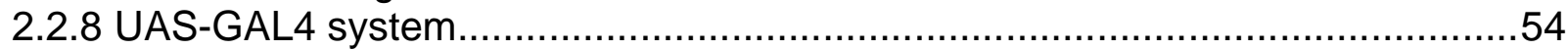

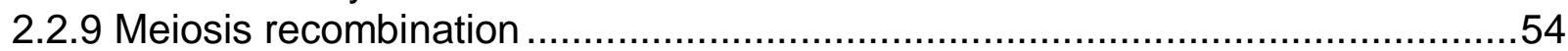

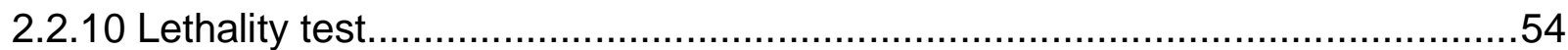

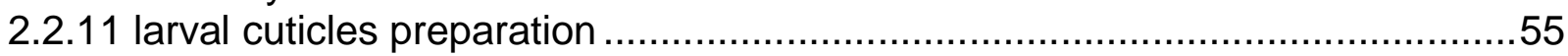

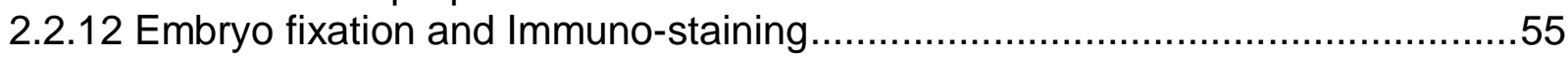

2.2.13 Permeabilization of vitelline membrane for Drug perturbation .......................56

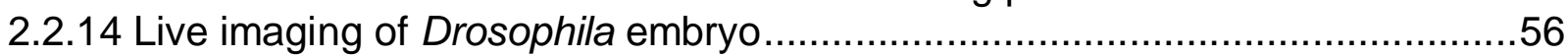

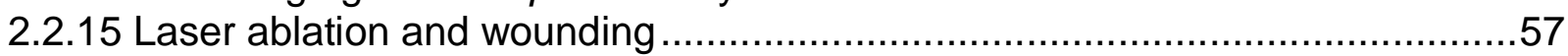

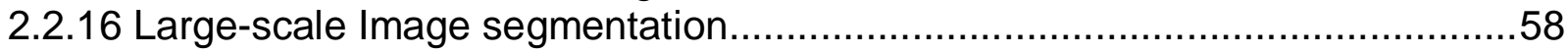

Chapter 3. Results..................................................................................................60

3.1 Expression of mechanosensitive ion channels in the AS epithelium ...............60

3.1.1 Expression of TMC, NOMPC, and Piezo in the epithelial cells during embryogenesis

3.2 Sub-cellular localization of ion-channels in the AS cells.

3.2.1 TMC is uniformly expressed at the apical and apico-lateral membrane in the AS cells

3.2.2 NOMPC localizes as clustered structures at the membrane in the AS cells ......62

3.2.3 Piezo localizes as clusters at the membrane and apico-medial region in the AS cells

3.3 TMC and NOMPC depletion lead to the embryonic lethality and cuticle defects in the embryo.

\subsection{Imaging and segmentation pipeline for large-scale computational image} analysis

3.5 TMC is involved in the maintenance of cell morphology and isotropy in the AS

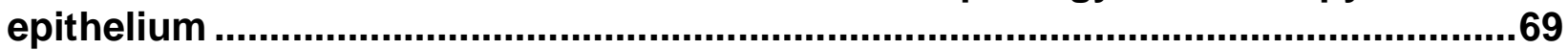

3.5.1 AS cells display anisotropic morphology in $T M C^{\Delta}$ and xit mutant embryos .......70

3.5.2 AS cell junctions display wavy morphology in $T M C^{\Delta}$ and xit mutant embryos ...73

3.5.3 Total number of AS cells are reduced in $T M C^{\Delta}$ and xit embryos .....................74

3.6 Coordinated oscillatory cell behavior and intercellular coupling between AS cell-pairs is affected in $T M C^{\Delta}$ and xit mutants.

3.6.1 Neighbor dependent, non-autonomous oscillations are faster in the AS cells in

$T M C^{\Delta}$ and $x i t$ compared to the wild-type. .75

3.6.2 Distribution of coupling types and intercellular coordination is affected in $T M C^{\Delta}$

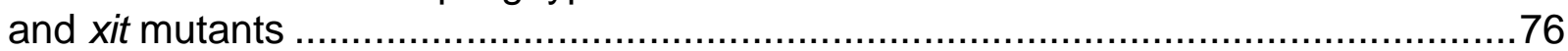

3.6.3 Spatial pattern of coupling types of AS cell-pair........................................ 78

3.7 AS cell junctions exhibit anisotropic force distribution in $T M C^{\Delta}$ and $x i t$ mutants .

3.7.1 Recoil velocity in the junctions with axial orientation...................................80

3.7.2 Increased recoil velocity in the laterally oriented junctions in $T M C^{\Delta}$ and xit mutants compared to the wild-type...

3.7.3 Anisotropic tension at the actin cable in the wild-type.

3.8 Neighbor dependent $\mathrm{Ca}^{2+}$ mediated wound response is perturbed in $T M C^{4}$ and xit mutant

3.8.1 $\mathrm{Ca}^{2+}$ dependent Myosin II accumulation following epithelial wounding. .84 
3.8.2 $\mathrm{Ca}^{2+}$ dependent neighbor cell response to wounding is perturbed in $T M C^{4} \ldots . . .86$ 3.8.3 Neighbor dependent $\mathrm{Ca}^{2+}$ response to wounding is severely perturbed in xit....86

3.9 Increasing extracellular $\mathbf{M g}^{2+}$ induces morphogenetic defects in AS cells.......88

3.10 NOMPC depletion results in overall decrease in junctional tension compared

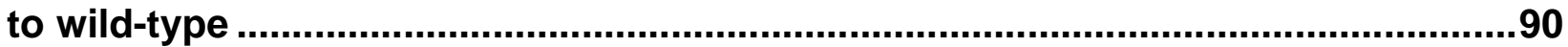

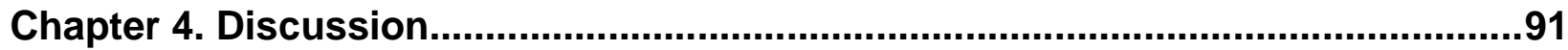

4.1 Mechanosensitive ion channels are expressed in the AS epithelial cells in Drosophila

4.1.1 TMC, NOMPC, and Piezo display specific sub-cellular localization in the AS cells

4.1.2 Role of TMC and NOMPC during embryogenesis

4.2 TMC and xit are involved in the regulation of isotropic force balance within the AS .93

4.2.1 Maintenance of isotropic morphology of the AS cells during early DC is dependent on TMC and xit

4.2.2 TMC and xit maintain isotropic tension distribution in the AS cells

4.3 TMC is an essential component in the pathway establishing coordinated cell behavior in the AS.

4.3.1 TMC and xit regulate oscillatory dynamics in AS cells ..................................97

4.3.2 TMC mediated mechanotransduction is essential for the distribution of positively coupled cell-pairs within the AS tissue.

4.4 TMC play a role in $\mathrm{Ca}^{2+}$ dependent response of the neighboring cells to wounding. 100

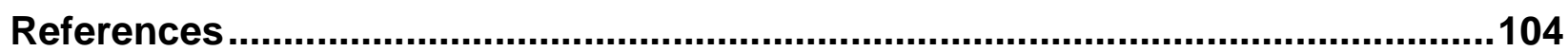

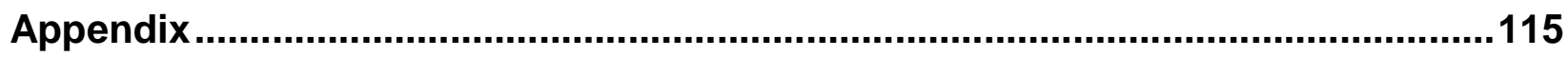

Appendix 1: Anisotropy of AS cells (single cases) ...........................................116

Appendix 2: Displacement trajectories of junctions (single cases) ....................117

Appendix 3: Increase in $\mathrm{Ca} 2+$ signal in the neighboring cells following wounding (single cases)

Appendix 4: Spatial distribution of positive and negative coupling type of AS cell pairs.

Appendix 5: F-actin staining in WT and $T M C^{\perp}$ mutant embryos 


\section{List of Figures}

Fig 1.1 Coordinated cell behavior during tissue-scale morphogenetic events in Drosophila embryo

Fig 1.2 Mechanism and function of apical contractile cell behavior

Fig 1.3 Morphogenesis at cellular levels and tissue-scale during DC in Drosophila embryos

Fig 1.4 Forces acting during DC.

Fig 1.5 Pulsed contractions in AS cells.

Fig 1.6 AS pulses are coupled and require tissue tension to oscillate

Fig 1.7 E-Cadherin clusters at the cell-cell junction.

Fig 1.8 Adhesion complex at the epithelial junction.

Fig 1.9 Model of MGC response to mechanical stimuli.

Fig 1.10 Transmembrane channel-like protein.

Fig 1.11 Mechanotransduction via NOMPC.

Fig 1.12 Structure and conformation of Piezo1.

Fig 1.13 Calcium signaling dependent cell contractions.

Fig 1.14 wound repair in Drosophila epithelium.

Fig 3.1 Expression of mechanosensitive ion channels in AS epithelium.

Fig 3.2 Cellular localization of TMC in the AS cells.

Fig 3.3 Cellular localization of NOMPC in the AS cells.

Fig 3.4 Cellular localization of Piezo in the AS cells.

Fig 3.5 Cuticle phenotype.

Fig 3.6 Deep learning neural network-based Segmentation approach.

Fig 3.7 Loss of epithelial integrity in unhatched $T M C^{\Delta}$ embryos. 
Fig 3.8 Quantification of spatiotemporal change in anisotropy of the AS cells from germ-band retraction to early DC stage.

Fig 3.9 AS cells in $T M C^{\Delta}$ and xit embryos remain anisotropic post-germband 71 retraction.

Fig 3.10 Quantification for cell junction waviness.

Fig 3.11 Total cell-count in the AS.

Fig 3.12 Oscillatory dynamics of AS cells in wild-type, $T M C^{\Delta}$, and xit mutant.

Fig 3.13 Intercellular coupling between AS cell-pairs in wild-type, $T M C^{\Delta}$, and xit mutant.

Fig 3.14 Spatial 2D map of cell coupling types in AS in the wild-type, $T M C^{\Delta}$, 78 and xit embryo.

Fig 3.15 Quantification of junctional recoil velocity of the AS cells.

Fig 3.16 Recoil velocity at the axial junctions within the AS cells in the wildtype, $T M C^{\Delta}$, and xit embryos.

Fig 3.17 Increased recoil velocity at the laterally oriented junctions of the AS cells in $T M C^{\Delta}$ and xit compared to wild-type embryos.

Fig 3.18 Anisotropic tension distribution at the AC.

Fig 3.19 UV laser induced wounding triggers $\mathrm{ca}^{2+}$ dependent response in the neighbor cells in the AS tissue.

Fig 3.20 Wound induced, neighbor dependent $\mathrm{Ca}^{2+}$ dynamics is perturbed in $T M C^{\Delta}$, and xit mutant.

Fig 3.21 $\mathrm{Ca}^{2+}$ dynamics in xit mutant.

Fig 3.22 $\mathrm{MgCl}_{2}$ treatment induces morphological defects in the AS.

Fig 3.23 NOMPC depletion affects junctional morphology and tension.

Fig 4.1 TMC and xit plays a role in the maintenance of the uniform force distribution within the AS tissue.

Fig 4.2 TMC and xit dependent mechanotransduction distinctly control the coordination of the coupled cell pairs.

Fig 4.3 Insight from functional mechanism of TMC in the auditory system. 


\section{Abbreviations}

AS

DAPI

DC

DNA

$\mathrm{KO}$

MS

NOMPC

PBS

PCR

RNA

RNAi

$\mathrm{ROI}$

rpm

Spg-Sqh

SDS

TMC

TRP
Amnioserosa

4',6-diamidino-2-phenylindole

Dorsal closure

Deoxyribonucleic acid

Knock-out

Mechanosensitive

No mechanoreceptor potential C

Phosphate buffer saline

Polymerase chain reaction

Ribonucleic acid

RNA interference

region of interest

rotations per minute

Spaghetti squash

Sodium dodecyl sulfate

Transmembrane Channel-like protein

Transient receptor potential 


\section{Abstract}

Epithelial cells are able to sense and mechanically respond to the forces and movements generated and transmitted through their neighbors. These forces are multicellular at the tissue level, and are transmitted via multimeric protein complexes at the cell adhesion sites between two neighboring cells. The cell membrane in epithelial cells serve as a major site for receiving external mechanical forces, and via mechano-transduction these stimuli are converted into intracellular biochemical signals. Previous understanding of the junctional adhesion complexes (cadherin/catenin complex) may provide an insight, yet the key molecular components of mechano-transduction remained unidentified so far. In this study, we hypothesize that mechanosensitive (MS) ion channels (TMC, NOMPC and Piezo), might respond to the changes in tension at the plasma membrane and are crucial for maintenance and establishment coordinated cell behavior.

A major morphogenetic transformation event in Drosophila embryos is Dorsal closure (DC). This event critically depends on the dynamics of a squamous epithelium called the Amnioserosa (AS). AS cells exhibit dynamic mechanical behavior that appears to be coordinated between cells by undergoing stable oscillations between cross-sectional area. In addition, AS cells are highly accessible to genetic manipulations and quantitative live cell imaging. This provides a robust model system to study mechanosensing and mechanotransduction in epithelial cells.

Changes in tension at the adherens junction could be detected via mechano-transduction pathways, allowing cells to communicate with each other. I have found that MS ion channels are expressed in AS epithelium and are involved in establishing coordination between AS cells. In particular, I have found that the putative ion channel TMC is required for achieving isotropic junction tension and morphology within the AS. In order to understand the potential role of MS ion channels in establishing this coordinated cell behavior, I established methods and assays to follow $\mathrm{Ca}^{2+}$ dynamics and turnover in the AS cells.

This study could reveal and characterize previously unanticipated functions of MS putative ion channel TMC in epithelial tissue morphogenesis. I attempted to elucidate the principles by which the interaction of adherens junctions and MS ion channels may contribute to guide the active mechanical behavior in the epithelial cells. 


\section{Chapter 1. Introduction}

\subsection{Epithelial morphogenesis}

Morphogenesis is derived from the Greek word gennisi (generation) and morphê (shape). It is the process of how an organism, is shaped at every stage of its growth and development starting from the fertilized egg (Keller, 2012). Tissue-scale morphogenesis and cell shape changes are crucial for driving major developmental processes, such as gastrulation, tube formation, neurulation, to name a few. Many morphogenetic events involve coordinated movement of group of cells, in order to shape a tissue during development.

A

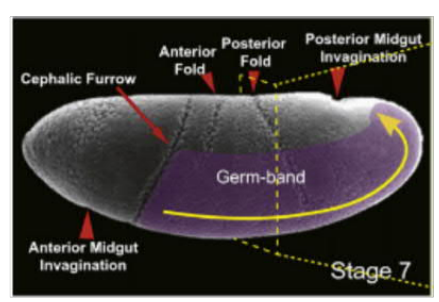

B

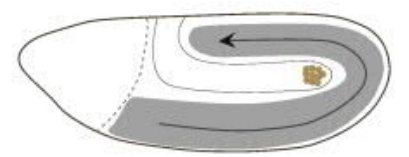

Germ(band-extension

C

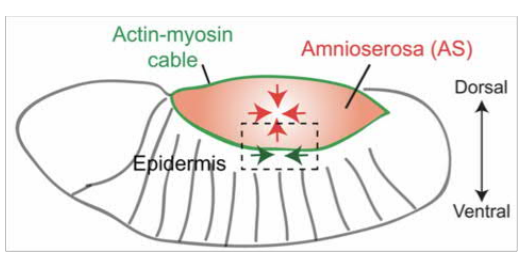

$A^{\prime}$

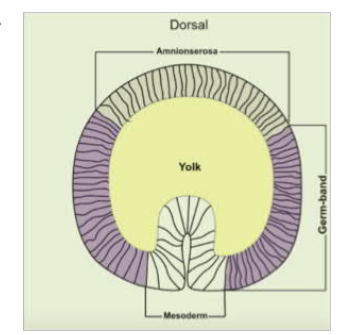

$B^{\prime}$

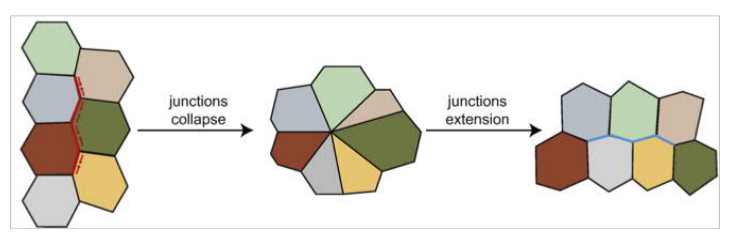

$C^{\prime}$

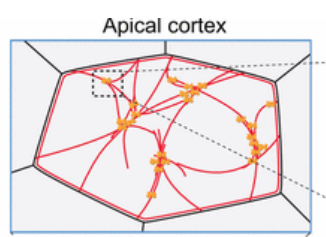

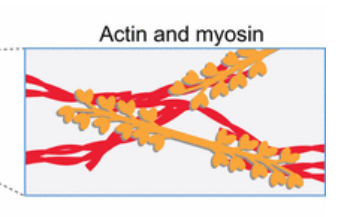

Fig 1.1 Coordinated cell behavior during tissue-scale morphogenetic events in Drosophila embryo. (A$\left.A^{\prime}\right)$ Apico-basal constriction of the ventral cells driving mesoderm invagination during Gastrulation. (B-B') Cell intercalation during germ-band extension. (C-C') Apical constrictions in amnioserosa cells during dorsal closure. Figure modified from (Kong et al., 2017) and (Martin and Goldstein, 2014).

In the Drosophila melanogaster, some of the well-characterized morphogenetic processes correspond to gastrulation (Leptin and Grunewald, 1990), germ-band extension (Kong et al., 2017), and dorsal closure (DC) (Jacinto et al., 2002) among others. These tissue-scale morphogenetic events are largely the consequence of continuously regulated and coordinated behavior of a group of cells via complex mechanisms (Fig 1.1) (Kiehart et al., 
2000b; Heisenberg and Bellaïche, 2013; Gilmour et al., 2017). One of the many morphogenetic events employing coordinated cell behavior occurs during gastrulation in Drosophila. During this process, the apical surface of a stripe of ventral cells constituting the mesoderm constricts, modifying their length along their apico-basal axis, while neighboring cells remain unchanged in their morphology. This further drives mesoderm invagination (Fig 1.1, A-A'). (Kölsch et al., 2007).

Another example of coordinated cell behavior in tissue-scale morphogenesis is during germband extension. At this stage, the embryo extends up to two-fold along the anterior-posterior axis. Major contribution to drive this tissue scale morphogenetic event is provided by cell intercalation, where a group of cell are able to modify their relative position within the tissue, by transiently shortening their cell junctions (Kong et al., 2017) (Fig 1.1, B-B').

Dorsal closure is one of the major morphogenetic events during embryonic development of Drosophila. During dorsal closure, coordinated tissue rearrangements occur in order to drive the lateral epidermis dorsally in a cohesive manner. Additionally, this leads to the displacement of extra-embryonic, squamous epithelial tissue called Amnioserosa (AS), in order to fully envelope the embryo and for continuous epithelium. In order to drive this process, coordinated apical constrictions of the AS cells significantly contribute to the generation of tissue-scale forces (Fig 1.1, B-B') (Kiehart et al., 2000a; Martin and Goldstein, 2014).

Previous studies in the field collectively indicate that the spatiotemporal control and regulation of local cell behavior of a group of cells is crucial in order to determine the shape of the tissue. Regulation of cell behavior of a group of cells relies on complex mechanisms including morphogens, signaling pathways and mechanical forces (Gilmour et al., 2017). One of the most intriguing questions, in order to completely understand the cellular and tissue-scale morphogenetic abilities of the organisms, is how different cellular mechanisms are coordinated and regulated while performing collective cell movements, with the motive of shaping the organism (Keller, 2012). 


\subsection{Apical constrictions: A common cell-shape change resulting in epithelial morphogenesis}

Within the epithelial tissue, highly coordinated dynamic movements and deformations shape the embryo (Quintin et al., 2008) (see Fig 1.1). Coordinated apical constrictions of group of cells are central to some key developmental events occurring throughout the metazoa. In many organisms, epithelial cells undergo apical constrictions generally at early stages of embryogenesis, such as during gastrulation in Caenorhabditis elegans (Rohrschneider and Nance, 2009), during gastrulation and dorsal closure in Drosophila melanogaster (Sawyer et al., 2010; Sokolow et al., 2012), as well as during vertebrate neural tube formation, among others (Andrew and Ewald, 2010).

A Myo-Il dynamics during apical constriction

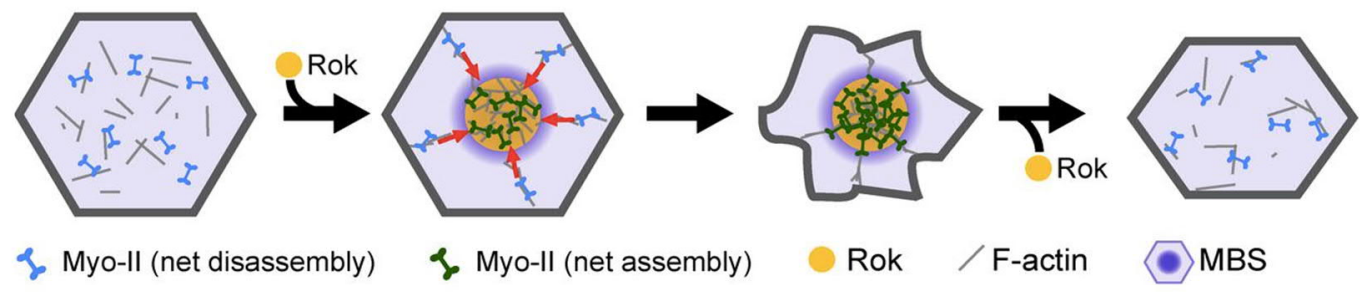

B Pulsatile constriction

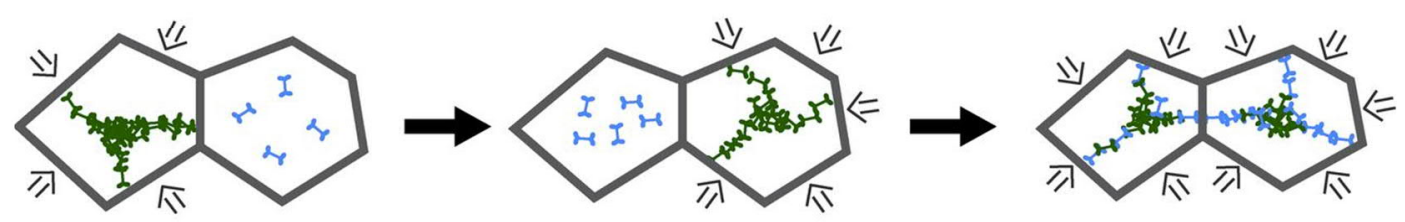

Continuous constriction

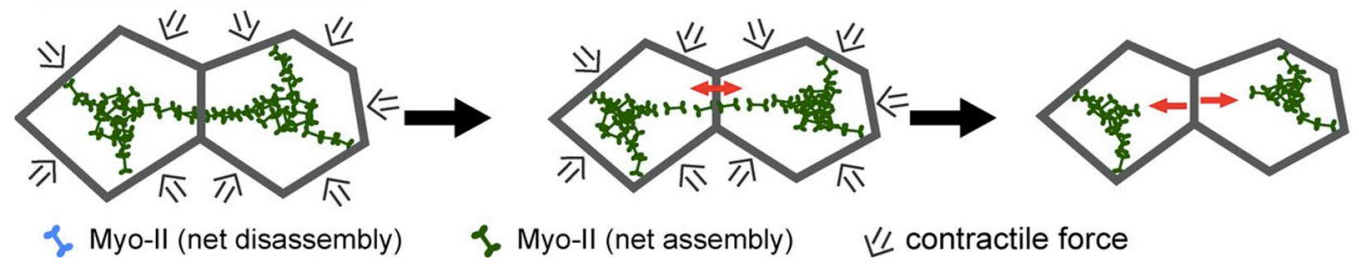

Fig 1.2 Mechanism and function of apical contractile cell behavior. (A) Myo-II dynamics during apical cell constriction. The contractile pulse is initiated by Rok dependent Myo-II phosphorylation and activity. (B) MyoII assembly during pulsatile contractions: Myo-II pulses induce contraction asynchronously in the adjacent cells. In addition, Myo-II remodeling allows cells to adjust contacts to maintain stable intercellular cytoskeletal connections. On the other hand, Continuous Myo-II assembly decreases the ability of actomyosin networks to dynamically adjust to changes in tissue mechanics, resulting in loss of intercellular connections of Myo-II structures. Figure adapted from (Vasquez et al., 2014). 
In epithelial cells, actomyosin contractility play a crucial role during continuous apical contractions. Previous studies suggest that the sub-cellular organization of contractile cytoskeletal networks facilitates force generation., senses and transmits forces between the cells and therefore better understanding for coordinated cell behavior. During tissue-scale morphogenetic events, each cell should be able to mechanically sense and respond to the external forces in order to participate effectively in controlling the cellular and tissue remodeling. Continuous expansion or contraction of plasma membrane domains during apical constriction requires changes in membrane tension in single cells or in a group of cells. Dynamic regulation of cell-cell adhesion at the junction between two cell-borders play a crucial role in cell rearrangements during these events (Gumbiner, 1996).

Apical constriction requires the apical and junctional recruitment and remodeling of an actomyosin contractile network. Non-muscle Myosin II pulses have been shown to correlate with transient fluctuations and change of cell shape and area in epithelial cells (Fig 1.2). In the Drosophila epithelium, phosphorylation of the regulatory light chain (Myo-IIRLC) by Rok activates Non-muscle Myosin-II (Dawes-Hoang et al., 2005; Vasquez et al., 2014). Rok is activated by the RhoGTP exchange factor RhoGEF2 and RhoA (Kölsch et al., 2007). During apical constriction, the apical actomyosin network predominantly flows towards the apicomedial region of the cortex in order to drive constriction of the apical surface of the cells (Fig 1.2, A and B) (Martin and Goldstein, 2014).

\subsection{Dorsal closure as a model system to study coordinated epithelial morphogenesis}

Large tissue-scale movements drive the shaping of the embryo during development. Dorsal closure (DC) is one of the major morphogenetic events during early embryogenesis in Drosophila. It has been well studied with respect to the force distribution intrinsic and extrinsic to the tissue in order to drive morphogenesis (Kiehart et al., 2000a; Hutson et al., 2003; Solon et al., 2009). DC occurs 8-12hr after the egg lay, corresponding to the embryonic stages 13-15 (Campos-Ortega and Hartenstein, 1997). In a preceding event to DC, germ-band retraction takes place. During germ-band retraction, the extended germband (extending from the ventral and posterior side of the embryo to its dorsal side, post gastrulation stage) now retracts back to the posterior end of the embryo. The retraction 
causes exposure of a hole on the dorsal side of the embryo that is filled with an extraembryonic squamous epithelial tissue called Amnioserosa (AS) (Fig 1.3).

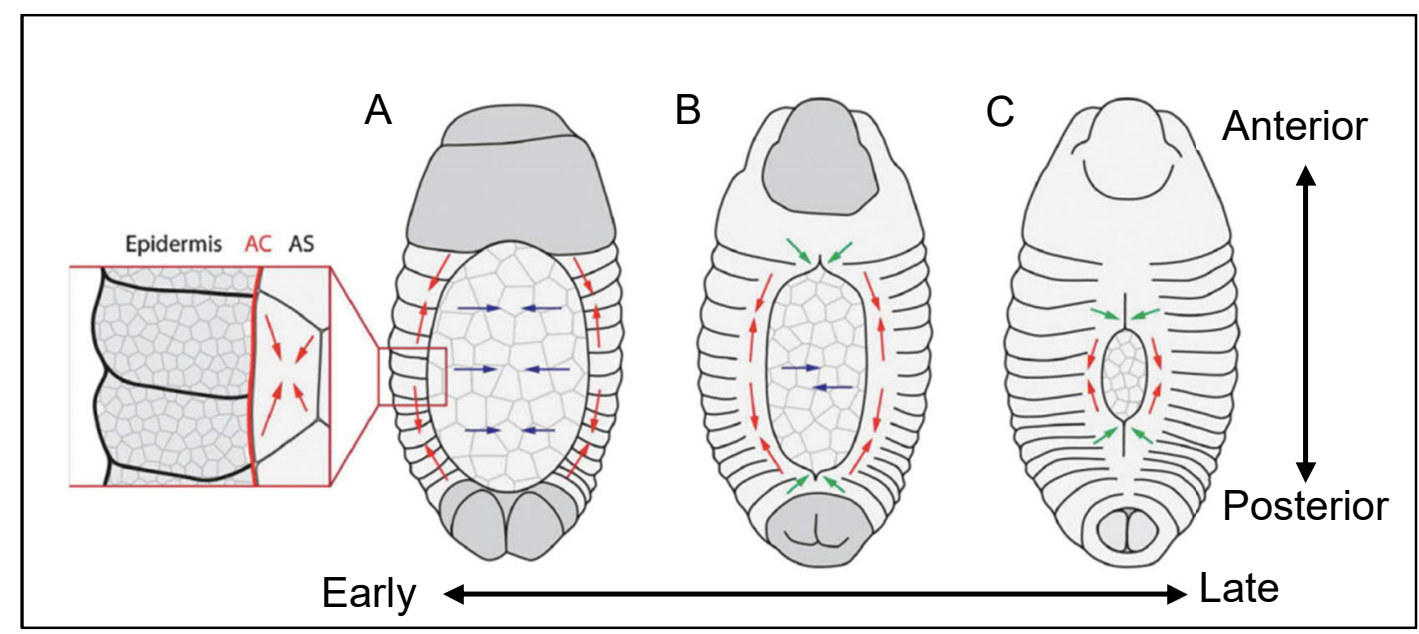

Fig 1.3 Morphogenesis at cellular levels and tissue-scale during DC in Drosophila embryos. (A) AS cells undergo continuous apical constriction, reflecting oscillatory behavior. (B) Leading edges meet at the canthi of the opening. (C) Zippering forces (green arrows) drive the closing of the dorsal hole. Figure adapted from (Heisenberg, 2009). Red arrows indicate contractile forces acting at individual AS cell (shown in the inset figure) and at the leading edge of the epidermis (from early to late panels).

\subsubsection{Multiple tissue-scale forces during DC}

The two highlights of DC event are (1) Continuous pulsatile constriction of the apical surfaces of AS cells, which contribute to the forces driving DC (Fig 1.3, A) (Pasakarnis et al., 2016). (2) The movement of supra-cellular actin cable (AC), located at the leading edges (anterior-most lateral epidermis) on either side of the opening, towards the midline in order to seal the dorsal gap and form a continuous epithelium (FIG 1.3, B, C) (Jacinto et al., 2002). At the end of the DC, following the dorsal-ward movement of the leading edges, AS cells ingress below the epidermal cell sheets and undergo apoptosis (Toyama et al., 2008).

Number of studies, exploring the cellular and tissue-scale mechanisms and signaling pathways during DC, suggest that a coordinated interplay of forces generated within the two tissue, the lateral epidermal cells and the AS cells, is essential for driving this event (Fig 1.4) (Solon et al., 2009). First, the AS cells exert active actomyosin dependent contractile forces via continuous oscillations, pulling on the two edges of the lateral epidermis together at the cathus. Continuous pulsatile contractions of AS cells are essential to drive this process, as the disruption of cell contractility lead to failure of the DC event (Kiehart et al., 2000a; 
Pasakarnis et al., 2016). In addition to this, there are resistive forces being generated from the epidermal cells surrounding the AS tissue in order to counteract the contractile forces. These forces at the epidermal front were revealed via laser micro-ablation of the cells at the leading edge, along the epidermis-AS boundary. Laser dissections induces fast retraction of the lateral epidermis revealing the high tension underlying at the epidermal-AS boundary (Fig 1.3 inset image, B, Fig 1.4) (Hutson et al., 2003).

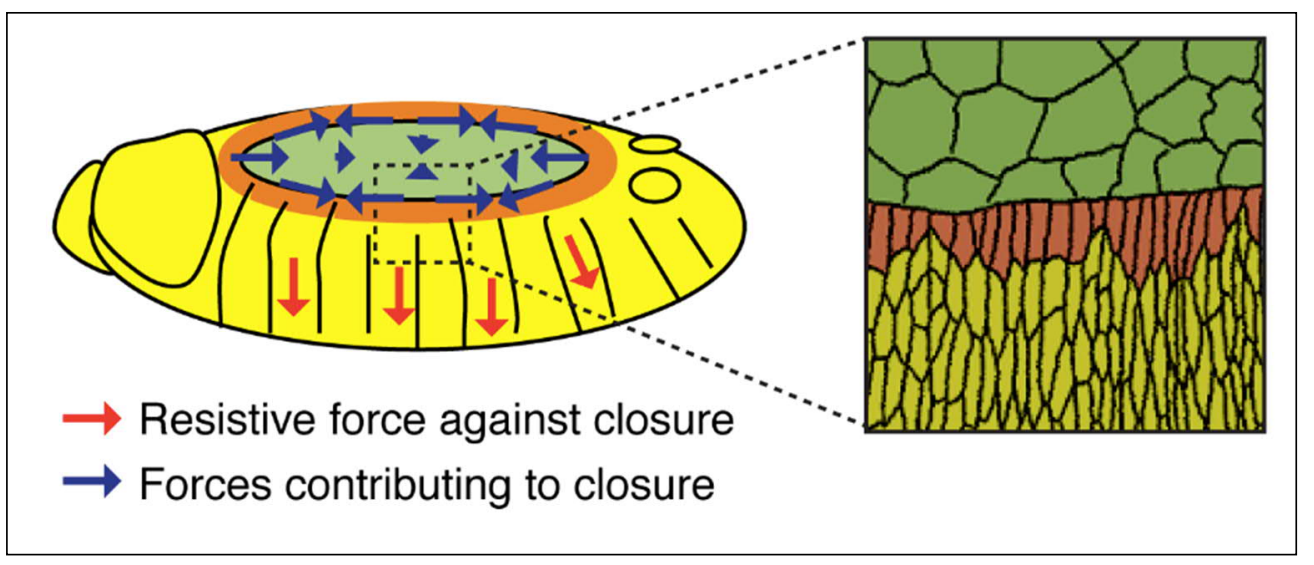

Fig 1.4 Tissue-scale forces during DC. Schematic showing key morphogenetic events at the cellular level and tissue-scale during DC in Drosophila embryo. AS (green) and the AC are able to generate contractile forces (blue arrows) and the lateral epidermis (yellow) generate tension counteractive (orange) to the contractile forces. Figure adapted from (Mateus and Arias, 2011).

In addition to the different forces originating from the AS and the lateral epidermis, tensile forces are generated by a highly dynamic, supracellular actomyosin cable (AC), which forms around the dorsal opening and at the border between the leading epidermis and AS tissue. The actomyosin cable is thought to behave as a purse string that contracts and shortens the leading edge, eventually bringing the two epidermal fronts together (Fig 1.3, Fig 1.4). During the late phase of $D C$, dynamic filopodial protrusions on the $A C$ of the opposing leading edges intertwine, in order to form a tight seam at the dorsal midline. This completely seals the dorsal gap and give rise to a continuous epithelium (Kiehart et al., 2000a; Hutson et al., 2003; Heisenberg, 2009).

\subsubsection{Non-autonomous oscillatory behavior in AS cells}

A central player of DC is the highly dynamic and morphogenetic AS tissue. AS cells undergo rapid and continuous cycles of cell surface area oscillations, which is crucial in the dorsalward displacement of the neighboring lateral epidermis. Individual AS cells continuously contract their apical surfaces with a periodicity of $230 \pm 76 \mathrm{sec}$ (Fig 1.5, A) (Solon et al., 2009; 
Blanchard et al., 2010). The major driving force for the apical contraction and expansion come from the cycles of assembly and disassembly of cortical actomyosin networks (Fig 1.5, B). Sqh-GFP (GFP fused to the regulatory light chain of non-muscle Myosin II: spaghettisquash) localize as two distinct structures at the apices of the cells. Myosin II localizes as

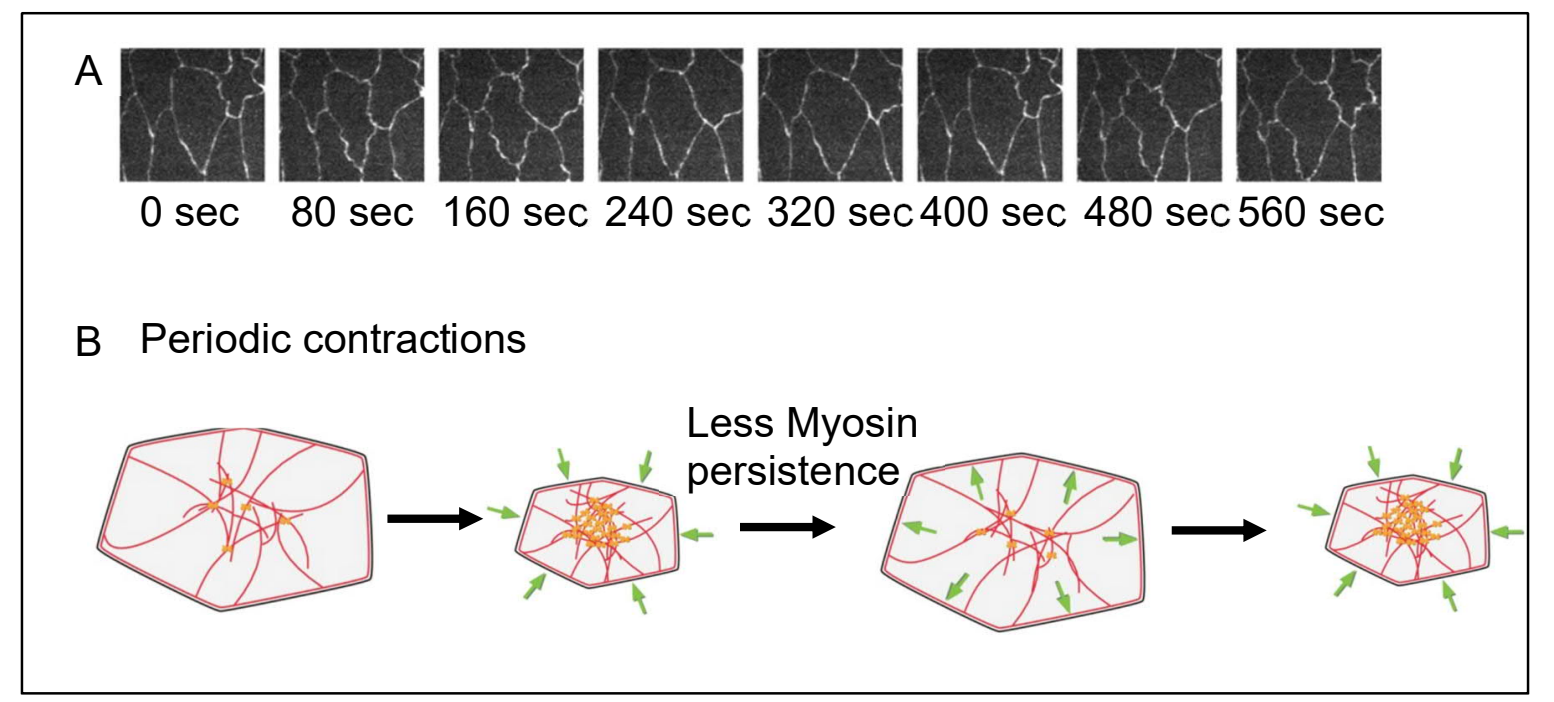

Fig 1.5 Pulsed contractions in AS cells. (A) 45 mins to 1 hour prior to the initiation of the DC, AS cells, undergo apical surface fluctuations with a periodicity of $230 \pm 76 \mathrm{sec}$. (B) Cell shape fluctuations are result of contractions of the apical surface area without stabilization, between pulses. Figure modified from (Solon et al., 2009; Martin and Goldstein, 2014).

dynamic apico-medial network, and at cell-cell adherens junctions forming a cortical ring. It has been shown that Myosin II transiently accumulate and disperse as a wave across the apical surface of cells. The formation of these Myosin II foci coincided with the local displacements of the membrane towards the foci. This further suggest a contribution of the apico-medial pool of Myosin II in driving the cell shape fluctuations (Fig 1.5, B) (Hutson et al., 2003; Blanchard et al., 2010; Lynch et al., 2013; Martin and Goldstein, 2014; Pasakarnis et al., 2016)

AS cell pulsing appears to be non-autonomous and coupled with the neighboring cells. It has been shown that the pulsation pattern of the direct neighbors was repeatedly indicated to be an in-phase or anti-phase correlation (Fig 1.6, A). This coupling fades with distance as the oscillations of cells further apart does not seem to be affected. Furthermore, when laserinduced cuts are made at a single junction connecting two AS cells, the neighboring cells respond with a relaxation of the surrounding tissue. The AS cells adjacent to the opening, that are in direct contact with the ablated target junction, stop their oscillatory behavior 
almost instantaneously. This provides stronger evidence for coordination and coupling between neighboring cells (Solon et al., 2009; Jayasinghe et al., 2013).

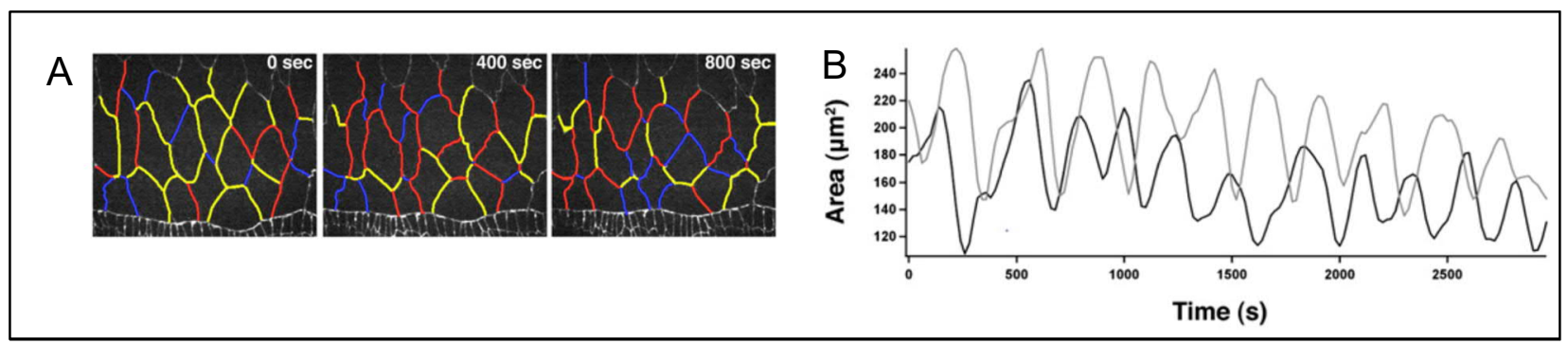

Fig 1.6 AS pulses are coupled during dynamic oscillations. (A) Cross-correlation map of neighboring AS cells over three to four full oscillation phases (blue boundaries: in-phase correlations, red boundaries: antiphase correlations, and yellow boundaries: cells switching between the two). (B) Short phase correlation of apical surface area fluctuations in two neighboring AS cells of over time. Figure adapted from (Solon et al., 2009).

These experiments (Fig 1.6 A, B) strongly suggest that the oscillatory behavior AS cell are neighbor cell-dependent and temporally coordinated. It also suggests that in order to maintain balance in the tissue tension there is a requirement of a mechanism based on mechanosensing and mechanical feedback within the tissue. Coordination and coupling between the neighbors within the AS, via adherens junction, might provide a mechanism for this. 


\subsection{Mechano-transduction and force-sensing at the adherens junction}

Physical forces play a major role in cell shape change and morphogenesis of epithelial cells. Mechanical forces experienced by the cells could be either external, exerted via the neighboring cells in the tissue, or internally induced by the intracellular actomyosin driven contractility. These forces once transmitted, are sensed by the cell. This force transduction and sensing could be understood as mechanotransduction: transduction of mechanical stimuli into biochemical signals, which enables the cell to sense and respond (Orr et al., 2006). Understandings from the previous research show the involvement of mechanosensitive ion channels, integrins, cadherins, actomyosin networks, and several other signaling molecules in mechano-transduction at the cell junctions. However, how cells respond and transmit forces and adapt to the mechanical properties of neighboring cells remained vaguely understood so far.

Epithelial cells interact with each other and their physical environment primarily via cell-cell adhesion, which is E-Cadherin dependent in the epithelium. Cell-cell communication and coordination could be established within the epithelium via (1) exchange of biochemical signals such as secreted signaling ligands, and (2) via the ability of the cells to respond to mechanical stimulus. Cells in epithelial tissue are physically coupled to each other via intercellular junctions and forces are transmitted across the tissue and as well as between connected neighboring tissues (Borghi et al., 2012; Maître and Heisenberg, 2013). Adherens Junctions function as a platform to sense changes in tension exerted by the adjacent cells (Matsuzawa et al., 2018). E-Cadherin in epithelial cells facilitates homophilic adhesion between the cells. The intracellular domain of E-Cadherin binds to the adaptor proteins $\beta$ catenin and $\alpha$-catenin and form an adhesion complex and links adhesion receptors to the underlying actin cortex (Fig 1.8, A). These complexes serve as a major site for receiving mechanical stimuli in the cell (Niessen et al., 2011) (Guillot and Lecuit, 2013; Chanet and Martin, 2014). Based on these previous understanding, conceivable mechanisms concerning the mechano-transduction and force sensing via adherens junctions are discussed in following subsection:

\subsubsection{Force-dependent E-Cadherin clustering.}

\subsubsection{Force-dependent conformational changes in $\alpha$-Catenin,}

\subsubsection{Mechano-sensitive ion channels.}




\subsubsection{Force-dependent E-Cadherin clustering}

E-cadherin mediated trans-homodimerization and cis-interactions results in lateral clustering. In the epithelium, the maintenance and stability of these E-cadherin clusters require interaction with actin. E-cadherin clusters at the adherens junction (AJ) form an adhesive belt that mechanically holds and couple two epithelial cells together. Clustering and de-clustering of E-Cadherin provide cells the ability to deform and remodel cell contacts and eventually might transduce tension to control local junction dynamics (Truong Quang et al., 2013).

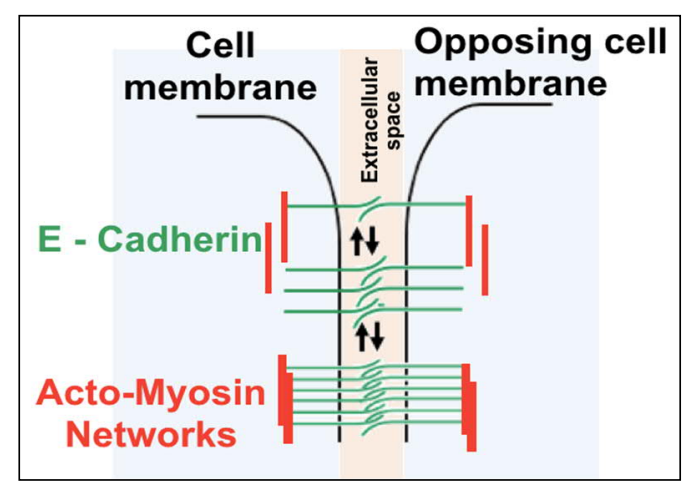

Fig 1.7 E-Cadherin clusters at the cell-cell junction. Schematic representation of tension dependent clustering of E-Cadherin and actomyosin reorganization at the adherens junction.

$\mathrm{N}$-glycosylation (Post-translational modification for membrane proteins) is crucial for distribution and clustering of E-Cadherin within the plasma membrane and at the adherens junctions. One of the genes contributing to E-cadherin glycosylation is xit. xit encodes for one of the conserved enzymes involved in the addition of the terminal glucose residues to the glycan precursor. Among others, E-Cadherin may be one of the major targets of xit during early morphogenetic events like the germ-band extension. In xit depleted embryos intracellular distribution and clustering of E-Cadherin seem to be affected, although the total amount of E-Cadherin protein is not affected. Also, during germ-band extension morphogenetic rearrangement of cells via cell intercalation in the lateral epidermis is impaired in xit mutants (Zhang et al., 2014). This evidence suggests that stable E-Cadherin clusters are essential for junctional dynamics and morphological events. The balance between remodeling of adhesion via E-Cadherin clustering and transmission of force may facilitate mechanosensing and transduction at the junction (Fig 1.7) (Cavey et al., 2008; Guillot and Lecuit, 2013). 


\subsubsection{Interplay between cell adhesion and actomyosin dependent contractility}

E-Cadherin, together with other junction-associated proteins, for an adhesion complex. The intracellular domain of E-Cadherin binds to $\beta$-catenin and $\alpha$-catenin, forming a link to the underlying local actin-cortex. $\beta$-Catenin binds to the intracellular domain of E-Cadherin and forms a stable link. $\alpha$-catenin is known to dynamically bind to $\beta$-catenin and is essential for AJ formation and function. $\alpha$-catenin acts as an interface between cadherin-catenin complex and the actin cytoskeleton and can undergo a force-induced conformational change (Harris and Tepass, 2010)(Niessen et al., 2011). This makes $\alpha$-catenin a promising candidate as a force sensor. Conformational change in a-catenin eventually lead to the exposure of a binding site for another key actin-binding protein Vinculin (Fig 1.8, A) (Yonemura et al., 2010; Twiss et al., 2012). Recruitment of Vinculin to the AJ play a crucial role in anchoring junctions to the actin-networks. However, it has been shown that $\alpha$-catenin can also directly bind to F-actin (Drees et al., 2005) (Gates and Peifer, 2005). It is possible that rather being only a stable link to the actin-networks, $\alpha$-catenin instead acts as a key regulator of actin dynamics.

Once the forces are sensed through the adhesion complexes they are subsequently transmitted and non-muscle Myo-II motors assemble into antiparallel oligomers called minifilaments. These minifilaments processively move along the F-actin. In addition to regulating Myo-II dynamics, mechanical forces can also affect the activity of proteins involved in F-actin assembly and disassembly (BERSHADSKY et al., 2006) (Chanet and Martin, 2014).
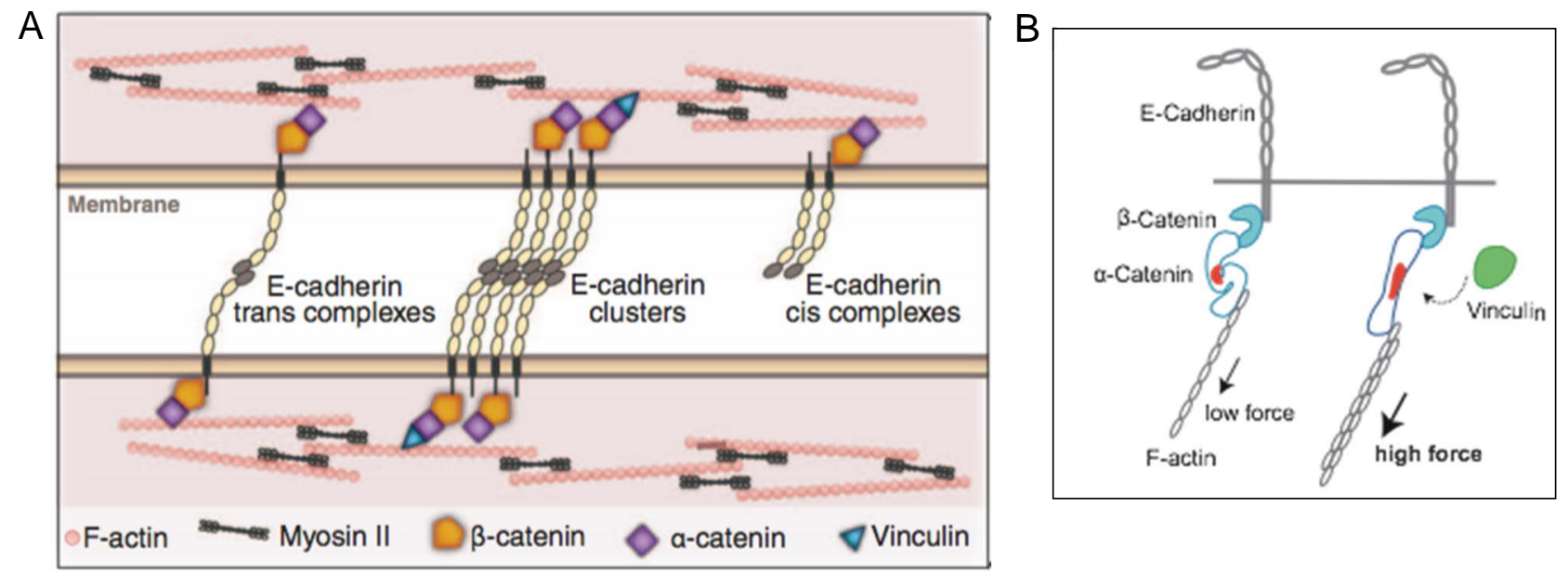

Fig 1.8 Adhesion complex at the epithelial junction. (A) E-cadherin forms cis and trans clusters stabilized by the interaction between extracellular domains and actin filaments via $\beta$-catenin, $\alpha$-catenin, and vinculin. (B) Schematic representation of $\alpha$-catenin undergoing conformation change in the presence of the force which eventually recruits Vinculin. Figure adapted from (Guillot and Lecuit, 2013) and (Kong et al., 2019). 


\subsubsection{Mechano-sensitive ion channels}

Behaving as molecular switches, mechano-sensitive ion channels transduce mechanical forces exerted on the cell membrane into electrical or biochemical signals. Molecular interactions between the ion channel and the plasma membrane are crucial for the force transduction. Since the actomyosin networks are linked to the plasma membrane through adhesion sites, changes in plasma membrane could render the information about the changes in intrinsic (generated by cortical actin networks) and extrinsic forces (via neighboring cells) (Diz-Muñoz et al., 2013). MS ion channels are able to sense the stretch or mechanical stimuli on the plasma membrane and can be activated by increased membrane tension. Once activated, MS ion channels can undergo conformational changes.

Mechanical gating or opening of the MS ion channels could be explained via two models discussed below (Fig 1.9):

(1) Membrane model in which the channel is sensitive to forces parallel to the plasma membrane (ex: Piezo)

(2) Tethered model, in which a channel interacts with accessory proteins (such as the cytoskeleton or cytoskeleton-regulating proteins) and force applied to the tether leads to changes in gating (ex:NompC) (Fig 1.11, B) (Christensen and Corey, 2007; Zhang et al., 2015).

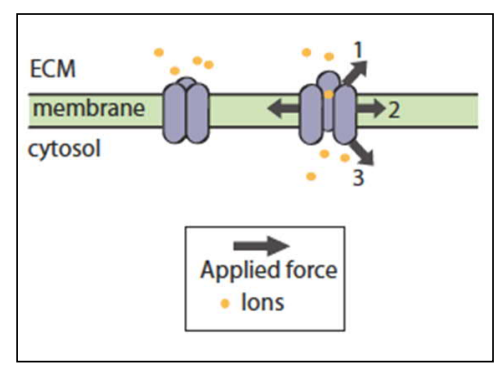

Fig 1.9 Model of MGC response to mechanical stimuli. The opening of MGCs allows ion flux, in response to the forces generated in (1) the extracellular space, (2) the plasma membrane, and/or (3) the cytoskeleton. Figure adapted from (Hunter et al., 2014).

The opening of MS channels can induce a range of cell responses by mediating the flux of specific ion. A very well studied example of MS ion channels is, signaling via $\mathrm{Ca}^{2+}$ dependent stretch-sensitive, TRP ion channels (transient receptor potential channel family) which result in actin cytoskeleton remodeling (Clark et al., 2008; Árnadóttir and Chalfie, 2010). In Drosophila photoreceptor cells, it has been shown that upon exposure to light, TRP channels 
can actively redistribute and reshape the actin cytoskeleton (Kosloff et al., 2003). In Drosophila, during DC, disrupting expression of dTRPA1 $\left(\mathrm{Ca}^{2+}\right.$ permeable TRP channel subunit) leads to severe closure defects. Perturbing ion channels by delivering toxic peptide GsMTx4 (alters the sensitivity of ion channels) to the embryos, shows a significant decrease in apico-medial actin networks. This Finding indicates that MS ion channels can affect actin dependent cell contractility (Hunter et al., 2014).

MS ion channels have been studied extensively so far in specialized mechanosensory neurons and largely in the context of sensory neurobiology, such as noci or auditory sensation (Lin and Corey, 2005; Kamikouchi et al., 2009; Effertz et al., 2011; Coste et al., 2012; Guo et al., 2016). Although the role of these ion channels in sensory-structures in adult flies has now been established, their function in epithelial cells during early embryonic development remained elusive. MS ion channels are widely expressed in non-neuronal tissue, as reported by genome-wide expression studies. Thus, MS ion channels might provide an alternative mechanism for force sensing. However, there has not been any detailed study dissecting the role of MS ion channels in the epithelial system.

\subsection{Role of MS ion channels in mechano-transduction across different species}

During this study, I have focused on understanding and exploring the role of MS ion channels in establishing coordinated cell-behavior via force sensing and mechano-transduction. Specifically, I investigated their function in the amnioserosa, a dynamic extra-embryonic epithelial tissue during early DC stage in Drosophila embryo (refer to section 1.3.2). To start with three candidate mechanosensitive ion channels namely putative MS ion-channel TMC, NOMPC, and Piezo, were chosen based on the knowledge and understanding about their role in sensory mechano-transduction in Drosophila (as discussed in the following sections) (Effertz et al., 2011; Kim et al., 2012; Zhang et al., 2015; Guo et al., 2016).

\subsubsection{Transmembrane channel-like protein (TMC)}

The TMC gene family were discovered around two decades ago through positional cloning of genes involved in hereditary deafness. Mutation in TMC1 (one of the members of the TMC gene family), causes dominant and recessive hearing loss in humans and is required 
for the auditory mechanical transduction by cochlear hair cells. The mouse ortholog for TMC1 also causes hearing loss. Mutations of TMC1 can alter the conductance and $\mathrm{Ca}^{2+}$ selectivity of the MET channels (cation channel with high selectivity for $\mathrm{Ca}^{2+}$ ) (Kurima et al., 2002; 2003; Kim and Fettiplace, 2013). TMC1 has multiple transmembrane topologies (10 transmembrane domains), resembling an ion channel or a functional subunit of an ion channel. Recently, mTMC1 has been predicted to be a pore-forming component of sensory transduction channels in auditory and vestibular hair cells (Fig 1.10, A) (Pan et al., 2018). Various studies exploring contributions of TMC1 to sensory function in hair cells provide genetic and physiological evidence supporting the role of TMC in mechano-transduction, yet the underlying molecular mechanism remains unclear (Pan et al., 2013; Fettiplace, 2016).

\section{Insight from sensory hair cells:}

The hair cells of the inner ear contain 'the hair bundle', which are a cluster of actin rich stereocilia located at the apical surface of the hair cells. Deflections in hair bundles in the presence of mechanical stimuli such as sound and pressure are converted into electrical signals via mechano-transduction. Extracellular protein filaments known as tip links are required for mechano-transduction and are thought to transmit physical force to the MS channels located at the stereocilia tips (Hudspeth, 1989). Tip links display heterophilic interactions with Protocadherin-15 (PCDH15) homodimers at the lower end and it has been shown that TMC interacts with transmembrane and cytoplasmic domains of PCDH15 (Fig 1.10, B) (Maeda et al., 2014; Beurg et al., 2015; Kurima et al., 2015).

A

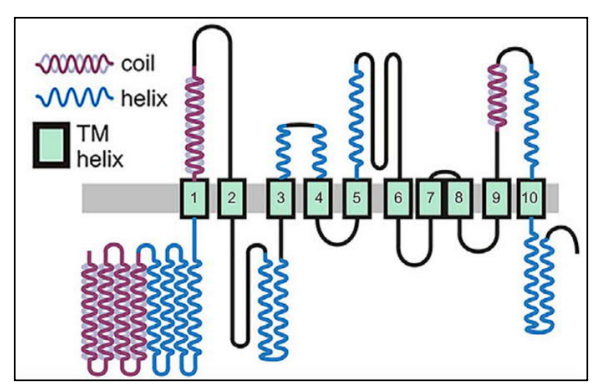

$\mathrm{B}$

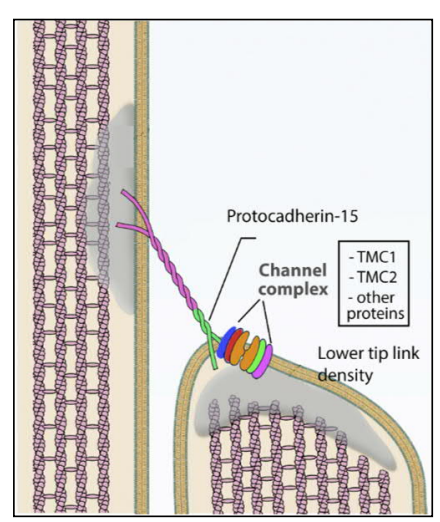

Fig 1.10 Transmembrane channel-like protein. (A) Predicted transmembrane topology for mmTMC1. (B) Schematic diagram of the stereocilia in inner hair cells. TMC1 and TMC2 localize with the components of the MET ion channel complex. Figure adapted from (Pan et al., 2018) and (Kurima et al., 2015).

TMC homologs are also found in other vertebrates (Gallus gallus, Xenopus laevis, Danio rerio) and invertebrates (Drosophila melanogaster, Caenorhabditis elegans). Only one tmc 
gene (CG46121) is found in Drosophila and the protein sequence is highly conserved with the TMC family members in other species. In Drosophila, in the sensory system, TMC is expressed in Class I da, Class II da, and bd sensory neurons and contributes to the mechanosensation in the body-wall. TMC mutant larvae display locomotion defects (Guo et al., 2016). Furthermore, food texture sensing in the adult fly labellum also depends on TMC, as it is expressed in the cluster of mechanosensory neurons at the fly tongue (Zhang et al., 2016). There has been no significant study regarding the role of TMC in the epithelium and if they are involved in the junctional mechano-transduction at the epithelial cell interface remains an open question.

\subsubsection{NOMPC (no mechanoreceptor potential C)}

NOMPC belongs to the TRP (Transient Receptor Potential) superfamily of cation channels (Clapham et al., 2001). In Drosophila, loss of function mutations in nompC gene affect mechanosensory responses and impair normal hearing, touch sensation, and have severe locomotion defects in the larvae and adult (Walker et al., 2000; Göpfert et al., 2006; Kamikouchi et al., 2009; Yan et al., 2013). NOMPC has 29 Ankyrin repeats (largest number of ARs among TRP channels). It is thought to be gated via tethering mechanism where ankyrin repeat domain tethers to the microtubule/ cytoskeleton and are able to displace and thereby activate the channel (Fig 1.11, B). Structurally ankyrin repeat domain of NOMPC resembles a helical spring which links mechanical displacement of the microtubule/cytoskeleton to the opening of the channel (Fig 1.11, A) (Zhang et al., 2015)(Jin et al., 2017).

Another example of $\mathrm{Ca}^{2+}$ permeable TRP channel subunit is Drosophila TRPA1 (Hamada et al., 2008). Knockdown of $d T R P A 1$ causes severe morphogenetic defects during $\mathrm{DC}$ and eventually leads to the failure of DC in the embryos (Hunter et al., 2014). Taking these findings into account suggests a crucial function of $\mathrm{Ca}^{2+}$ dependent ion channels, which further suggests that NOMPC may have an important role in establishing mechanotransduction at the epithelial interface. 

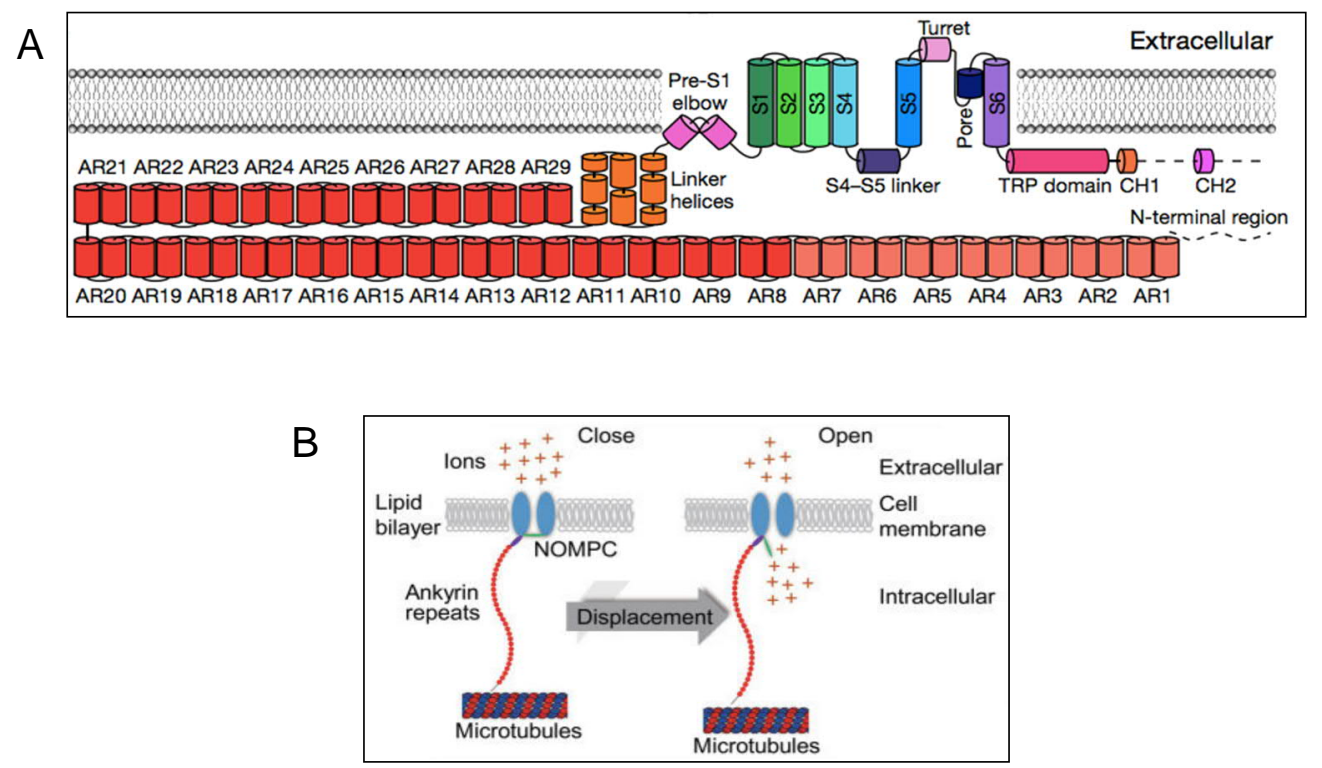

Fig 1.11 Mechanotransduction via NOMPC. (A) Schematic diagram illustrates major structural domains of one NOMPC subunit with long Ankyrin repeats. (B) Displacement of Ankyrin repeats tethered to the plasma membrane-associated microtubules can lead to channel activation. Figure adapted from (Jin et al., 2017) and (Zhang et al., 2015).

\subsubsection{Mechanosensitive Piezo channels}

Piezo family of MS ion channels are evolutionarily conserved transmembrane proteins. In mice, Piezo1 and Piezo2 are mechano-gated and can be modulated in the presence of mechanical stimuli. They are expressed in many mechanosensitive cell types for example, Piezo1 is expressed in endothelial cells of developing blood vessels in the mice. Piezo1 deficient embryos have defects in vascular remodeling, causing death mid-gestation (Ranade et al., 2014). Piezo2 deficiency in sensory neurons in mice as well as in humans results in loss of normal touch sensation and proprioception (Mahmud et al., 2017). Recent studies reveal a function of Piezo channel also in epithelial sheet homeostasis in MDCK cells. Upon experimental stretching of the epithelial sheet, Piezo1 channel is able to sense the mechanical stretch and activate which rapidly stimulates cell division (Gudipaty et al., 2017).

High-resolution cryo-electron microscopy structure of the mouse Piezo1 suggests that they organize at the membrane as a homotrimeric complex. This complex adopts a three-blade propeller shape (with at least 26 transmembrane helices per protomer) and an extracellular 
cap domain (Fig 1.12). The three propeller blades are predicted to be flexible and can adopt distinct conformations, with the help of a series of four transmembrane helix bundles termed as 'Piezo Repeats'. The structural information suggests that the membrane tension is able to induce conformational changes of the Piezo repeats which is transmitted via the beam and anchor domains to the inner helix and C-terminal domain, in turn activating the channel (Fig 1.12) (Saotome et al., 2018).

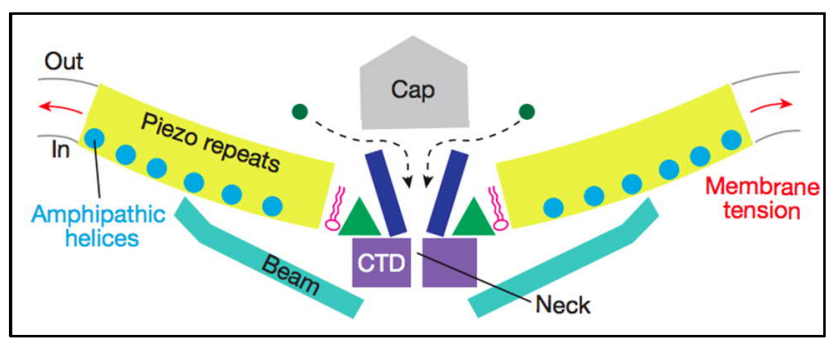

Fig 1.12 Structure and conformation of Piezo1. The scheme depicts the various structural elements of Piezo1 that contribute to its function as a mechanosensitive mechano-gated gated ion channel. Figure adapted from (Saotome et al., 2018).

In Drosophila there are two Piezo genes, Dmpiezo an ortholog, and piezo-like (pzl) a homolog of piezo gene families. Piezo deficiency in flies does not seem to have major developmental defects, as Piezo-KO flies are viable and fertile. However, noxious mechanical stimuli induced behavioral responses are severely reduced in Dmpiezo knockout larvae suggesting the role of Piezo in mechanosensory nociception (Kim et al., 2012). Loss of $p z l$ shows severe defects in crawling pattern and body gesture control in the larvae and can be rescued by expressing human or mouse Piezo1 (Hu et al., 2019). Interestingly, one of the recent studies suggests stretch-activated Piezo channel dependent, mechanical regulation of stem-cell differentiation in the adult fly midgut. Also, loss of piezo phenotypes in the midgut can be rescued by manipulation of cytosolic $\mathrm{Ca}^{2+}$ levels, suggesting that Piezo might function via $\mathrm{Ca}^{2+}$ signaling (He et al., 2018).

\subsection{Calcium signaling in epithelial morphogenesis}

Calcium signaling is known to have an impact in many developmental and morphogenetic events. In the epithelial cells, Calcium signaling is crucial for cytoskeletal dynamics. However, it is unclear how calcium is linked to actomyosin dependent contractile cell behavior. In cultured epithelial cells (human carcinoma line C-4II) there are evidences indicating the requirement of extracellular Calcium influx to induce epithelial cell contractions 
(Lee and Auersperg, 1980). In Xenopus, intracellular $\mathrm{Ca}^{2+}$ are required for neural tube formation. Transient increase in $\mathrm{Ca}^{2+}$ concentration induces apical constriction in the cells of the developing neural plate (Suzuki et al., 2017).

Ubiquitous involvement of $\mathrm{Ca}^{2+}$ signaling in multiple cellular functions is crucial during development. However, an excessive increase in $\mathrm{Ca}^{2+}$ also leads to cytotoxicity and cause cell death. The intracellular $\mathrm{Ca}^{2+}$ sensing mechanisms play a crucial role in the maintenance of $\mathrm{Ca}^{2+}$ homeostasis and signaling during cellular functions. Within the cytoplasm, under resting conditions, the $\mathrm{Ca}^{2+}$ concentration is maintained at $\sim 10^{-7} \mathrm{M}$ which is $10^{4}$ times lower compared to the $\mathrm{Ca}^{2+}$ concentration in extracellular spaces $\left(\sim 10^{-3} \mathrm{M}\right)$. Among other intracellular organelles, the endoplasmic reticulum (ER) can accumulate and maintain a higher $\mathrm{Ca}^{2+}$ than the cytoplasm $\left(1.5 \times 10^{-4} \mathrm{M}\right)$ as they are the main internal $\mathrm{Ca}^{2+}$ stores (Bagur, R. and Hajnóczky, 2017).

The increase in the intracellular $\mathrm{Ca}^{2+}$ concentration can be promoted by various stimuli such as extracellular signaling molecules, intracellular messenger, or mechanical stimulus, inducing an increase of $\mathrm{Ca}^{2+}$ concentration from $100 \mathrm{nM}$ to up to $1 \mu \mathrm{M}$ or more. This increase mainly occurs either via the influx of extracellular $\mathrm{Ca}^{2+}$ through $\mathrm{Ca}^{2+}$ dependent ion channels at the plasma membrane, or the release of $\mathrm{Ca}^{2+}$ from internal stores (from ER, via the 1,4,5triphosphate receptor (IP3R) and ryanodine receptor (RyR)). Synchronized activation and rapid deactivation of the ion channels leads to repetitive spikes or oscillation of intracellular $\mathrm{Ca}^{2+}$. The increase in intracellular $\mathrm{Ca}^{2+}$ is controlled via multiple positive and negative feedback mechanisms, maintaining homeostasis (Bagur, R. and Hajnóczky, 2017).

\subsubsection{Calcium signaling in Drosophila development}

In Drosophila, calcium signaling is involved in the regulation of multiple developmental stages starting from oogenesis to embryogenesis (Markova et al., 2019). As the Drosophila egg is ovulated, an intracellular calcium wave is observed to be spread across the oocyte. Interestingly, external pressure on the oocyte (or swelling by the oocyte) can also induce a similar rise in intracellular calcium flux presumably through the action of MS ion channels (Kaneuchi et al., 2015). During Drosophila oogenesis it has been shown that $\mathrm{Ca}^{2+}$ activated, Myosin II dependent contractions are able to generate active forces in order to control egg chamber elongation (He et al., 2010). 
Fig 1.13 Calcium signaling dependent cell contractions. (A) lon channels desensitized by toxic peptide GsMTx4 affects actomyosin dependent contractility. (B) $\mathrm{Ca}^{2+}$ dependent Myosin II activation in smooth muscle contraction Figure modified from (Hunter et al., 2014).

Cardiac muscle contractions during the larval stages depend on the Calcium-Induced Calcium Release (CICR) where transient calcium signals are able to promote its own release from intracellular calcium stores (from the sarcoplasmic reticulum). CICR release occurs via a specialized ion channel known as the ryanodine receptor (RyR) in cardiac muscle. The opening of the RyR is induced by the $\mathrm{Ca}^{2+}$ influx via calcium-dependent ion channel (for example TRP ion channel) and lead to the release of a larger amount of calcium from the SR. The RyR mutation leads to slow heartbeat in the larvae and has a role in heart function (Sullivan et al., 2000).

In germ-band epithelium $\mathrm{Ca}^{2+}$ uncaging induced, sudden $\mathrm{Ca}^{2+}$ bursts trigger cell contractions (Kong et al., 2019). These findings suggest that actomyosin cytoskeleton can be actively reorganized in a Calcium-dependent manner to drive contractility (Fig 1.12, A). An increase in intracellular free $\mathrm{Ca}^{2+}$ by photolytic uncaging as well as pharmacologic inhibition of $\mathrm{Ca}^{2+}$ channels using GsMTx4 leads to significant decrease in actomyosin network (Fig 1.13, A) (Hunter et al., 2014). During smooth muscle contraction, it is known that myosin II activation is regulated by $\mathrm{Ca}^{2+}$ calmodulin complex that activates myosin light chain (MLC) via MLC kinase (Fig 1.13, B) (Kitazawa et al., 1991). Recent studies in the epithelium, have shown that intracellular $\mathrm{Ca}^{2+}$ dependent non-muscle myosin II activation could be mediated via Rho kinase activity (Rok) (Kong et al., 2019). Yet the detailed mechanism of how $\mathrm{Ca}^{2+}$ can regulate actomyosin networks remained elusive so far in the non-muscle cells. 


\subsubsection{Calcium-dependent response to the epithelial wounding}

Calcium signaling plays a very significant role in wound healing. It is well understood that epithelial wound healing requires coordination of a group of cells to detect the presence of a wound and to migrate and proliferate in order to drive closure of the wounded site (AbreuBlanco et al., 2012; Enyedi and Niethammer, 2015). A very intriguing and crucial early aspect of the wound response is a rapid rise in cytosolic calcium within seconds of wounding. Studies across many organisms have shown that following the wound induction there is a rise in cytosolic calcium levels within the wounded cell which also spreads to the neighboring cells (Sammak et al., 1997; Antunes et al., 2013; Shannon et al., 2017). What initiates such a dynamic response across the field of cells is not very well understood. There are studies suggesting a change in tissue tension mediated opening of stretch-activated calcium ion channel or MS channel dependent model, which would allow propagation of $\mathrm{Ca}^{2+}$ signal across neighboring cells (Wood, 2012). In support of this hypothesis, in Drosophila wing disks, it has been shown that calcium waves can be generated by applying mechanical pressure which facilitates the opening of mechano-gated or MS ion channels (Restrepo and Basler, 2016). A detailed understanding of the role of MS ion channels during coordinated tissue-scale morphogenetic movement, in association with the cytoskeletal network, is required in order to fully understand the mechanism behind these complex events. Several factors, including the size and shape of the wound, and the intrinsic distribution of tension in the tissue are responsible to induce a multicellular coordinated mechanism in order to either repair or remove the wounded cell from the epithelial sheet. In the adult tissue, a commonly observed mechanism is crawling of lamellipodia at the leading edge of the wounded site in order to drive the wound closure.

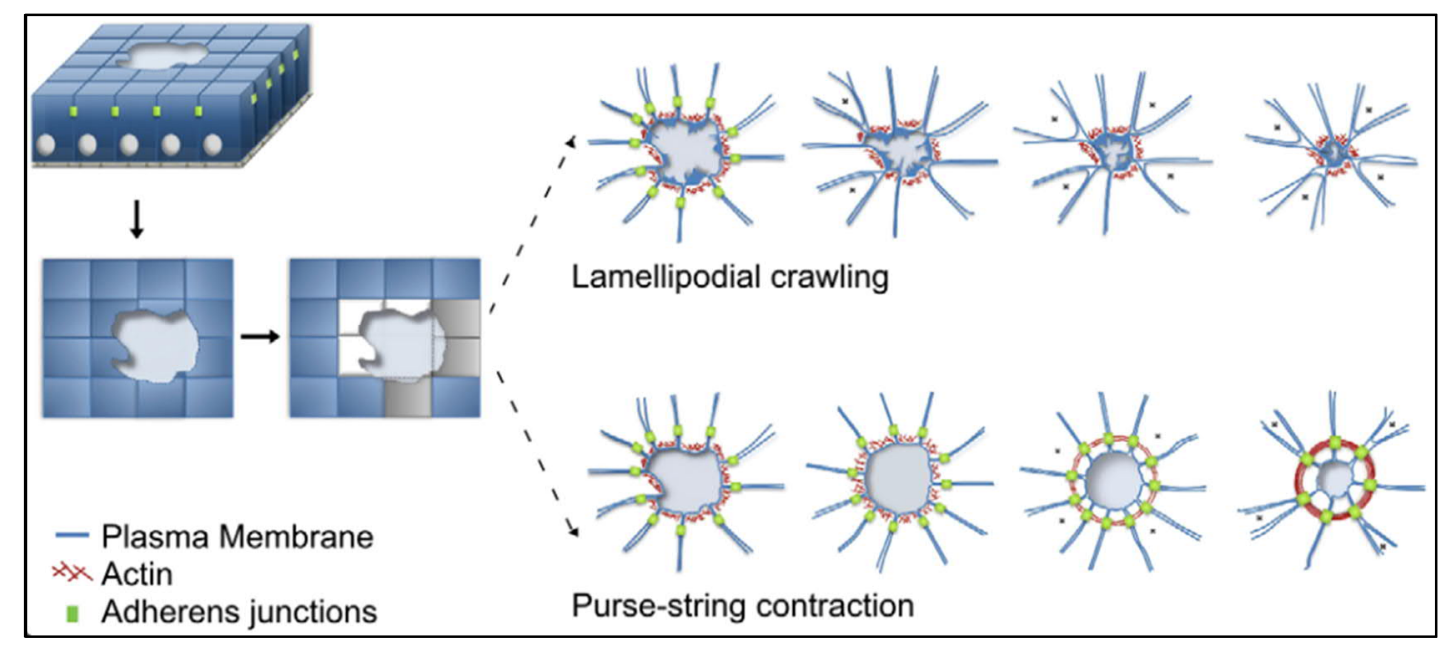


Fig 1.14 wound repair in Drosophila epithelium. The scheme shows phases of multicellular wound repair in epithelial cells. Damaged cells in the epithelial sheet are either repaired (gray) or removed (white) depending on the size and shape of the wound. Wound closure can then proceed with the help of dynamic actin and junctional remodeling and either via lamellipodial crawling, cells at the leading edge of the wound push themselves forward over the wounded site, or by the formation of an actin purse string which induces contractions to achieve wound closure. Figure adapted from (Abreu-Blanco et al., 2012)

The epithelial wound closure in the embryos is often mediated by the formation of an actomyosin purse string at the leading edge of the wounded site inducing contractile forces in order to achieve wound closure. The actin purse string is intercellularly linked by the adherens junctions (Fig 1.14) (Abreu-Blanco et al., 2012). Multiple actin regulators contribute to the assembly and remodeling of actin-rich protrusions at the wounded site. Studies in the ventral epidermis of stage 15 embryos suggest that the formation of actin protrusions is dependent on the activity SCAR, which is an activator of the Arp2/3 complex (branched actin remodeling protein complex). Loss of SCAR activity leads to complete loss of protrusion activity at the actin cable of the leading edge. However, the absence of protrusion activity only contributes to the speed of wound closure and wound healing still progresses at a relatively slow speed (Matsubayashi et al., 2015).

Wounded sites in the epithelium are known to have a highly dynamic activity of non-muscle myosin II, along with junctional (E-cadherin) remodeling (Fig 1.14) (Abreu-Blanco et al., 2012). As epithelial wounding is known to trigger $\mathrm{Ca}^{2+}$ influx, it may induce and regulate the contractile forces at the actin cable/purse string and drive the repair mechanisms (Paul Martin and Julian Lewis, 1992)

\subsection{Objective}

This study attempts to elucidate the role of putative mechanosensitive ion channel protein TMC (Transmembrane channel-like protein) in coordinated cell behavior and morphogenesis in the amnioserosa epithelium of Drosophila. Although having a substantial amount of information regarding their function and mechanism (as shown in the tip-links of the auditory hair cells), TMC has remained unexplored in the epithelial cells so far. We hypothesize that TMC is an essential component for coordinated and force-induced cell behavior in the AS. In epithelial cells, TMC may function in a $\mathrm{Ca}^{2+}$ dependent manner where it is able to transduce mechanical forces across neighbors via a molecular link with the adherens junctions. 


\section{Chapter 2. Material and methods}

\subsection{Material}

\subsubsection{General buffers}

\section{Table 2.1: List of general buffers}

\begin{tabular}{|l|l|}
\hline Agent & Ingredients \\
PBS (1X) & $\begin{array}{l}137 \mathrm{mM} \mathrm{NaCl}, 10 \mathrm{mM} \text { Na2HPO4, 2.7 mM KCL, 1.8 mM } \\
\text { KH2PO4, [pH 7.4], 1I } \\
0.25 \mathrm{M} \text { Tris, 2 M glycine, 1\% SDS, 1I }\end{array}$ \\
$\begin{array}{l}\text { SDS-Page buffer } \\
(10 \mathrm{x})\end{array}$ & $2 \mathrm{M}$ Tris, 50 mM EDTA, 1 M acetic acid [pH 8.5], 1I \\
\hline TAE-buffer (50x) & 193 mM glycine, 25 mM Tris, 5\% methanol, 1I \\
\hline Transfer-buffer (1x)
\end{tabular}

(For detailed list of Buffers, refer to section 2.1.12)

\subsubsection{Chemical reagents}

Chemicals used for this study were purchased from these companies unless otherwise stated:

- AppliChem GmbH (Darmstadt, Germany),

- $\quad$ Carl ROTH (Karlsruhe, Germany)

- Sigma-Aldrich (Steinheim, Germany).

\subsubsection{Enzymes and Kits}

Restriction enzymes used in this study were purchased from

- Fermentas/Thermo Fisher Scientific (Waltham, USA)

- New England Biolabs GmbH (Ipswich, USA). 
Table 2.2: List of enzymes from other sources.

\begin{tabular}{|l|l|}
\hline DNasel & Roche (Basel, Swiss) \\
\hline Long PCR Enzyme Mix & Fermentas \\
\hline Lysozym & AppliChem (Darmstadt, Germany) \\
\hline M-MLV Reverse transcriptase & Promega (Madison, USA) \\
PfuS mix DNA Polymerase & expressed and purified in the Großhans lab \\
\hline Proteinase K & Roche \\
\hline RQ1 RNase-free DNase & Promega \\
\hline T4 DNA Ligase & Fermentas \\
\hline T4 DNA Polymerase & Fermentas \\
Taq DNA Polymerase & expressed and purified in the Großhans lab \\
\hline
\end{tabular}

Kits were used as per the instruction manual from the manufacturer.

- Arcturus $^{\mathrm{TM}}$ PicoPure ${ }^{\mathrm{TM}}$ RNA isolation Kit, Thermo Fisher Scientific

- MiniElute Gel Extraction Kit, Qiagen (Hilden, Germany)

- Plasmid Midi Kit Nucleobond Xtra, Macherey-Nagel (Düren, Germany)

- PureLink ${ }^{\mathrm{R}}$ PCR-Purification Kit, Invitrogen (Carlsbad, USA)

\subsubsection{Antibodies}

Table 2.3 Primary antibodies used in the study.

\begin{tabular}{|l|l|l|l|} 
Antibody & Raised in & $\begin{array}{l}\text { Dilution and working } \\
\text { concentration }\end{array}$ & Source \\
\hline a-Tubulin & Mouse & $1: 50000(0.7 \mu \mathrm{g} / \mathrm{ml})($ Western $)$ & $\begin{array}{l}\text { Hybridoma bank } \\
\text { B512 }\end{array}$ \\
\hline Dlg & Mouse & $1: 100(\sim 0.4 \mu \mathrm{g} / \mathrm{ml})$ & $\begin{array}{l}\text { Hybridoma bank } \\
\text { 4F3 }\end{array}$ \\
\hline
\end{tabular}




\begin{tabular}{|l|l|l|l|}
\hline TMC & Rabbit & $1: 5000$ & Guo et al., 2016 \\
\hline NompC & Rabbit & $1: 1000$ & $\begin{array}{l}\text { (Liang et al., } \\
2011)\end{array}$ \\
\hline $\begin{array}{l}\text { E-Cadherin } \\
\text { (DCAD1) }\end{array}$ & Rat & $1: 100$ & $\begin{array}{l}\text { T. Uemura } \\
\text { (Oda et al., } \\
\text { 1993) }\end{array}$ \\
\hline
\end{tabular}

- Alexa-conjugated secondary antibodies were added at a final concentration of 1:500 (4 $\mu \mathrm{g} / \mathrm{ml}$ ) (Source: Life Technologies Carlsbad, USA).

- Phalloidin-Alexa 488: used for actin staining, in a final concentration of $6 \mathrm{nM}$ (Molecular Probes)

- GFP-Booster-Atto488 was added at a final concentration of $2 \mu \mathrm{g} / \mathrm{ml}$ (Source: Chromotek Martinsried, Germany).

- DNA staining was performed using DAPI. (Working concentration - 1:250 (0.2 mg/ml), Source: Sigma-Aldrich, St. Louis, USA).

- For western blots, secondary antibodies (IRDye-800CW and IRDye-680), were added at a dilution of 1:20000 $(0.05 \mu \mathrm{g} / \mathrm{ml}$ ) (Source: LI-COR Biotechnology Bad Homburg, Germany).

\subsubsection{Fly stocks}

Table 2.4 Fly stocks used in the study.

\begin{tabular}{|l|l|l|}
\hline Name & Genotype & Source \\
\hline OrR & $+/+$ & - \\
\hline E-Cad-GFP (K-in) & $\begin{array}{l}\text { w; DE-Cadherin-GFP[k-in]; } \\
+/+;+/+\end{array}$ & (Huang et al., 2009) \\
\hline myr-GCaMP6s & $\begin{array}{l}\text { W [1118];+/+; UASt- } \\
\text { GCaMP6s-myr; +/+ }\end{array}$ & (Chen et al., 2013) \\
\hline
\end{tabular}




\begin{tabular}{|c|c|c|}
\hline Utrophin-GFP & w; sqh-Utr::GFP/CyO & $\begin{array}{l}\text { Prof. T. Leciut (Levayer et } \\
\text { al., 2011) }\end{array}$ \\
\hline Mat 67,15 & $\begin{array}{l}\text { w; tub-Gal4-VP16 }\{w+\}[67] \\
\text { tub-Gal4-VP16 }\{w+\}[15] ;+++\end{array}$ & St. Johnston / Cambridge \\
\hline $\begin{array}{l}\text { Gla/CyO, twi>GAL4,UAS- } \\
\text { GFP }\end{array}$ & $\begin{array}{l}\text { w[1118]; In(2LR)Gla, wg[Gla- } \\
\text { 1]/CyO, P }\{w[+m C]=G A L 4- \\
\text { twi.G }\} 2.2, P\{w[+m C]=U A S- \\
\text { 2xEGFP }\} \text { AH2.2 }\end{array}$ & BDSC \\
\hline Ovo 2L & $\begin{array}{l}\text { y w hs-Flp [122]; ovoD Frt2L } \\
{[40 \mathrm{~A}] / \text { Cyo, hs-hid }\{w+\} ;+/+;} \\
+/+\end{array}$ & S. Luschnig / Münster \\
\hline 20X UAS-6X GFP & $\begin{array}{l}\text { y[1] w[]; PBac\{y[+mDint2] } \\
\text { w[+mC]=20XUAS- } \\
\text { 6XGFP\}VK00018/CyO, } \\
\text { P\{Wee-P.ph0\}Bacc[Wee- } \\
\text { P20] }\end{array}$ & BDSC \\
\hline$T M C^{\Delta}$ & $\mathrm{w}[*] ;+/+; t m c^{G a l 4}$ & $\begin{array}{l}\text { Guo et al., } 2016 \\
\text { (Martin Göpfert/Dept. of } \\
\text { Cellular } \\
\text { Neurobiology,Göttingen) }\end{array}$ \\
\hline Tmc-Gal4 & $\mathrm{P}\{$ Tmc-GAL4.3.0\} & Zhang et al., 2016 \\
\hline xit & $\begin{array}{l}\text { w xit[X-330] f Frt }[9-2,18 E] \\
\text { Flp122\{ry+\} /FM7c, y w[a] sn } \\
\text { B; +/+; +/+ }\end{array}$ & $\begin{array}{l}\text { Germline clone screening } \\
\text { (Vogt et al., 2006) }\end{array}$ \\
\hline xit; E-Cad-GFP & $\begin{array}{l}\text { w xit[X-330] f Frt [9-2, 18E] } \\
\text { Flp122\{ry+\}/FM7c; DE- } \\
\text { cadherin-GFP[k-in]; +/+ }\end{array}$ & $\begin{array}{l}\text { D. Kong / } \\
\text { J. Grosshans lab }\end{array}$ \\
\hline nompC-Gal4 & $P\{$ nompC-GAL4.L\} & Liu et al., 2007 \\
\hline nompC RNAi & $\begin{array}{l}\mathrm{y}[1] \mathrm{v}[1] ; \mathrm{P}\{\mathrm{y}[+\mathrm{t} 7.7] \\
\mathrm{v}[+\mathrm{t} 1.8]=T R i P . J F 01067\} \text { attP2 }\end{array}$ & BDSC \\
\hline nompC Trojan-gal4 & $\begin{array}{l}\text { W[*]; nompC } C_{\text {Trojan-gal4 } / C y O,} \\
\text { P\{Wee-P.ph0\}Bacc[Wee- } \\
\text { P20]; +/TM6b }\end{array}$ & $\begin{array}{l}\text { Martin Göpfert/Dept. of } \\
\text { Cellular } \\
\text { Neurobiology,Göttingen }\end{array}$ \\
\hline
\end{tabular}




\begin{tabular}{|c|c|c|}
\hline $\begin{array}{l}\text { nomp } C^{\text {Trojan-gal4; }} \\
\text { nomp } C^{3}, \text { nompc GFP }\end{array}$ & $\begin{array}{l}\text { w["]; nomp } C^{\text {Trojan-gal4 }} \\
\text { /nompC } C^{3} \text {, UAS-nompC::GFP }\end{array}$ & $\begin{array}{l}\text { Martin Göpfert/Dept. of } \\
\text { Cellular } \\
\text { Neurobiology,Göttingen }\end{array}$ \\
\hline Piezo [KO] & $\begin{array}{l}\text { w[^]; } \\
\text { PBac }\{w[+m C]=R B 5 . W H 5\} P i e \\
\text { zo }[K O]\end{array}$ & BDSC \\
\hline Piezo-Gal4 & $\begin{array}{l}\left.\mathrm{w}{ }^{*}\right] ; \mathrm{P}\{\mathrm{w}[+\mathrm{mC}]=\text { Piezo- } \\
\text { GAL4.1.0\}III }\end{array}$ & Chadha and Cook, 2015 \\
\hline P\{UAS-Piezo.GFP $\} \| A-2$ & $\begin{array}{l}\left.\mathrm{w}{ }^{*}\right] ; \mathrm{P}\{\mathrm{w}[+\mathrm{mC}]=\mathrm{UAS}- \\
\text { Piezo.GFP }\} \| \mathrm{IA}-2 / \mathrm{CyO} ; \\
\text { MKRS/TM6B, Tb[1] }\end{array}$ & BDSC \\
\hline Piezo Mi\{MIC $\}$ gfp & $\begin{array}{l}\text { y[1] w[67c23]; Mi\{PT- } \\
\text { GFSTF.0\}Piezo[MI04189- } \\
\text { GFSTF.0] }\end{array}$ & BDSC \\
\hline
\end{tabular}

Table 2.5 Fly stocks generated in the study.

\begin{tabular}{|c|c|}
\hline Name & Genotype \\
\hline E-Cad-GFP; $T M C^{\Delta}$ & w; DE-Cadherin-GFP[k-in]; $t m c^{\text {Gal/4}} / T M 3$ \\
\hline$T M C^{\Delta}$;myr-GCaMP6s & w; +/+; UASt-GCaMP6s-myr, TMC ${ }^{\Delta}$ \\
\hline Utrophin-GFP; $T M C^{\Delta}$ & w;UASp-GFP-Utrophin; $t m c^{\text {Gal/ }}$ \\
\hline Tmc::eGFP & W;;CRISPR Tmc::eGFP(K-in) (InDroso) \\
\hline xit;; GCaMP6s-myr & $\begin{array}{l}\text { w xit[X-330] f Frt [9-2, 18E] Flp122\{ry+\}/FM7c;+/+; UASt- } \\
\text { GCaMP6s-myr }\end{array}$ \\
\hline E-Cad-GFP, nompC $C^{\text {Trojan-gal4 }}$ & w; E-Cad-GFP, nompC Trojan-gal4, Frt (neo) 2L/CyO; +/+ \\
\hline nompC (CRISPR) KO & $\begin{array}{l}\text { w; nompC } C^{K O}:: \text { attP::loxP-3xP3-Ds-Red::loxP/Cyo; +/+ } \\
\text { (InDroso) }\end{array}$ \\
\hline
\end{tabular}




\subsubsection{Fly media}

\section{Fly food}

$64 \mathrm{~g}$ of agar was cooked and homogeneously dissolved in seven liters of water. To this 0.64 $\mathrm{g}$ maize meal, $80 \mathrm{~g}$ soya-bean meal, $200 \mathrm{~g}$ of baker`s yeast, $640 \mathrm{~g}$ malt extract and $175 \mathrm{~g}$ sugar-beet molasses were added. Food was properly mixed and cooked for another $30 \mathrm{~min}$. After the food was cooled down to $55-60^{\circ} \mathrm{C}, 12 \mathrm{~g}$ Nipagin (dissolved in $90 \mathrm{ml}$ ethanol) and $75 \mathrm{ml}$ propionic acid was mixed into the food. Food was then filled into the vials and kept overnight to cool down and solidify at room temperature. Vials were plugged with sponge plugs the next day. Ready to use fly food vials were stored at $18^{\circ} \mathrm{C}$.

\section{Apple juice agar plates}

To prepare one liter of apple juice agar, $17.5 \mathrm{~g}$ agar was dissolved in $750 \mathrm{ml}$ of water. The mixture was autoclaved and post-autoclaving a mix of $25 \mathrm{~g}$ sugar was dissolved in $250 \mathrm{ml}$ of apple juice. To the agar solution, $1.5 \mathrm{~g}$ of Nipagin (dissolved in $10 \mathrm{ml}$ of ethanol) was added. After the mixture was allowed to cooled down to $60^{\circ} \mathrm{C}$. Apple juice agar was poured to the Petri dishes and plates were stored at $4^{\circ} \mathrm{C}$.

\subsubsection{Media for bacterial culture}

\section{LB-medium}

$10 \mathrm{~g}$ Bactotryptone, $5 \mathrm{~g}$ Yeast extract, and $10 \mathrm{~g}$ Sodium chloride were dissolved homogeneously in water, in order to make one liter of LB-medium. Media was then autoclaved and stored at $4^{\circ} \mathrm{C}$.

\section{LB-plates}

To make LB-plates, $10 \mathrm{~g}$ agar and the desired antibiotic was added after LB-solution was cooled down to $55^{\circ} \mathrm{C}$. LB-solution was then poured into Petri dishes and the dishes were stored at $4{ }^{\circ} \mathrm{C}$. 


\subsubsection{High Resolution Microscopy}

Spinning Disk microscope:

Cameras:

Optics:

Lasers:

Objectives:

Laser ablation system:

Lasers:

Filter:

Confocal Microscope

LSM 780:

Detection:
Zeiss Observer.Z1, CSU-X1 (Carl Zeiss, Jena, Germany, 2009)

AxioCam MRm

AxioCam ICc1

emCCD evolve 512

Epifluorescence, DIC

488nm (20mW, OPSL)

$561 \mathrm{~nm}(20 \mathrm{~mW}, \mathrm{DPSS})$

A. Plan (10X, NA 0.25)

Plan-Apochromat (25X, NA 0.5, multiimmersion, DIC)

Plan-Neofluar (40X, NA 1.3, oil, DIC)

LCIPlan-Neofluar (63X, NA 1.3, multiimmersion, DIC)

Plan-Apochromat (100X, NA 1.4, oil)

UGA-42 (Rapp OptoElectronic, Wedel, Germany)

DPSL-355/14: $355 \mathrm{~nm}$ pulsed YAG laser, $14 \mathrm{~mW}$ average power, $70 \mu \mathrm{J}$ per pulse, Up to $200 \mathrm{~Hz}$

TB355/405/473

Zeiss AxioObserver.Z1

(Carl Zeiss Jena,Germany, 2011)

PMT

GASP

cooled PMT 
Lasers:

Objectives:

\subsubsection{Equipment}

Other microscopes:

- Axioplan 2 Fluorescence Microscope, Zeiss

- MZ12.5, Leica

- PrimoVert Inverted Microscope, Zeiss

- Stemi 2000 Stereomicroscope, Zeiss

- SteREO Lumar V12, Zeiss

- SZX12 Fluorescence Stereo Microscope, Olympus

\section{Other equipment:}

- Centrifuges, Eppendorf

- ChemiDoc, Biorad

- Concentrator, Eppendorf
Diodelaser (405nm, 30mW)

Argonlaser $(458 \mathrm{~nm}, 488 \mathrm{~nm}, 514 \mathrm{~nm}$, $25 \mathrm{~mW}$ ) DPSS-Laser (561nm, 20mW) $\mathrm{HeNe}$-Laser (633nm, 5mW)

EC Plan-Neofluar (10x, NA 0.3)

LCI Plan-Neofluar (25x, NA 0.8, multiimmersion, DIC)

C-Apochromat (40x, NA 1.2, water)

Plan-Apochromat (63x, NA 1.4, oil)

LCI Plan-Neofluar (63x, NA 1.3, multiimmersion) 
- DynaMagnet, life technologies

- Electrophoresis Constant Power Supply, Pharmacia

- FemtoJet, Eppendorf

- High Pressure Homogenizer/Mickrofluidizer- EmulsiFlex-C5, Avestin

- NanoDrop 2000c, Thermo Scientific

- Odyssey CLx Infrared imaging system, LI-COR Biosciences

- Real time PCR machine - rotor gene 6000, Corbett/Quiagen

- Thermomixer, Eppendorf

- Trans-Blot Semidry, Biorad

- Ultrasonics Sonifier-450, Branson

\subsubsection{Other reagents and materials}

- Agarose, Invitrogen

- Aquapolymount, Polysciences, Inc.

- BSA/Albumin Fraction V, Roth

- Cover Slides, Thermo Scientific

- CitraSolve (Danbury, Connecticut)

- DanKlorix

- DNA ladder (1 kb), Thermo Scientific

- dNTPs, Thermo Scientific

- Falcon tubes (15 ml, $50 \mathrm{ml}$ ), BD Falcon 
- Filter papers (Ø110 mm) Macherey-Nagel

- Fly cages (Ø 50 mm, Ø 94 mm)

- Fly vials, Greiner

- Forceps

- Formaldehyde (37\%), Sigma-Aldrich

- Glass homogenizer, B. Braun Biotech International

- Glass pipettes (5 ml - $25 \mathrm{ml})$, Silber Brandt

- Glass slides, Thermo Scientific

- Green Taq buffer (10x), Thermo Scientific

- Halocarbon oil 700, Sigma-Aldrich

- Immersol 518F/DROSOPHILA, Zeiss

- Mifepristone (RU468), Sigma-Aldrich

- Milk powder, Sucofin

- Parafilm M, Bemis

- Pasteur pipettes, Brandt

- Petri dishes, Greiner

- Phenol/Chloroform/lsopropanol, Roth

- Pipetman $(2 \mu \mathrm{l}, 10 \mu \mathrm{l}, 200 \mu \mathrm{l}, 1000 \mu \mathrm{l})$, Gilson

- Pipette tips, Sarstedt

- Platinum wire 
- Poly-L-Lysine Solution (0.1\%), Sigma-Aldrich

- Polypropylene column, Quiagen

- Pre-stained Protein Ladder, Thermo Scientific

- Protease Inhibitor Cocktail - Complete Mini (EDTA free), Roche

- Proteinase K, Roche

- Protran Nitrocellulose Membrane, Amersham

- Sodium Hypochlorite solution (15\%), Sigma-Aldrich

- TRI-reagent, Sigma-Aldrich

- TritonX-100, Roth

- Tween20, Roth

- Viva spin, Sartorius

- VoltaLef Oil 10s, Lehmann, Voss \& Co.

- Weigh Boat, VWR

- Whatman $3 \mathrm{~mm}$ blotting paper, GE healthcare

- Yeast, Dr. Oetker 


\subsubsection{Software}

- Adobe Illustrator CS6, Adobe

- Adobe Photoshop CS6, Adobe

- GraphPad Prism6, GraphPad Software, Inc.

- FIJI (NIH)

- Image Studio, LI-COR Biosciences

- Microsoft Excel 2013, Microsoft

- Microsoft Word 2013, Microsoft

- NanoDrop 2000, Thermo Fischer Scientific

- SeqBuilder, DNASTAR

- Zen 2012, Carl Zeiss

\subsubsection{Buffers}

Buffers preparation was done according to Sambrook and Russel, 2001 unless otherwise stated.

1) Buffers for DNA extraction from adult flies:

Homogenization buffer

$10 \mathrm{mM}$ Tris/HCl pH7,5

$60 \mathrm{mM} \mathrm{NaCl}$

10 mM EDTA 
2) Buffer for Immuno-staining and western blot:

PBS

$130 \mathrm{mM} \mathrm{NaCl}$

$7 \mathrm{mM} \mathrm{Na} 2 \mathrm{HPO} 4$

$3 \mathrm{mM} \mathrm{NaH} 2 \mathrm{PO} 4$

$\mathrm{pH} 7.4$

PBST

$0.1 \%$ Tween 20 in PBS

Embryo fixation solution

$4.5 \mathrm{ml} 1 \mathrm{X}$ PBS

0.5 or $1 \mathrm{ml}$ Formaldehyde (37\%)

$5 \mathrm{ml}$ Heptane

Immuno-staining blocking buffer

$5 \%$ BSA in $1 \times$ PBS

Western blot blocking buffer

$5 \%$ milk powder in $1 \mathrm{X}$ PBS

3) Buffer for Miniprep of plasmid DNA:

Solution I

$50 \mathrm{mM}$ Tris/ $\mathrm{HCl}, \mathrm{pH} 8.0$

10 mM EDTA

Solution II

$1 \%$ SDS

$0.2 \mathrm{M} \mathrm{NaOH}$

Solution III

3 M Potassium acetate

Adjusted to $\mathrm{pH} 5.4$ with acetic acid

4) Buffer for Insitu Hybridisation:

NTP+Dig labelling mix (10x):

Dig-11-UTP: $10 \mathrm{mM}$ solution (25

$\mu l$, Roche \#1209256)

$10 \mathrm{mM}$ ATP

10 mM GTP 
$10 \mathrm{mM}$ CTP

6,5 mM UTP

3,5 mM Dig-11-UTP, pH7,5

transcription buffer

for SP6, T3, T7 RNA

polymerase, store at $-20^{\circ} \mathrm{C}$

400 mM Tris- $\mathrm{HCl}, \mathrm{pH} 860$ mM

$\mathrm{MgCl} 2$

100 mM DTT

20 mM Spermidine

$100 \mathrm{mM} \mathrm{NaCl}$

AP buffer

$100 \mathrm{mM} \mathrm{NaCl}$

$50 \mathrm{mM} \mathrm{MgCl} 2$

100 mM Tris pH9.5 0,2\% Tween

$\mathrm{ddH}_{2} \mathrm{O}$

$1 \mathrm{ml}(5 \mathrm{M}) 2,5 \mathrm{ml}(1 \mathrm{M}) 5 \mathrm{ml}(1$ M) $500 \mu \mathrm{l}(10 \%)$ ad $50 \mathrm{ml}$

NBT/BCIP

NBT (nitrobluetetrazolium, Sigma)

$75 \mathrm{mg} / \mathrm{ml}$ in $70 \%$ DMF

BCIP (X-phosphate, Sigma)

$50 \mathrm{mg} / \mathrm{ml}$ in DM 
store at $-20^{\circ} \mathrm{C}$

5) Buffer for immunoprecipitation:

RIPA buffer

$10 \mathrm{mM}$ Tris/HCl pH 7.5

$150 \mathrm{mM} \mathrm{NaCl}$

$0.1 \%$ SDS

1\% TritonX 100

$1 \%$ Deoxycholate

5 mM EDTA

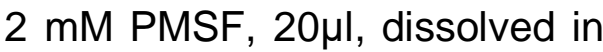
isopropanol (freshly added)

1X Roche protease inhibitor cocktail (freshly added)

6) Buffer for ion channel blocking $25 \mathrm{mM} \mathrm{MgCl}_{2}$

$10 \mathrm{mM} \mathrm{NaCl}$

$2 \mathrm{mM} \mathrm{KCl}$

2mM EGTA

$5 \mathrm{mM}$ Hepes

$25 \mathrm{mM} \mathrm{MgCl} 2$

$\mathrm{pH} 7.5$

All buffers were filtered before use 


\subsection{Methods}

\subsubsection{Genomic DNA extraction from adult flies}

Approximately 200 adult flies were taken and anesthetized. Flies were transferred to a mortar filled with liquid nitrogen and were ground with a pestle until a homogenous mixture was formed. Dounce homogenizer was precooled on ice. This mixture was transferred to a pre-cooled Dounce homogenizer containing $5 \mathrm{ml}$ of homogenization buffer. The mixture was centrifuged at $1000 \mathrm{rpm}$ for a minute in order to remove the debris. The supernatant was carefully taken out and transferred to a new Eppendorf tube and centrifuged at $8000 \mathrm{rpm}$ for $5 \mathrm{~min}$ in order to separate the nuclei. The pellet was resuspended in $0.5 \mathrm{ml}$ homogenization buffer. The sample was incubated at $37^{\circ} \mathrm{C}$ for 45 to $60 \mathrm{~min}$. After incubation, $50 \mu \mathrm{l}$ of $10 \%$ SDS and proteinase $\mathrm{K}$ (at the final concentration of $100 \mu \mathrm{g} / \mathrm{ml}$ ) were added and mixed well.

$0.5 \mathrm{ml}$ phenol/chloroform was added to the sample and mixed for 5 minutes. The sample was centrifuged at $13,000 \mathrm{rpm}$ for $5 \mathrm{~min}$. To remove protein impurity completely, the upper phase was taken out and transferred to a new Eppendorf tube. This step was repeated twice. After adding and mixing $0.5 \mathrm{ml}$ of Chloroform to this the sample was centrifuged again at $13,000 \mathrm{rpm}$ for $5 \mathrm{~min}$. Once again, the upper phase was taken out and transferred to a fresh Eppendorf tube. $\mathrm{NaCl}$ was added to a final concentration of $200 \mathrm{mM}$ and mixed well. $100 \%$ ethanol (two volume) was added and mixed gently. The DNA was visible at the interface as a clump. The DNA pellet was precipitated by centrifugation of $13000 \mathrm{rpm}$ for $5 \mathrm{~min}$. The pellet was washed in $80 \%$ ethanol and then $100 \%$ ethanol. The supernatant was discarded and the pellet was dried in the Speedvac. The dried pellet was resuspended in $0.5 \mathrm{ml}$ of TE buffer.

\subsubsection{Polymerase chain reaction (PCR)}

PCR reactions were performed by using Taq - polymerase (for general reactions) or PfuSpolymerase (for reactions requiring improved proofreading). PCR reactions were set up with the following reagents:

1. $0.5-2.5 \mu l$ forward Primer $(10 \mu \mathrm{M})$

2. $0.5-2.5 \mu$ reverse Primer $(10 \mu \mathrm{M})$

3. $1.0 \mu \mathrm{ldNTP} \operatorname{mix}(10 \mathrm{mM})$ 
4. 1x Buffer (Green Taq Buffer (10x)/ Phusion HF buffer (5x))

5. $0.3 \mathrm{U} / \mu \mathrm{l}$ Polymerase (Taq/PfuS)

6. $50-200$ ng DNA template

7. $\operatorname{ad} 50 \mu \mathrm{lddH} 2 \mathrm{O}$

\section{PCR program for DNA amplification by Taq or PfuS polymerase:}

1. Initial Denaturation: $95^{\circ} \mathrm{C}, 2 \mathrm{~min} / 98,5^{\circ} \mathrm{C}, 30 \mathrm{~s}$

2. Denaturation: $95^{\circ} \mathrm{C}, 30 \mathrm{~s} / 98,5^{\circ} \mathrm{C}, 5-10 \mathrm{~s}$

3. Annealing: $X{ }^{\circ} \mathrm{C}$ (Oligonucleotide specific), 30 s $25-35 \mathrm{x}$

4. Extension: $72{ }^{\circ} \mathrm{C}, 1 \mathrm{~kb} / \mathrm{min} / 72{ }^{\circ} \mathrm{C}, 1 \mathrm{~kb} / 15-30 \mathrm{~s}$

5. Final Extension: $72{ }^{\circ} \mathrm{C}, 10 \mathrm{~min}$

\subsubsection{Phenol-Chloroform extraction}

A DNA/RNA sample was taken and volume was increased up to four-fold by adding ddH2O. $200 \mu \mathrm{l}$ of Phenol/Chloroform/Isopropanol (25:24:1) was added and mixed. The Sample was centrifuged for $5 \mathrm{~min}$ at 13,000 rpm and the upper phase was transferred to a new reaction tube. $200 \mu \mathrm{l}$ of chloroform was added, mixed well and centrifuged the same as the previous step. To precipitate the DNA was upper phase was transferred to a new reaction tube. 1/10 volume of sodium acetate $(3 \mathrm{M})$ and 2.5 volumes of ethanol $(100 \%)$ was mixed. The sample was stored at $-20^{\circ} \mathrm{C}$ for overnight or at $-80^{\circ} \mathrm{C}$ for at least $30 \mathrm{~min}$. The sample was then centrifuged at $14,000 \mathrm{rpm}$ for $30 \mathrm{~min}$ at $4^{\circ} \mathrm{C}$. The supernatant was removed and the pellet was washed with $200 \mu \mathrm{l}$ of ethanol $(70 \%)$. Centrifugation step was repeated and the supernatant was removed. DNA pellet was dissolved in 10-30 $\mu \mathrm{l}$ of ddH2O. 


\subsubsection{In situ hybridization}

Embryos were dechorionated using 50\% bleach and were fixed (as described in section 2.2.12) and stored at $-20^{\circ} \mathrm{C}$. Fixed embryos were transferred to PBST, rinsed $3 X$ in PBST (5min incubation) and 2X in 1:1 hybridization-solution(hyb-sol)/PBST (10 min). Embryos were incubated $10 \mathrm{~min}$ in hyb-sol. Embryos were then prehybridize in hyb-sol for $1 \mathrm{~h}$ at $57^{\circ} \mathrm{C}$. Finally, the RNA probe was added in hyb-sol and was incubated overnight at $57^{\circ} \mathrm{C}$ (RNA probe in hyb-sol can be saved to be reused). Next day the embryos were rinsed $3 x$ with prewarmed hyb-sol and washed $3 \times 30$ min in hyb-sol at $57^{\circ} \mathrm{C}$.

Embryos were washed in the following manner

- $10 \min 4: 1 \mathrm{hyb}-$ sol/PBST at $57^{\circ} \mathrm{C}$

- 10 min 3:2 hyb-sol/PBST at $57^{\circ} \mathrm{C}$

- $10 \min 2: 3$ hyb-sol/PBST at $57^{\circ} \mathrm{C}$

- $10 \min 1: 4$ hyb-sol/PBST at room temperature

- Embryos were washed 2X 20 min in PBST with 1\% BSA at room temperature and rinsed with PBST.

\section{Detection with AP}

Alkaline phosphatase coupled Digoxigenin antibody (Boehringer, Fab Fragments) was added at the dilution of 1:2000 in PBST. The embryos were incubated for 2 Drosophila at room temperature or overnight at $4^{\circ} \mathrm{C}$. Embryos were rinsed $3 \mathrm{X}$ with PBST and washed 4X15 min with PBST. Embryos were washed again 3X5 min with AP buffer.

To start the reaction, $4,5 \mu \mathrm{l} \mathrm{NBT}$ and 3,5 $\mu \mathrm{l} \mathrm{BCIP}$ was added into $1 \mathrm{ml}$ of AP buffer and incubated until color developed (NOMPC, TMC - overnight, Piezo - 4 hours). After incubation embryos were rinsed $4 \mathrm{X}$ with PBST. The embryos were washed with ethanol series up to $100 \%$ ethanol $(20 \%, 40 \%, 60 \%, 80 \%, 100 \%)$, and each step for $10 \mathrm{~min}$ in order to wash out the background. The embryos were washed again going down the ethanol series to only PBT and were mounted in aquapolymount. 


\subsubsection{Western blot}

Embryos staged from 12-24 hours, were collected on apple juice agar plates. Embryos were dechorionated in $50 \%$ Klorix bleach for $1 \mathrm{~min}$. The dechorionated embryos were collected into an Eppendorf tube and weighed. The weight of the embryos was determined ( $1 \mathrm{mg}$ $=100$ embryos). The embryos were snap frozen and stored in liquid nitrogen. The embryos were homogenized in 1X Lämmli buffer with the volume to make the final concentration 20 embryos $/ \mu$ l. The sample was heated to $95^{\circ} \mathrm{C}$ for $5 \min \left(65^{\circ} \mathrm{C}\right.$ for $\left.\mathrm{TMC}\right)$ and followed by centrifugation at $14,000 \mathrm{rpm}$ for $1 \mathrm{~min}$.

After centrifugation, the supernatant (protein extracts) was loaded on the SDS-PAGE. Using a semi-dry transfer, the proteins from the gel were transferred onto a nitrocellulose membrane (1 hour at $60 \mathrm{~V} / \mathrm{gel}$ ). The membrane was blocked in $5 \%$ milk powder in freshly made PBST for at least $30 \mathrm{~min}$ and incubated with primary antibody either overnight at $4^{\circ} \mathrm{C}$ or 2 hours at the room temperature. The membrane was rinsed $3 X$ with PBT followed by 4X15 min PBST washes. The membrane was incubated with secondary antibody for 1 hour at room temperature, covered and protected from light. The membrane was rinsed in PBST for 3X followed by 4X15 min PBST washes. Odyssey CLx Infrared Imaging system was used to detect the bands.

\subsubsection{Co-Immunoprecipitation (Co-IP) with Tmc::eGFP and sepharose beads GFP binder}

Tmc::eGFP expressing embryos collected on apple-juice agar plates, were staged from 1020 hours. Embryos were dechorionated in 50\% Klorix bleach for $1 \mathrm{~min}$. The dechorionated embryos were collected into an Eppendorf tube and weighed. The weight of the embryos was determined ( $1 \mathrm{mg}=100$ embryos). Embryos were stored liquid nitrogen.

Sepharose-GFP-binder beads (Chromotek) were vortexed and $20 \mu$ l was pipetted into $500 \mu l$ cold RIPA buffer. Then it was centrifuged for 2 mins and $8000 \mathrm{rpm}$ at $4^{\circ} \mathrm{C}$. The supernatant was discarded and the centrifugation step was repeated twice as above.

The embryo was transferred to pre-cooled homogenizer filled with $600 \mu$ of RIPA buffer, homogenized. Homogenized embryos were transferred to a pre-cooled Eppendorf and centrifuged for 15 mins at 14,000 rpm. Lipid layer was carefully removed and the supernatant was transferred to a new Eppendorf. After taking $10 \mu l+20 \mu l$ Lämmli input sample, the lysate was loaded on the beads and incubated overnight at $4^{\circ} \mathrm{C}$. 
After incubation, beads were centrifuged for $2 \mathrm{mins}$ and $8000 \mathrm{rpm}$ and unbound sample was taken out $(10 \mu \mathrm{l}+20 \mu \mathrm{l}$ Lämmli). Beads were washed 3 X2min in $1 \mathrm{ml}$ RIPA at $8000 \mathrm{rpm}$.

Bound proteins were eluted from the beads in $20 \mu \mathrm{l}$ Lämmli buffer. The sample was heated to $65^{\circ} \mathrm{C}$ (for transmembrane proteins) for $5 \mathrm{~min}$ and centrifuged at $14,000 \mathrm{rpm}$ for $1 \mathrm{~min}$. Samples (along with the beads) were loaded on SDS-PAGE and followed by western blot.

\subsubsection{Generation of germline clones}

The germline clones were generated following the protocol by Chou and Perrimon (Chou and Perrimon, 1992) (includes minor modifications). Flippase induction was mediated by the heat shock at $37^{\circ} \mathrm{C}$ for $60 \mathrm{~min}$ per day for two days (24-48 hour and $48-72$ hour) posthatching. For xit germline clones, duration of the heat shock was modified to once for 15 mins.

\subsubsection{UAS-GAL4 system}

The UAS-GAL4 system is used as a tool to ectopically over-express the gene of interest tissue as well as stage-specific manner (Brand and Perrimon, 1993). Binding of a yeast transcription factor GAL4 to an upstream activating sequence (UAS), allows the activation and expression of the target gene. In UAS reporter flies the target gene is placed under the control of UAS. In a GAL4 driver line, GAL4 gene is under the control of a promoter or enhancer of a gene with a known expression pattern.

\subsubsection{Meiosis recombination}

For Meiotic recombination, 'Frt2L2R/al dp b pr Frt2L, 2L367' virgins were collected and crossed with 'al dp b pr Bl c px sp/SM1' males. The heterozygotes for the chromosome carrying mutant (nompC (CRISPR) KO, nompC $C^{\text {Trojan-Gal4}}$ ) and the recessive markers and Frt2L2R recombined during meiosis. During this process various classes of recombinant chromosomes were segregated and deposited to the female egg which could be detected by crossing with 'al dp b pr Bl c px sp/SM1' males.

\subsubsection{Lethality test}


In order to estimate the total number of embryos which failed to develop to the larval stage lethality test was done. Flies were allowed to lay eggs for 0-5hrs at room temperature on apple juice agar plates. The embryos were collected in a sieve and washed. Embryos were then aligned on agar plates and kept at room temperature for further development. The number of unhatched embryos was counted after $24 \mathrm{hrs}$. and $48 \mathrm{hrs}$.

\subsubsection{1 larval cuticles preparation}

Embryos were collected overnight and incubated for another 24 hours and allowed to age and complete the embryonic development. The embryos were then washed by transferring into a sieve, dechorionated (using $50 \%$ Klorix for 2 mins) and washed several times with water. Using a fine brush, dechorionated embryos were then transferred to a clean slide into a drop of the mixture of Hoyer's medium (Hoyer, 1882) and lactic acid. The embryos were covered with a clean coverslip and incubated overnight at $65^{\circ} \mathrm{C}$. Cuticles were images using a dark field optic or phase contrast.

\subsubsection{Embryo fixation and Immuno-staining}

\section{Embryo fixation}

Embryos of desired stages were dechorionated using $50 \%$ of Klorix for 2 min. The embryos were collected in a sieve and washed thoroughly with water to remove any trace of Klorix completely. $5 \mathrm{ml}$ heptane, $4.5 \mathrm{ml} 1 \mathrm{x}$ PBS and $0.5 \mathrm{ml}$ formaldehyde (37\%) were added to a scintillation vial. Embryos were added to this scintillation vial and fixed for $20 \mathrm{~min}$ at room temperature under constant shaking. The lower phase containing formaldehyde was removed carefully. $5 \mathrm{ml}$ of methanol were added to the embryos and the embryos were vortexed for $30 \mathrm{~s}$ to remove the vitelline membrane. Devitalized embryos were transferred to an Eppendorf tube. The embryos were washed with methanol multiple times and were stored at $-20^{\circ} \mathrm{C}$.

\section{Hand devitalization of the early Drosophila embryos}

For Phalloidin and nompC staining, the embryos were hand devitalized. After 20 min fixation in $5 \mathrm{ml}$ heptane, $4.5 \mathrm{ml}$ 1XPBS and $0.5 \mathrm{ml}$ formaldehyde (37\%), embryos are removed from the interface of upper and lower phase and washed with 1x PBS. Embryos were placed on a double-sided tape attached to a petri dish lid. A drop of $0.1 \%$ PBST was added on the top 
of the embryo. The vitelline membrane was manually removed by hand devitalizing the embryos using 2-3gauge needles under a dissecting microscope. Devitalized embryos started to float into the PBST and thus, were collected.

\section{Embryo immuno-staining}

Fixed embryos stored in methanol or hand-devitalized were rinsed multiple times and washed once for $5 \mathrm{~min}$ with $1 \mathrm{ml} 1 \mathrm{X}$ PBST. Blocking step was performed by incubation of embryos for $1 \mathrm{~h}$ in $500 \mu \mathrm{l}$ PBT containing 5\% BSA at room temperature. Primary antibodies were diluted in the indicated ratio in $500 \mu$ of PBT with $1 \%$ BSA and incubated for 2 hours at room temperature or overnight at $4{ }^{\circ} \mathrm{C}$ (Table 2.4). Embryos were rinsed $3 \mathrm{X}$ and washed for $4 \mathrm{X}$, every 15 minutes with $500 \mu \mathrm{l}$ PBT. The secondary antibody was added and incubated for 1 hour at room temperature. Washing steps were performed using the same procedure mentioned above (all incubation step was done under continuous rotation). In the final washing step, embryos were stained with DAPI (1:250, $0.2 \mathrm{mg} / \mathrm{ml}$ ) for 2-5min, rinsed $3 X$ and washed once again for $5 \mathrm{~min}$ with PBT. Embryos were mounted in Aquapolymount medium.

\subsubsection{Permeabilization of vitelline membrane for Drug perturbation}

Embryos were dechorionated and washed with distilled water. Permeabilization by immersion in a 1:10 solution of CitraSolve and water, for 2mins (Rand et al., 2010). Embryos were washed multiple time with 1X PBS. Embryos were dried carefully (as they appear softer) and glued to the coverslip and submerged in PBS. 10mins before the live imaging, PBS was removed and the embryos were submerged in $5-50 \mathrm{mM} \mathrm{MgCl}_{2}$ solution in order to block all the Calcium-dependent ion channels (Hartzell and White, 1989).

\subsubsection{Live imaging of Drosophila embryo}

Stage 10-14 embryos were collected and dechorionated using $50 \%$ of Klorix for $2 \mathrm{~min}$. The embryos were transferred and collected in a sieve and washed multiple times with water to remove any traces of Klorix completely. Dechorionated embryos were lined with dorsal-up orientation (in order to image AS cells) on an agar block and were glued on to a coverslip. Embryos were covered in a drop of halocarbon oil before imaging. Time-lapse image stacks were recorded using spinning disc microscope with a Zeiss X40, N.A. 1.3, and water- 
immersion objective every 10 seconds at a single plane. Images were processed using ImageJ/Fiji.

\section{The time interval for data acquisition (Frame rate)}

- Recording for oscillatory cell behavior in AS - 10s/frame (40X), 35s/frame (25X)

- $\quad$ Laser cut and wounding experiments - 1s/frame

\subsubsection{Laser ablation and wounding}

Embryos were collected on apple juice agar plates at $25^{\circ} \mathrm{C}$ for 2 hours, and aged for 15 hours at $22^{\circ} \mathrm{C}$ (until early dorsal closure stage) and were dechorionated. Embryos were lined on agar block (in dorsal-up orientation) and transferred to a coverslip and dried for $2 \mathrm{~min}$, in a desiccation chamber. Before imaging embryos were covered in a drop of halocarbon oil. The UV laser (DPSL355/14, $355 \mathrm{~nm}, 70 \mu \mathrm{J} /$ pulse, Rapp Optoelectronic) was targeted to the cells from the epi-port of the microscope and was controlled by an independent scanning head (Rapp Optoelectronic). An ablation was targeted in a single cell, at the plane of adherens junction (or in the middle of the cell for wounding experiments) with the $1.5 \%$ of laser power. The exposure time was kept at 200 milliseconds (around 40 pulses) during recording mode on an inverted spinning disc microscope (Zeiss, 40X oil, $N$ 1.3) with a CCD camera (Zeiss). Images were recorded every $1 \mathrm{~s}$ and $0.3 \mathrm{~s}$ per frame (for different experiments). The image stacks were processed using ImageJ/Fiji.

The imaging microscope and laser ablation system were independently installed; therefore, an ablation was performed during imaging on an inverted spinning disc microscope. Laser ablation experiments for the recoil velocity measurement was carried out at the plane of adherens junction. Images were acquired every 0.3 s using spinning disc microscope (Zeiss, 40X oil, N 1.3). Imaging started approximately around $1 \mathrm{~min}$ prior to the ablation and was stopped at around $5 \mathrm{~min}$ after the ablation. Recoil velocity after the laser ablation was measured by tracking the displacement between two adjacent $3 \mathrm{X}$ vertices from the target junction. Initial recoil velocity was measured using the first five seconds of the displacement trajectory of the junctional recoil after the laser cut.

Cell wounding was done using a $355 \mathrm{~nm}$ pulsed YAG laser (DPSL-355/14, Rapp OptoElectronic) illumination. 'Click and Fire' mode was chosen, while a movie was recorded 
on a spinning disc microscope (Zeiss, 40X oil, N1.3). With 1.5\% laser power (around 0.5 $\mu \mathrm{J} /$ cell), the laser was exposed for 1500 milliseconds (around 300 pulses) per cell. Images were acquired every $0.3 \mathrm{~s}$ using spinning disc microscope (Zeiss, $40 \mathrm{X}$ oil, N 1.3).

\subsubsection{Large-scale Image segmentation}

Large-scale recordings in multiple embryos of all genotype was performed. The continuous cell shape change during AS oscillations were acquired. During this study, an improved image segmentation method was developed by our collaborators Matthias Häring (AG Fred Wolf, Dept. of Theoretical Neurophysics, Max-Planck-Institute for Experimental Medicine, Göttingen, Germany). New machine learning technique based on deep neural networks was applied as a segmentation approach of the AS (Ronneberger et al., 2015). Cycle-consistent generative adversarial networks (GAN) segmentation was used as our main approach for processing of our large-scale time-lapse data in this study. Reconstruction of cell shape dynamics for complete AS tissue was achieved using Cycle-GAN trained with manually created ground truth images. (Haering et al., 2018). 


\section{Chapter 3. Results}

The initial experiment to observe the expression of the ion channels in the amnioserosa cells using GFP reporter lines, expressed from the promoter of ion-channel proteins (tmc, nompC, and piezo, was performed by Dr. Deqing Kong and I repeated and reconfirmed them. Starting from this step, I carried the study further. I performed all the biological experiments, time-lapse recordings, laser cut experiments, wounding assays and Fiji based quantifications, shown in this study. The segmentation of AS cells via deep neural network and machine learning was performed by Matthias Häring (Dept. of Theoretical Neurophysics, Max-Planck-Institute for Experimental Medicine, Göttingen, Germany) (section 3.4). Large-scale computational analysis for spatiotemporal changes in the cellular anisotropy (section 3.5), AS cell oscillatory dynamics (section 3.6.1), and cell-pair coupling type analysis were done by Matthias Häring (section 3.4.2-3).

\subsection{Expression of mechanosensitive ion channels in the AS epithelium}

First of all, I analyzed the expression of the three candidate proteins, putative MS ion channel TMC, and MS ion channel NOMPC, and Piezo in the epithelial cells in Drosophila embryos. I used GFP reporter UAS-nls-GFP/UAS-palmitoylated-GFP, which were driven by the GAL4 expressed from the promoters of nompC, tmc and piezo (Zhang et al., 2016) (Fig. $3.1 \mathrm{~A})$.

\subsubsection{Expression of TMC, NOMPC, and Piezo in the epithelial cells during embryogenesis}

TMC was found to be expressed in the epidermis and the amnioserosa (AS) during dorsal closure (DC), in stage 13-14 embryos. The signal to noise ratio for tmc-Gal4 driven GFP reporter was high probably due to relatively low expression of TMC during early embryogenesis stages. TMC accumulated as clusters or puncta in the AS cells (Fig 3.1, B). nompC-Gal4 driven GFP reporter expression was observed in the AS, as well as in the epidermis (Fig 3.1, C). Interestingly, I found that the GFP reporter, expressed via piezoGAL4 driver, displayed mosaic expression in the AS tissue (Fig 3.1, D). However, this experiment did not display the actual localization of the ion channel proteins as in this case, 
the specific localization of the ion channel proteins inside the cells were dependent on the type of GFP reporter line used with the desired promotor Gal4 line.

A

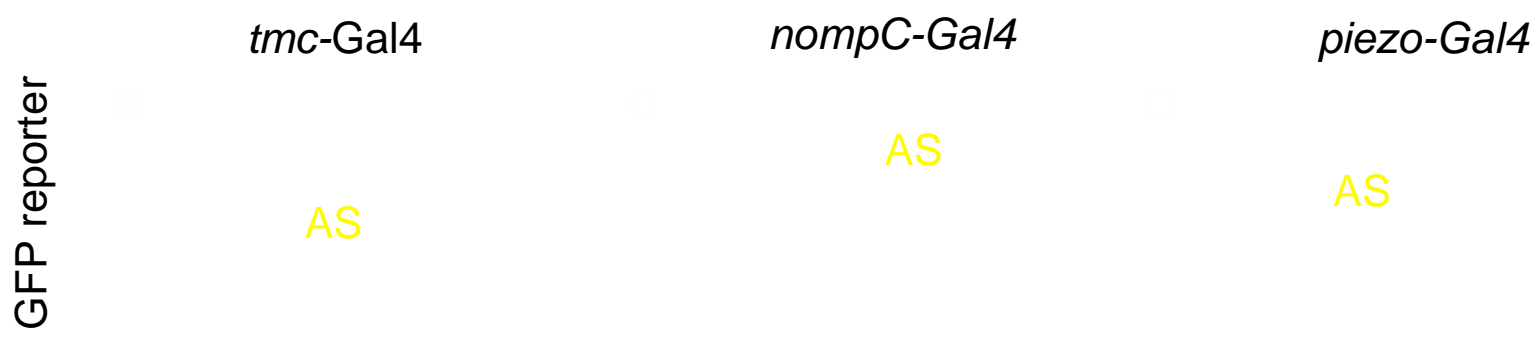

Fig 3.1 Expression of mechanosensitive ion channels in AS epithelium. (A) Scheme depicts GFP reporter genes, driven by GAL4 enhancer traps for the $t m c$, nompC, and piezo. (B) tmc-Gal4 was crossed with palmitoylated GFP reporter, (C) nompC and (D) piezo-Gal4 lines were crossed with nls-GFP reporter. The dotted line in yellow marks the boundary between AS and epidermis in the early DC stage embryos. Scale bar $=10 \mu \mathrm{m}$. (All Images are maximal intensity projections of multiple z-stacks).

\subsection{Sub-cellular localization of ion-channels in the AS cells}

In order to find out the specific sub-cellular localization of the endogenous TMC and NOMPC ion channel proteins, immuno-staining with polyclonal antibodies were performed. In the case of Piezo, sub-cellular localization was detected using a GFP-tagged fusion protein.

\subsubsection{TMC is uniformly expressed at the apical and apico-lateral membrane in the} AS cells

Immunostaining of AS cells in Dorsal closure stage embryos using TMC antibody suggested that TMC was uniformly expressed apically and at the membrane/cell border of AS cells (Fig $3.2 \mathrm{~B}, \mathrm{C})$. To check the specificity of the protein localization, I performed immunostainings in the $T M C^{\Delta}$ mutant embryos. $T M C^{\Delta}$ allele is a knock-in of GAL4, substituting transmembrane region of the TMC locus (Fig 3.2, A) (transgene provided by Prof. Martin Göpfert, Cellular Neurobiology, University of Göttingen). The staining for TMC was specific, since I detected strongly reduced signals in the $T M C^{\Delta}$ embryo. Also, the membrane localization of TMC was completely lost in the $T M C^{\Delta}$ mutant embryos (Fig 3.2, C). I also 
performed phalloidin staining in $T M C^{\Delta}$ mutant embryos which suggested that $\mathrm{F}$-actin levels in the $T M C^{\Delta}$ mutants were comparable to the wild-type.

A

C

Dapi

TMC

F-actin

B AJ

을

$\underbrace{\delta}_{i}$

Fig 3.2 Cellular localization of TMC in the AS cells. (A) TMC mutant allele: knock-in of GAL4 substituting transmembrane region of the TMC locus (Guo et al., 2016). (B) Scheme depicts the cellular distribution of TMC in AS cells. (Green=TMC localization, AJ=adherens junction) (C) Immunostaining of fixed embryos, with antibodies against DAPI (nuclear staining) TMC and F-actin (Phalloidin) in wild-type and $T M C^{\Delta}$ mutant embryos (surface and sagittal views). Scale bar=10 $\mu$ m.

\subsubsection{NOMPC localizes as clustered structures at the membrane in the AS cells}

Immunostaining with NOMPC antibody suggested that NOMPC was distributed as punctate/clustered structures at the apical and apico-lateral membrane of the AS cells (Fig 3.3). However, the expression and distribution of NOMPC was strongly reduced in nompC$R N A i$, expressed under control of Maternal-Gal4 driver. Also, the overall F-actin levels were 
lowered. Specifically, the apical actin protrusions in the cells seemed to be affected. This phenotype is yet to be reconfirmed in the NOMPC null mutants (NOMPC(CRISPR)KO) .
DAPI
NOMPC
F-actin
Merge

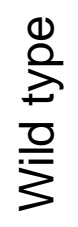

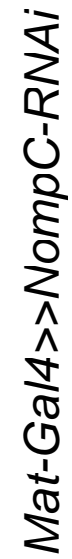

Fig 3.3 Cellular localization of NOMPC in the AS cells. Immuno-staining of fixed embryos with antibodies against DAPI (nuclear staining), NOMPC and F-actin (Phalloidin) in the wild-type and nompC-RNAi embryos (surface and sagittal). Scale bar=10 $\mu \mathrm{m}$. 


\subsubsection{Piezo localizes as clusters at the membrane and apico-medial region in the}

AS cells

In order to detect sub-cellular localization of Piezo I expressed UAS-Piezo-GFP driven via Amnioserosa-Gal4. The signal was detected as a distribution of punctate structures along the apical and lateral membrane (Fig. 3.4, A). I followed the dynamics of Piezo-GFP positive puncta in the oscillating AS cells, at a higher magnification (100X). Piezo puncta appeared to move dynamically on the apical/apico-medial surface, in a similar fashion as Myosin II dynamics previously shown in the AS cells (Fig. 3.4, C). The oscillation of Piezo puncta also coincided with the cell shape changes during AS dynamic oscillations however, phalloidin staining revealed that F-actin levels were not affected in the Piezo-KO embryos (Fig. 3.4, C).

A AS/Gal4>>UAS/Piezo/GFP
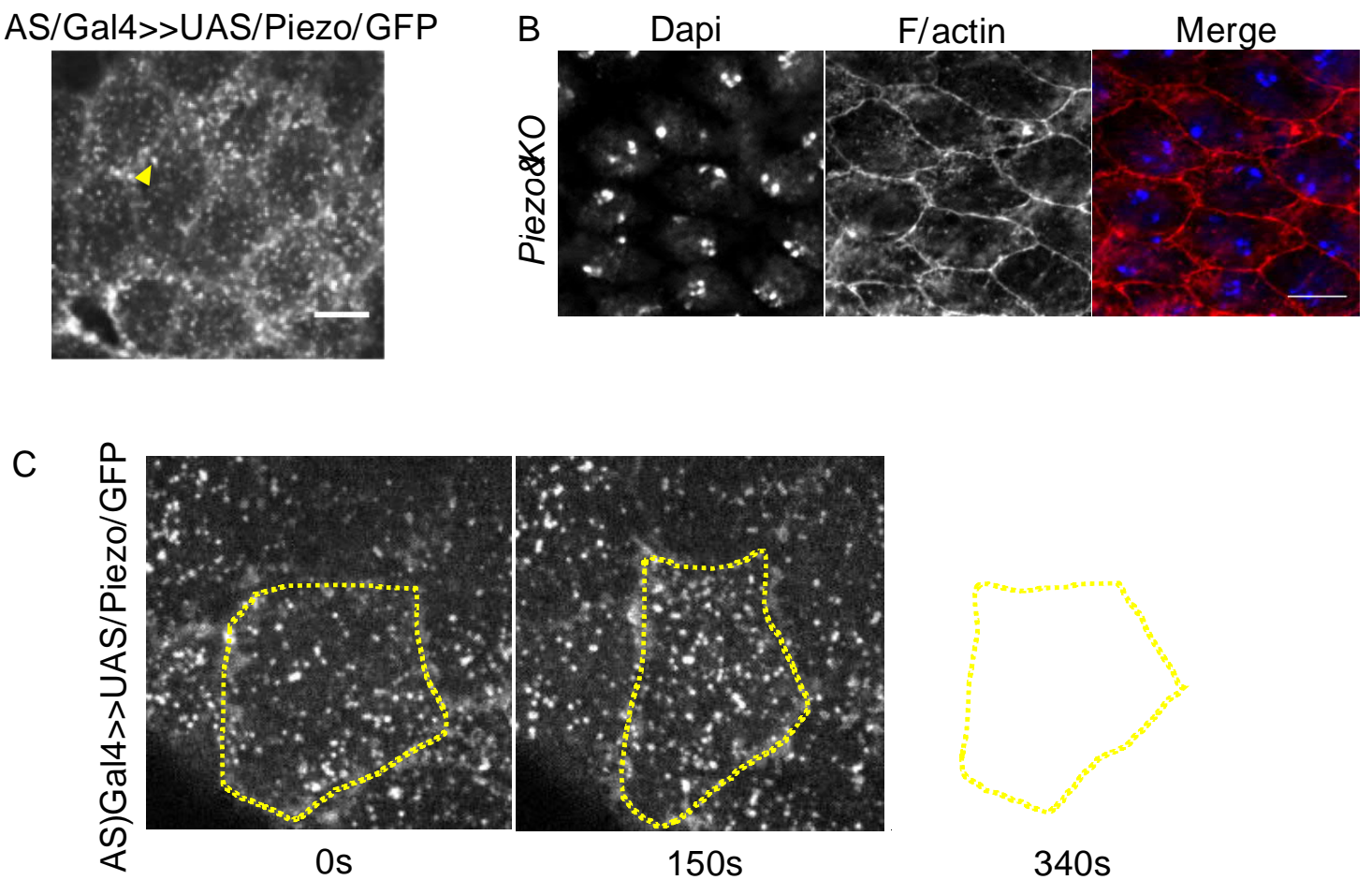

Fig 3.4 Cellular localization of Piezo in the AS cells. (A) UAS-Piezo-GFP expressed via tissue-specific ASGal4 driver. (B) Fixed Piezo-KO embryos stained for Phalloidin (F-actin). (C) Piezo GFP positive puncta accumulate as foci at the apico-medial region of the AS cells and oscillate over time. Dashed line indicates the cell outline. Scale bar $=10 \mu \mathrm{m}$. 


\subsection{TMC and NOMPC depletion lead to the embryonic lethality and cuticle defects in the embryo}

I analyzed TMC and NOMPC mutant embryos for hatching rate as well as for proper cuticle formation in unhatched embryos. $T M C^{\Delta}$ mutants showed a mild $18 \%$ of embryonic lethality. For NOMPC depletion in the embryos, nompC-RNAi was expressed maternally (via maternal-Gal4 driver) and zygotically (via AS-Gal4 driver), in the same embryo. Among the total population of observed embryos ( $\mathrm{N}=300), 50 \%$ of the embryos showed embryonic lethality. Piezo-KO embryos were viable and fertile with no embryonic lethality and cuticle defects.

Further, I analyzed extracellular cuticles from unhatched, $T M C^{\Delta}$ and nompC-RNAiembryos. Unhatched $T M C^{\Delta}$ mutant embryos showed a range of cuticle phenotypes, in which cuticle hole remained a dominant phenotype, and was found in $70 \%$ of the total population of unhatched embryos (Fig. 3.5, B).

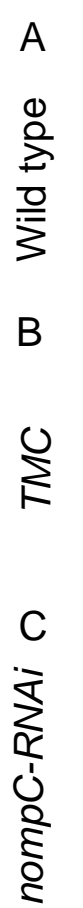

\section{Embryo 1}

Embryo 2

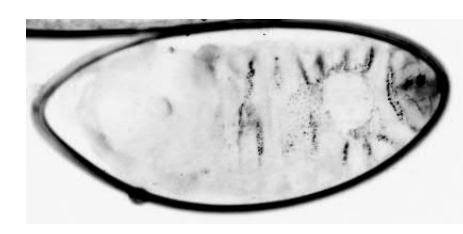

Embryo 3

Fig 3.5 Cuticle phenotype in (A) Wild-type (B) unhatched $T M C^{\Delta}$ and (C) nompC-RNAi embryos. (Yellow arrow marks the cuticle hole).

Approximately $50 \%$ of the unhatched embryos expressing nompC-RNAi, showed cuticle hole phenotype (among other cuticle phenotypes) thus, indicating perturbed epithelial integrity in NompC depleted embryos (Fig. 3.5, C). 


\subsection{Imaging and segmentation pipeline for large-scale computational image analysis}

To study the dynamic cell behavior and morphogenesis in an unbiased manner in the AS cells, large scale imaging, segmentation, and computational analysis were performed in multiple embryos. An optimized and robust large-scale imaging pipeline was established during this study. Embryos from all the genotypes, were prepared for live imaging under same conditions and at room temperature (for detail description refer to the section 2.1.4). Overnight collections of stage 10-14 embryos were done. Time-lapse recordings of the live wild-type, $T M C^{\Delta}$, and xit embryos were done in order to analyze multiple parameters of cell behavior (cell anisotropy, cell dynamics, cell-area) in the whole AS tissue. 25X objective was used for well-resolved view of the complete AS tissue. To specifically analyze detailed cellular and junctional morphology, high resolution images were acquired using $40 \mathrm{X}$ objective.

Oscillatory behavior of AS cells was followed over time in the E-cadherin labelled embryos. Multiple time frames (including z-stacks) were acquired starting from the end of germ-band retraction to the initiation of the DC (stage 13-14). I chose a time point based on the distance traveled by the germ-band relative to the cephalic furrow of the embryo, during the course of germband retraction. All the embryos from wild-type, $T M C^{\Delta}$ and xit were aligned to this time-point, referred to as the end of germ-band retraction stage. Images were acquired at the interval of $35 \mathrm{~s}$ per frame for $1.5 \mathrm{~h}$ in wild-type and $T M C^{\Delta}$, and for $3.5 \mathrm{~h}$ in $x$ it embryos (due to delayed progression of germ-band retraction to complete open amnioseros stage).

For further quantitative analysis, segmentation of the AS tissue was done. Segmentation approach was developed and performed by Matthias Häring. In order to quantitatively analyze spatiotemporal morphological behavior and cell dynamics of AS cells in detail, the whole AS tissue was reconstructed. Deep convolutional networks and a new network and training architecture called Cycle-GAN was used, to achieve a near complete reconstruction of the cell shape and dynamics for all the cells in the AS tissue (Haering et al., 2018). (Fig 3.6 A, B). Machine learning based image processing techniques, used for automated segmentation and image processing applications, typically require very large and manually prepared, ground truth segmentation training datasets. 
A

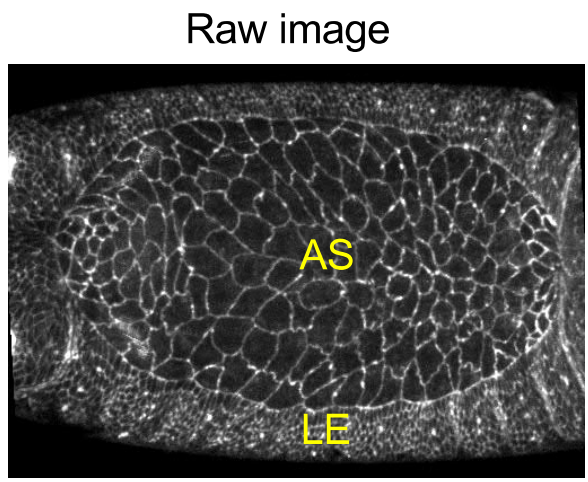

B
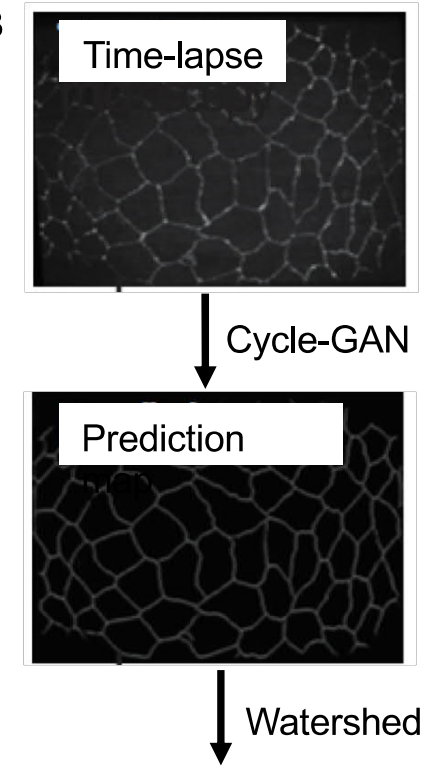

Segmentation
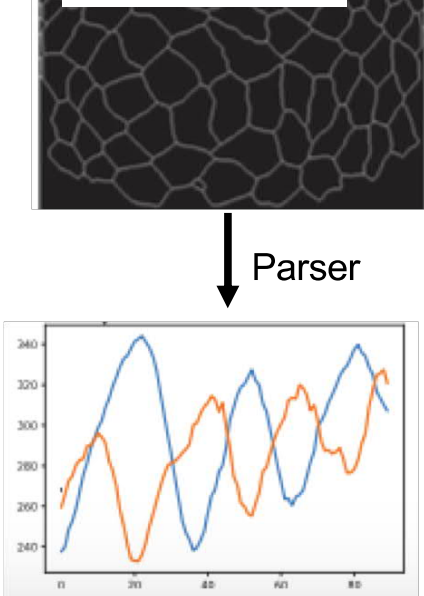

Segmented image
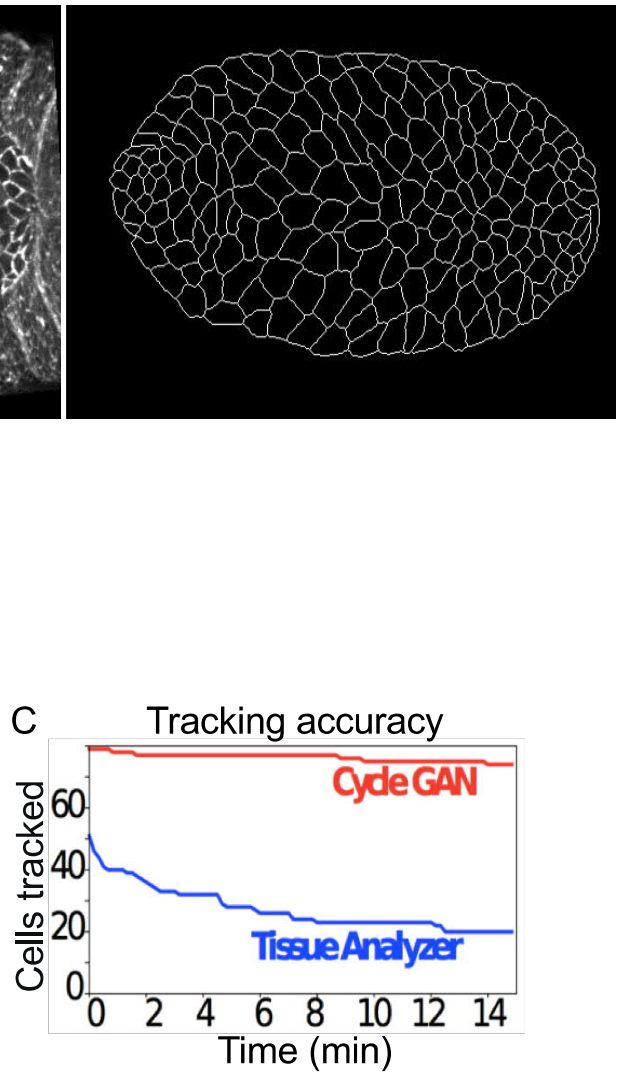

D

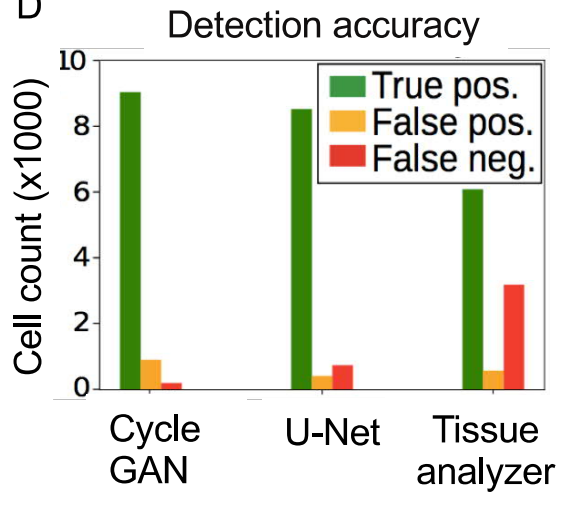

Fig 3.6 Deep learning neural network-based Segmentation approach. (A) Example of segmented AS cells in stage 14 wild-type embryos (image shown on the right side) from the raw maximal intensity projection image (shown on the left). (B) Pipeline for computational image analysis and image segmentation by cycle-consistent generative adversarial networks (GAN), trained with manually created ground truth images. (C) Comparison of tracking accuracy of Cycle-GAN approach and Tissue Analyzer, in the same AS movie. (D) Performance 
comparison between Cycle-GAN segmentation and two state of the art methods (U-Net and watershed-based segmentation from the Tissue Analyzer) during AS oscillations.

To circumvent this requirement of large training dataset, we used generative adversarial networks (GAN) as a deep learning approach for image segmentation. This segmentation approach works as reliable as the currently best machine learning method (U-Net), but requires only single ground-truth data set and one initial training session (Fig 3.6, D). Reliable segmentation was achieved also for the images with low signal-to-noise ratio using Cycle-GAN segmentation approach. Tracking accuracy via Cycle-GAN approach is robust and accurate also during dynamic cell behavior (Fig 3.6, C). However, as a limitation to this approach, the waviness of the cell borders could not be reconstructed via segmentation. Therefore, this approach was used to quantify and analyze total cell area and cell orientation dependent parameters.

Dynamic reconstruction of AS allowed us to follow the cell dynamics (coordinated oscillatory cell behavior) and morphological changes (spatiotemporal changes in anisotropy of the cells) over time for each and every cell in the AS. 


\subsection{TMC is involved in the maintenance of cell morphology and isotropy in the AS epithelium}

During this study, I mainly focused on understanding the functional mechanism of the putative MS channel TMC in detail. Loss of TMC did not have a large impact on the development of the embryo, yet the cuticle phenotypes in unhatched embryos indicated its involvement in the maintenance of epithelial integrity. Unhatched embryos (although in low percentage-18\%) showed morphological defects during stage 11-14. Deformed AS and the epidermis tissue morphology suggested that $T M C^{\Delta}$ mutant embryos, plausibly stop developing at this stage during embryogenesis (Fig 3.7).

To analyze detailed morphological cell dynamics in TMC depleted embryos, E-CadherinGFP was expressed from the endogenous locus in $T M C^{\Delta}$ embryo. E-Cadherin GFP marks the epithelial cell junctions, and allow visualization of dynamic epithelial rearrangement during morphogenesis. Time-lapse recordings of the live wild-type and $T M C^{\Delta}$ embryos (stage 13-14) were done. AS cell dynamics (pulsing/oscillatory behavior of AS cells) were followed over time, starting from the end of germ-band retraction to the initiation of the DC.

A
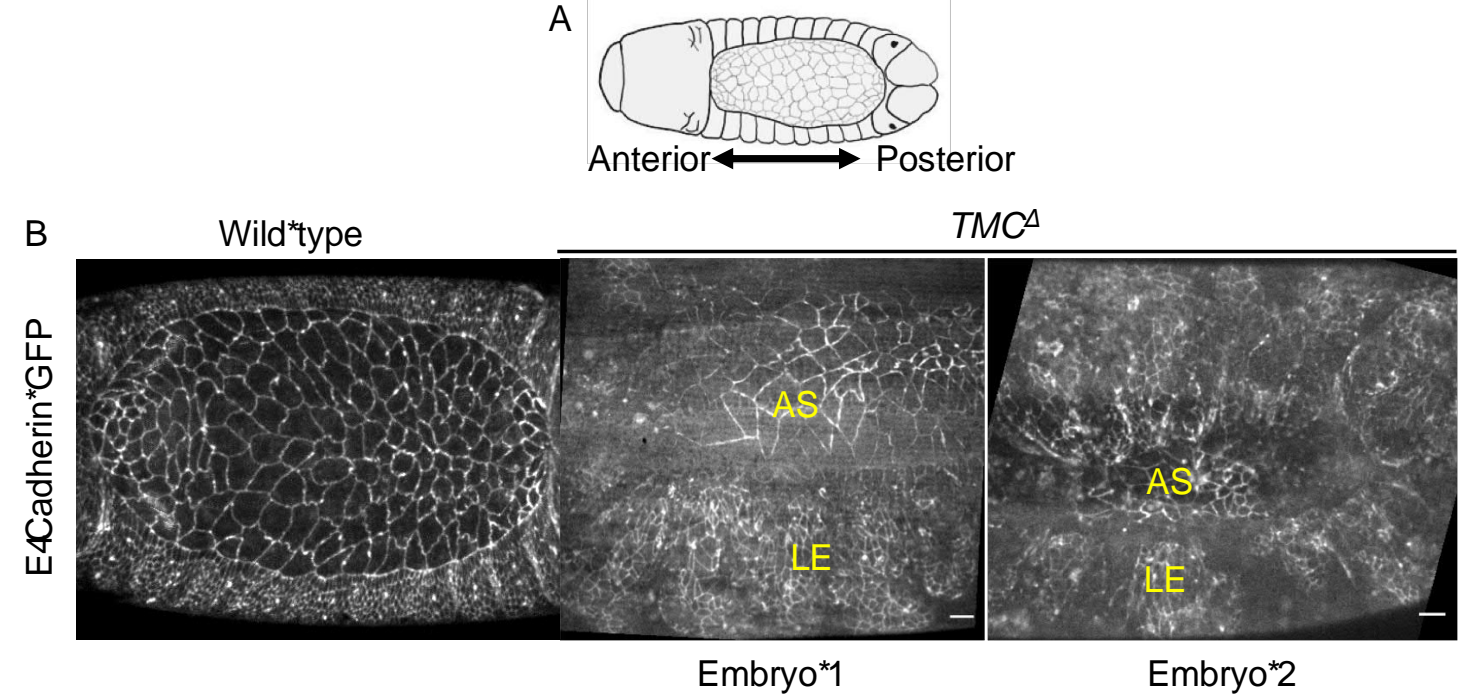

Fig 3.7 Loss of epithelial integrity in unhatched $T M C^{\Delta}$ embryos. (A) Embryo orientation as shown in the scheme. (B) $T M C^{\Delta}$ embryos (embryo 1 and embryo 2) had deformed amnioserosa (AS) tissue and lateral epidermis (LE). Scale bar=10 $\mu \mathrm{m}$. (All Images are maximal intensity projections of multiple z-stacks).

In one of the earlier studies from our group, it was shown that during the germ-band extension morphogenetic rearrangement of cells via cell intercalation in the epidermis is impaired in xit mutant embryos. xit encodes for a glucosyltransferase enzyme and is 
involved in glycosylation of E-Cadherin. It was also reported that loss of xit affects ECadherin dynamics (Zhang et al., 2014). For this study, xit mutant embryos were used as a reference to which I compared the phenotypes of embryos TMC depleted embryos in the AS.

\subsubsection{AS cells display anisotropic morphology in $T M C^{\Delta}$ and $x i t$ mutant embryos}

In wild-type embryos, AS cell morphology was highly anisotropic during germ-band retraction phase. These elongated AS cells gradually became isotropic, as the germ band retraction was complete and the AS moved dorsally at the end of stage 13 (Fig 3.8, C). This morphological transition of the AS has been reported before however, how the AS cells are able to induce this transition is not understood well (Lynch et al., 2013). Unlike wild-type, in $T M C^{\Delta}$ and xit embryos the AS cell remained comparatively anisotropic even after the dorsal ward movement of AS was complete (Fig 3.8, B; Fig 3.9, C, D).
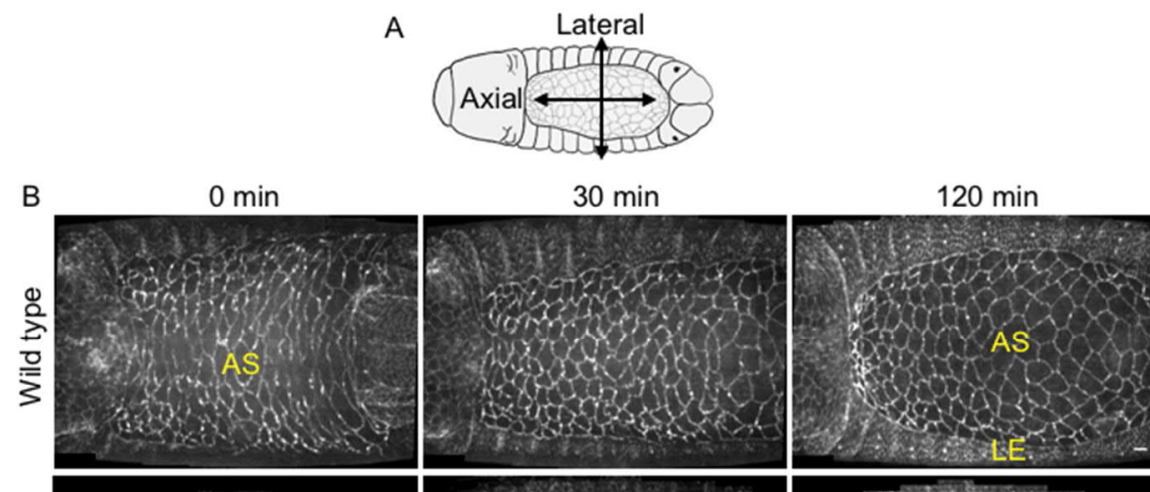

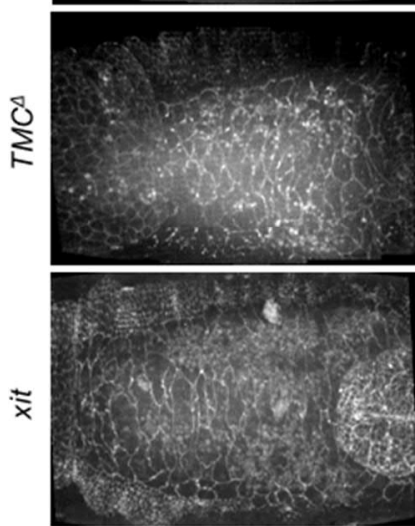

Germ-band retraction

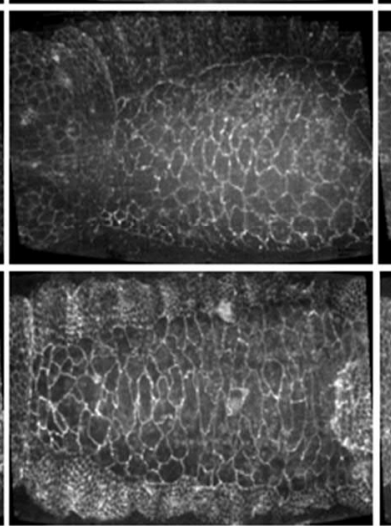

Early dorsal closure

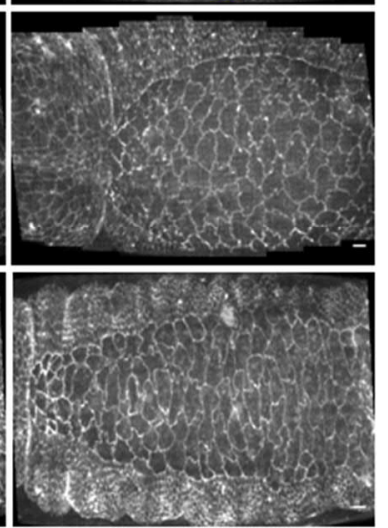

Onset of closure

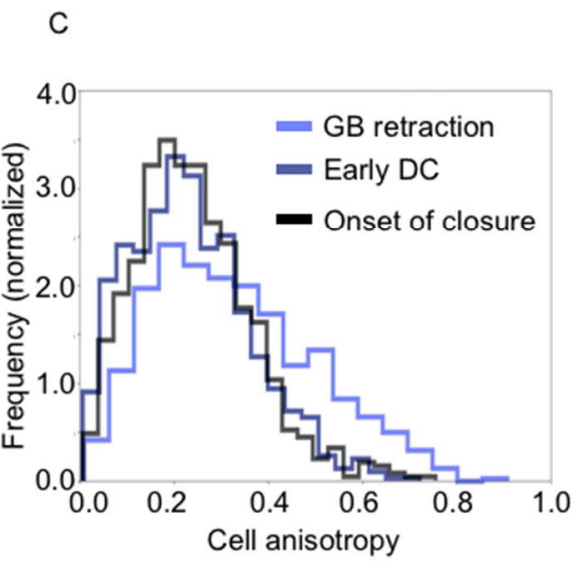

Fig 3.8 AS cells in $T M C^{\Delta}$ and xit embryos remain anisotropic post-germ-band retraction. (A) Scheme shows orientation of the embryos in anterior to the posterior manner (axial and lateral are orientation of the cells with respect to the anterior-posterior axis). (B) Specific time frames from the time-lapse recording of live embryos show the transition of AS cells from anisotropic (end of germ band retraction stage) to isotropic morphology (early DC stage) in wild-type ( $1^{\text {st }}$ panel). In $T M C^{\Delta}$ and xit embryos, most of the AS cells remained anisotropic after the germ-band retraction was complete (quantified in Fig 3.9). Amnioserosa (AS) and lateral 
epidermis (LE) is labelled in yellow. Stages corresponding to the time points: 0 min=End of Germ-band

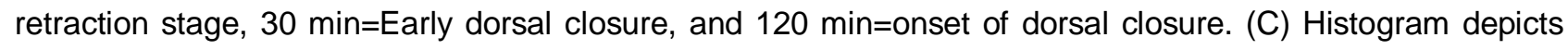
normalized frequency distribution of the degree of average anisotropy in the AS cells in wild-type embryos from germband extension to initiation of dorsal closure $(\mathrm{N}=436$ cells). Scale bar=10 $\mu \mathrm{m}$. (All images are maximal intensity projections of multiple z-stacks).

Images acquired at higher magnification (using 40X objective) clearly showed that anisotropic shape of the AS cells were stretched towards the lateral epidermis in $T M C^{\Delta}$ and xit embryos (Fig 3.10, A). This stretched cell morphology was prominent in the xit embryos.

I performed time-lapse recordings in multiple embryos, starting from the end of the germband retraction stage until the initiation of the DC (identified by the meeting of the lateral epidermis at the anterior front of the AS). Multiple time frames (including z-stacks) were acquired, starting from time aligned germ-band retraction stage until the initiation of the DC. Images were acquired at the interval of $35 \mathrm{~s}$ per frame (for $1.5 \mathrm{~h}$ in wild-type and $T M C^{\perp}$, and for 3.5h in xit embryos). Based on the distance between the germ-band and the cephalic furrow of the embryo, during germ-band retraction, I chose a time point. All embryos from wild-type, $T M C^{\Delta}$ and xit were aligned to this time-point (referred to as the end of germ-band retraction stage, shown at 0 min in Fig 3.8, B).

A

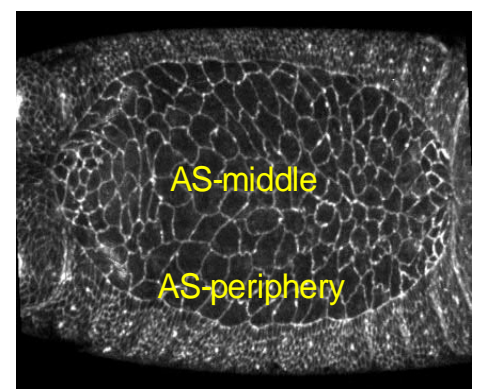

D

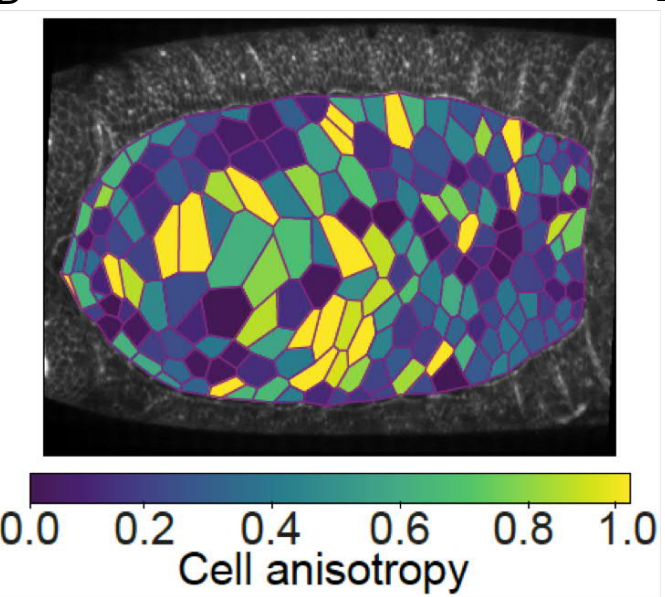

B

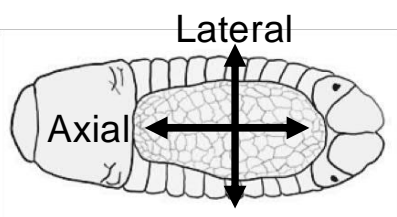

C

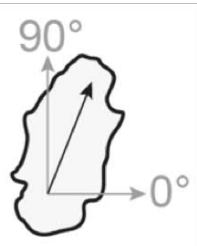

Anisotropy of AS cells during early DC stage

E

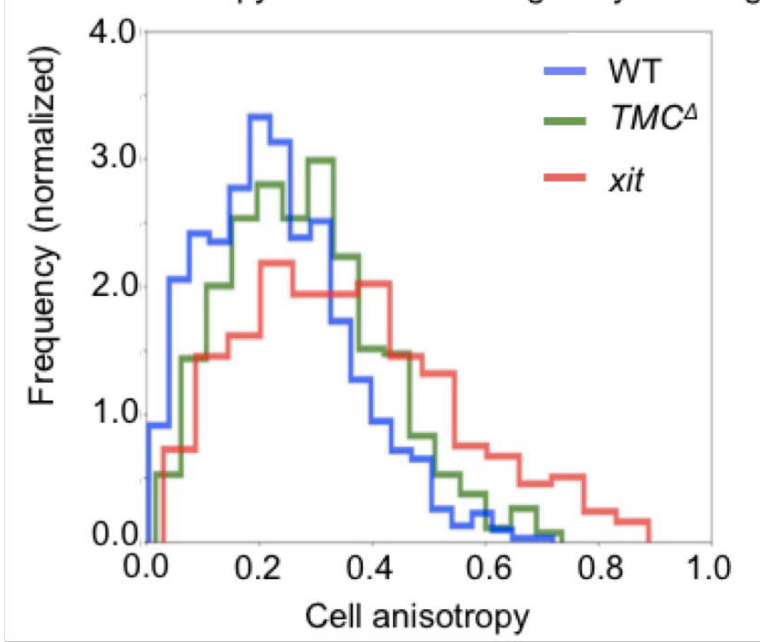


Fig 3.9 Quantification of spatiotemporal change in anisotropy of the AS cells from germ-band retraction to early DC stage. (A) Quantification was done selectively and separately for the cells located in the middle (area marked in blue) and at the periphery (area marked in red) of the AS, by choosing a selective ROI. (B) Scheme shows the orientation of the cell axis with respect to the anterior-posterior axis of the embryo. (C) AS cell elongation measured. (D) Heat map depicting isotropic and anisotropic cell shapes. On the scale of anisotropy 0.0 represents a circle and 1.0 represents a straight line. (E) Histogram depicts normalized frequency distribution of the AS cells displaying the degree of average anisotropy in the cells located in the middle of the AS in wild-type, $T M C^{\Delta}$ and xit embryos during early DC stage. $\mathrm{N}=436,317$ and 238 cells in 8 wild-type, $7 T M C^{\Delta}$, and $7 x i t$ embryos respectively.

Time-lapse images were segmented in order to proceed with large-scale computational analysis (as discussed in section 3.4, Fig 3.6, B). We quantified the anisotropy or cellelongation using a nematic tensor characterizing the magnitude of the elongation (Etournay et al., 2018). Anisotropy in the AS cells were computed independent of lateral and axial axis orientation (Fig 3.9, C, D).

Quantification of anisotropy in AS cells over multiple embryos showed that wild-type AS cells display anisotropic morphology during germ-band retraction, and eventually became isotropic during early DC stage when the complete amnioserosa tissue is exposed dorsally (Fig 3.8, B, C). However, unlike wild-type, the cells in $T M C^{\Delta}$ and xit, remained comparatively anisotropic even after completion of germ-band retraction and during early DC stage (Fig 3.9, C, D).

Interestingly, I also found that, cells located in the middle of the AS tissue had increased anisotropy compared to the cells at the periphery i.e. at the boundary of AS and epidermis in $T M C^{\Delta}$ and xit embryos. AS cells in the middle were unable to induce isotropic morphology, post-germ-band retraction in $T M C^{\Delta}$ and xit embryos. However, AS cells at the periphery stayed comparatively isotropic throughout their transition from germ-band retraction to DC stage (data shown in appendix section). This phenotype was evident during the end of germband retraction stage but more obvious during the early DC stage. In order to separately quantify the cells in the middle and at the periphery of the AS, selective region of interest (ROI) were chosen. The quantification was done over 8 wild-type, $7 T_{M C}{ }^{\Delta}$, and 7 xit embryos (Fig 3.9, C, D). 


\subsubsection{AS cell junctions display wavy morphology in $T M C^{\Delta}$ and $x i t$ mutant embryos}

Unlike the straight junctions in the wild-type embryos, a distinctly wavy and undulating morphology of cell junctions was observed in $T M C^{\Delta}$ and xit embryos (Fig 3.10, A). In order to quantify the waviness of the junctions in wild-type, $T M C^{\Delta}$ and xit embryos, a ratio between the total length of the junction (referred to as bond) and distance between two tricellular junctions (referred to as nodes) were taken (Fig 3.10, B).
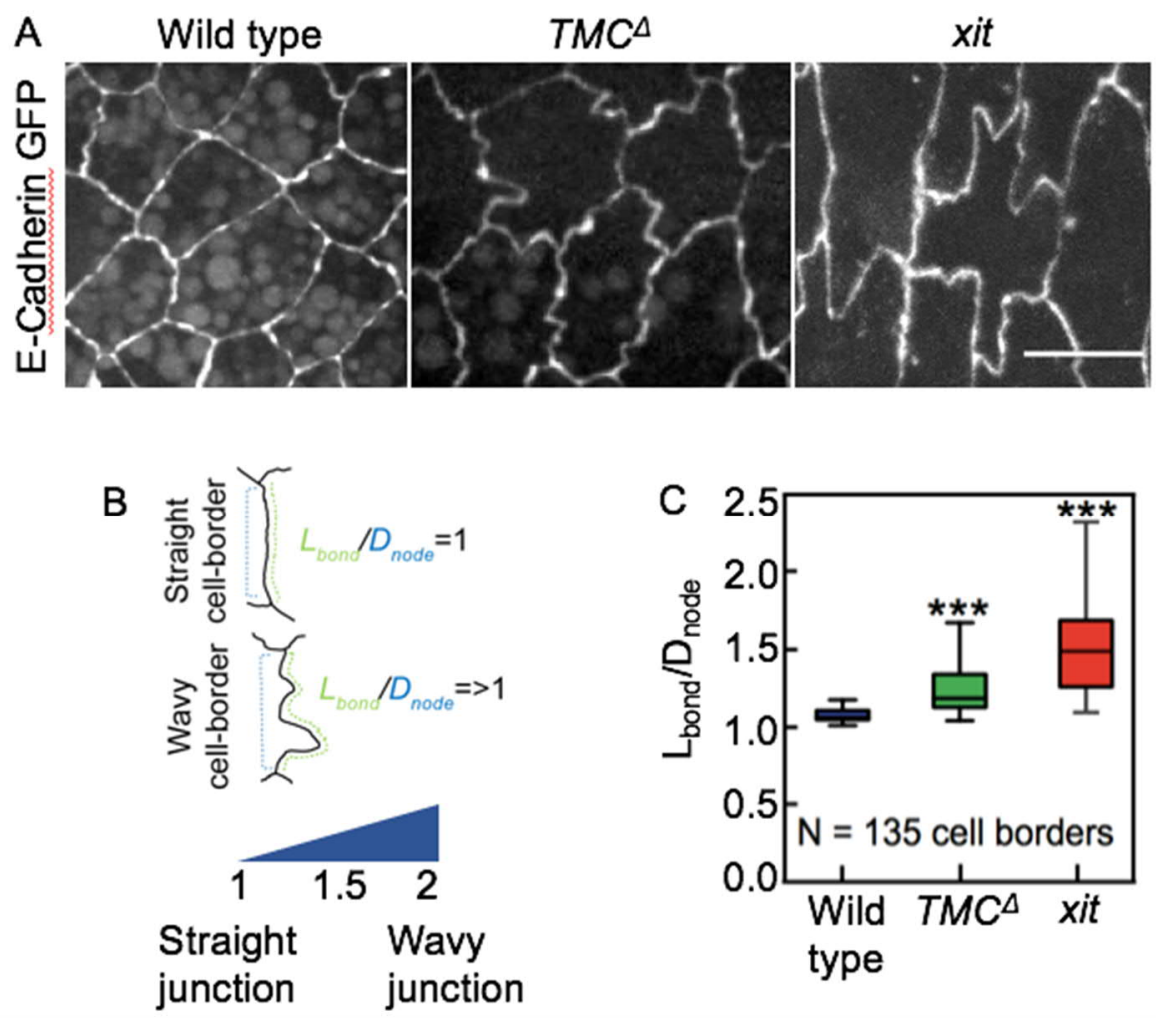

Fig 3.10 Quantification for cell junction waviness. (A) Images show AS cells in wild-type (straight junctions), $T M C^{\Delta}$ and $x$ it (wavy junctions) (B) Waviness was quantified as a ratio between the distance between the node and the length of bonds. On the scale of $1-2,1$ refers to straight junctions and $\geq 1.5$ refers to wavy junctions (as shown in the scheme). (C) Boxplots show mean \pm SEM $(\mathrm{p}<0.005) . \mathrm{N}=135$ cell borders in 3 embryos for Wild-type (shown in blue), $T M C^{\Delta}$ (shown in green) and xit (shown in red). Scale bar=10 $\mu \mathrm{m}$.

Waviness of the junctions were quantified in 135 cell-borders, over 3 embryos and for each genotype. There was a significant increase in the degree of waviness in AS cell junctions in $T M C^{\Delta}$ and $x i t$ embryos, compared to the wild-type. xit displayed higher degree of waviness at the junction compared to wild-type as well as $T M C^{\Delta}$ (Fig 3.10, C). 


\subsubsection{Total number of AS cells are reduced in $T M C^{\Delta}$ and xit embryos}

The total number of cells in complete AS tissue was measured which suggested that there was a significant decreased in the total number of AS cells in the $T M C^{\Delta}$ and xit embryos compared to the wild-type. Total cell count in the AS tissue was taken from wild-type, $T M C^{\Delta}$, and xit embryos at a fixed time-point during the early DC stage, and plotted as a boxplot (Fig 3.11). Each dot in the boxplot represent one embryo. Quantification for cell count was done for 8 wild-type, $7 T M C^{\perp}$, and 7 xit embryos.

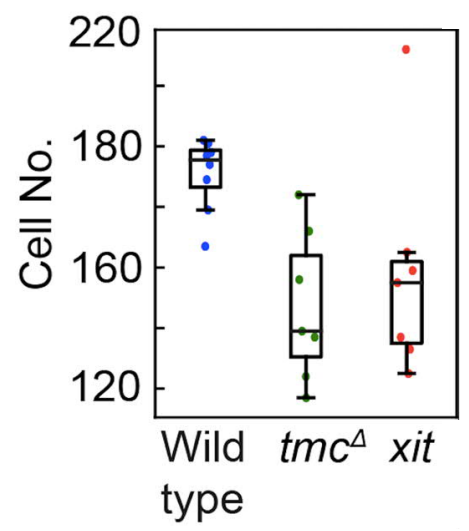

Fig 3.11 Total cell-count in the AS. Boxplot indicate the total number of AS cells in each embryo (mean \pm variance). Each dot represents one embryo. $\mathrm{N}=8$ wild-type (blue), $7 T M C^{\Delta}$ (green), and 7 xit (red) embryos.

\subsection{Coordinated oscillatory cell behavior and intercellular coupling between AS cell-pairs is affected in $T M C^{\Delta}$ and xit mutants}

AS cells exhibit continuous oscillations during DC. I investigated, if the loss of $T M C^{\Delta}$, and xit has any effect on the coordinated cell behavior of the AS cells. Continuous oscillations in the AS cells during the initial stationary stage of the DC are coordinated with their neighbors (discussed in detail in section 1.3.2). I chose temporal oscillatory cell dynamics, and intercellular coordination of cell-pairs linked by junctions, as a parameter to investigate coordinated cell behavior in wild-type, $T M C^{\Delta}$, and xit embryos. To study the dynamic oscillatory behavior of the AS cells, in an unbiased manner, large scale imaging, segmentation, and computational analysis were performed. I performed time-lapse recordings, in the stationary phase of oscillation of the AS cells during early DC stage embryos. As reported previously, the AS cells contract with a periodicity of $230 \pm 76$ s (Solon 
et al., 2009; Blanchard et al., 2010). Therefore, I acquired time-lapse at the rate of 1 frame per 35 s to include complete oscillatory phases of the AS cells.

\subsubsection{Neighbor dependent, non-autonomous oscillations are faster in the AS cells in $T_{M} C^{\Delta}$ and $x i t$ compared to the wild-type}

Oscillatory cell dynamics were analyzed specifically during the stationary phase of the AS oscillations, when the total area of the AS tissue is maintained and remain constant. We estimated the period of oscillation in AS cells directly from a Fourier transformation on the cell area trajectory over time. Periods from the autocorrelation function were indicated as a histogram plot. Dashed lines in the plot depict the median (Fig 3.12, C). The major period of oscillation was determined from the autocorrelation function of the cell area trajectories. The first peak represented the major period in the autocorrelation function in the wild-type, $T M C^{\Delta}$, and $x i t$ (Fig 3.12, B).

We found that AS cells in the wild-type embryos oscillate with a period of $5.15 \mathrm{~min}$ (consistent with the previous reports (Solon et al., 2009)). However, oscillations were faster in $T M C^{\Delta}$ (4.05 $\mathrm{min}$ ), and xit (2.55 $\mathrm{min}$ ) compared to the wild-type (Fig 3.12, B), suggesting the involvement of $T M C^{\Delta}$, and xit in the regulation of dynamic cell behavior in the AS.

A

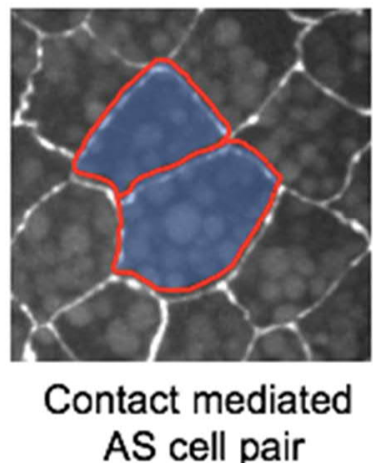

AS cell pair
B

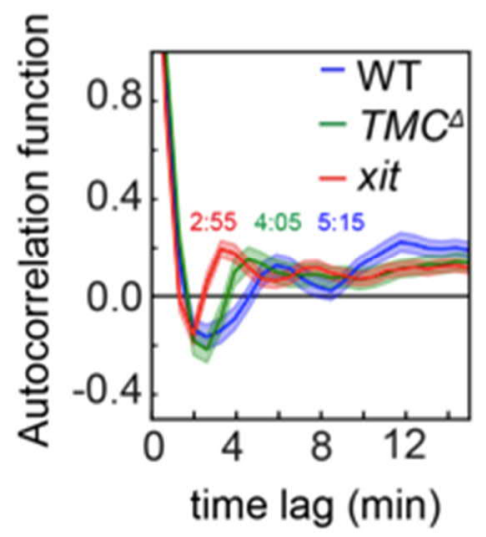

C
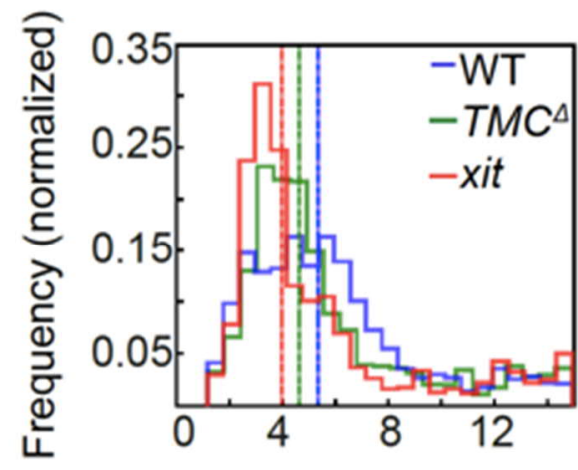

Oscillation period ( $\mathrm{min})$

Fig 3.12 Oscillatory dynamics of AS cells in wild-type, $T M C^{\perp}$, and xit. (A) Contact-mediated AS cell-pair. (B) Curves represent the dynamics of the cell oscillations. The first peak represents the major period in the autocorrelation function. Period for $T M C^{\Delta}$ and xit was significantly faster compared to the wild-type. (B) Periods from the autocorrelation function of the cell area trajectory. Dashed line represents median for wild-type (5.20 $\mathrm{min}$ ), $T M C^{\Delta}$ (4.38 min), xit (3.59 min). Statistical significance by Kolmogorov-Smirnov Test, $p<0.00001 . \mathrm{N}=8$ wild-type, $7 T M C^{\perp}$, and 7 xit embryos. 


\subsubsection{Distribution of coupling types and intercellular coordination is affected in $T M C^{\Delta}$ and xit mutants}

In the AS, contact-mediated cell-pairs were temporally coupled during the oscillations (Fig $3.13, A)$. In order to characterize the coupling type of the AS cell-pairs, intercellular coordination of AS cell contraction was analyzed using iso-correlation subsampling. From the entire population of the reconstructed cell-pairs, maximal subsamples exhibiting positive or negative correlations were selected. The cell-pairs physically linked to the neighboring cell via the cell junctions, were found to be positively (correlated), negatively (anti-correlated) or neutrally coupled (Fig 3.13, B-E). For these uniform ensembles of cell-pairs, ensemble averaged cross-correlation functions were calculated. The correlation coefficient was analyzed in order to depict the strength of the relationship between positively, negatively and neutrally coupled cell types (Fig 3.13, B).

In wild-type embryos, there was approximately equal distribution of positively and negatively coupled cell-pairs in the AS (positive/negative cell-pairs ratio=0.99). Interestingly, this balance was shifted in $T M C^{\Delta}$ with a significant decrease in the population of positively coupled cells types (positive/negative cell-pair ratio=0.31) (Fig 3.13 C-E). However, the strength of intercellular coordination of cell-pairs was not affected in $T M C^{\Delta}$ embryos as the ratio between significant/insignificant correlations in $T M C^{\Delta}(0.29)$ was comparable to wildtype (0.27) (Fig 3.13, E).

In xit mutants, all aspects of correlation were affected including cell-pair coupling as well as the overall strength of the correlation. There was a decrease in both positively and negatively coupled cell-pairs in the AS (Fig $3.13 \mathrm{C}-\mathrm{E}$ ). The ratio between significant/insignificant correlations in xit was $=0.22$, significantly lowered, compared to the wild-type. 
A
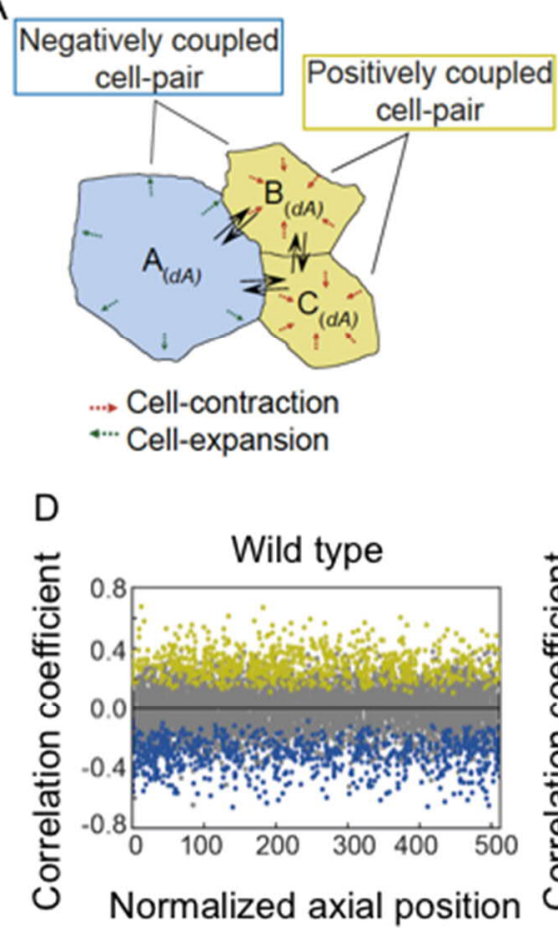

B
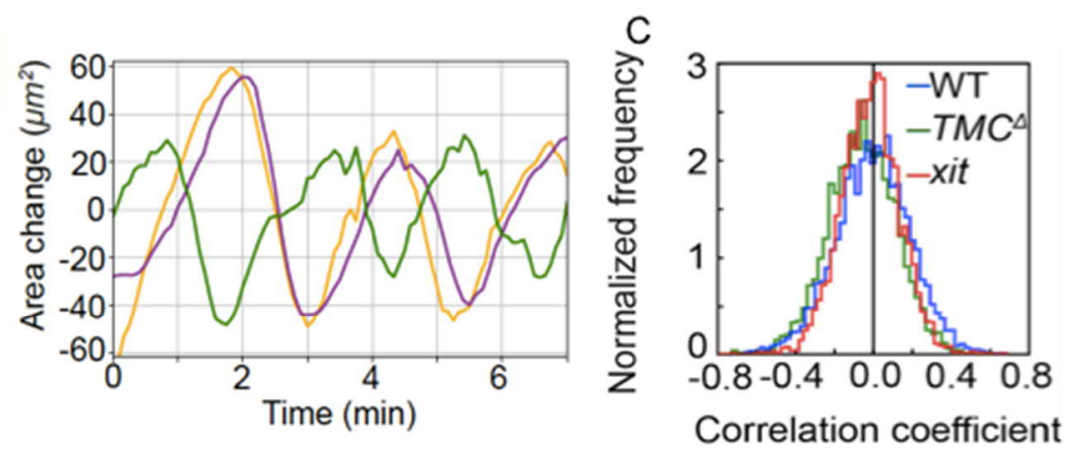

$\mathrm{E}$
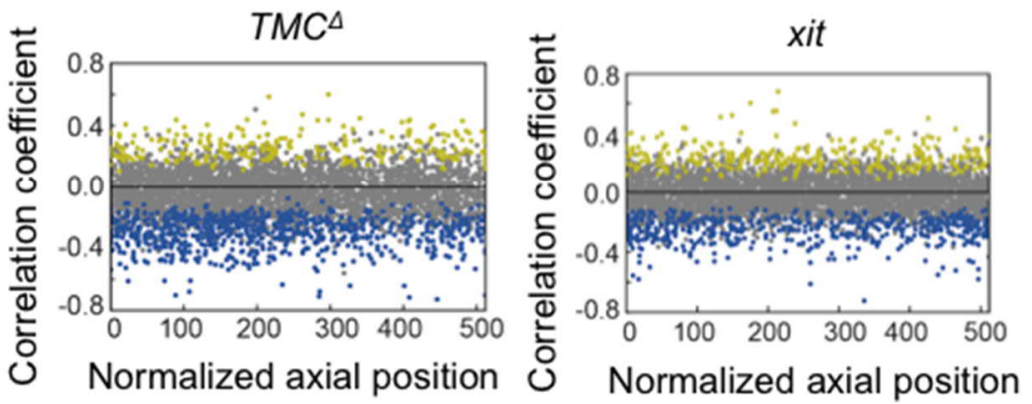

- Negative Positive Non-significant

\begin{tabular}{|c|c|c|c|c|c|}
\hline Type & Positive & Negative & Neutral & $\begin{array}{c}\text { positive/negative } \\
\text { correlations }\end{array}$ & $\begin{array}{c}\text { sig/insig } \\
\text { correlations }\end{array}$ \\
\hline WT & 795 & 805 & 5774 & 0.99 & 0.27 \\
\hline$T M C^{\Delta}$ & 254 & 809 & 3570 & 0.31 & 0.29 \\
\hline xit & 324 & 541 & 3849 & 0.59 & 0.31 \\
\hline
\end{tabular}

Fig 3.13 Intercellular coupling between AS cell-pairs in wild-type, $T M C^{\perp}$, and xit. (A) AS cell-pair are coupled in positive and negative manner. (B) Curves show change in AS cell area plotted over time for cells oscillating in positively correlated and negatively correlated manner. (C) The plot shows correlation coefficient for all negatively and positively correlated cells pairs in the wild-type, $T M C^{\Delta}$, and xit. (D) Positively and negatively coupled cell-pairs are shown with respect to the normalized axial position in the wild-type, $T M C^{\Delta}$, and xit embryo. Cell-pairs were selected according to their correlation to a reconstructed neighboring cell. $(E)$ Table shows total ratio of positive/negative correlations, and the ratio of total significant/insignificant correlations in the AS of wild-type, $T M C^{\Delta}$, and xit embryo. Tracked cell-pairs: wild-type (7374), $T M C^{\Delta}$ (4633), and $x$ it (4714). $\mathrm{N}=8$ wild-type, $7 \mathrm{TMC}^{\perp}$, and 7 xit embryos. 


\subsubsection{Spatial pattern of coupling types of AS cell-pair}

Interestingly, we found that coordinated cell-pairs were not only coupled as a specific type but were also distributed in a position-based manner in the wild-type embryos. We attempted to generate a spatioal map of cell-coupling types in the AS tissue. In order to generate spatial distribution of positively and negatively coupled cell-pairs, the correlation coefficients were taken in to account (significant and insignificant).

Wild type

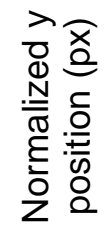

Normalized $x$ position $(p x)$

$T M C^{\Delta}$

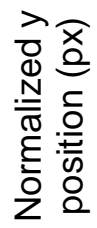

Normalized $\mathrm{x}$ position $(\mathrm{px})$

xit

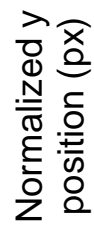

Normalized $x$ position $(p x)$

Fig 3.14 Spatial 2D map of cell coupling types in AS in the wild-type, $T M C^{4}$, and xit embryo. All the datapoints for the correlation coefficient were averaged over many embryos in a position-based manner. Tracked cell-pairs: wild-type (7374), $T M C^{\Delta}$ (4633), and xit (4714). $\mathrm{N}=8$ wild-type, $7 T M C^{\Delta}$, and 7 xit embryos.

AS cell-pairs in wild-type were able to maintain a balance between positively coupled (red) and negatively coupled cells (blue), as they were evenly distributed throughout the AS. However, there was a significant reduction in positively coupled cell types in $T M C^{\Delta}$, which resulted in shift of the balance towards the negatively coupled cell-types (indicated by 
increased blue area in the map). There was an overall reduction in both coupling types in xit embryos (Fig 3.14).

\subsection{AS cell junctions exhibit anisotropic force distribution in $T M C^{\Delta}$ and xit mutants}

Next, based on the observation of anisotropic shapes of AS cells in the $T M C^{\Delta}$ and xit mutants, I asked whether the mechanical properties of the cells are also affected. Increased waviness of the AS cell junction in $T M C^{\perp}$, and xit embryos also suggested a plausible change in the mechanical tension distributed within the AS cells (section 3.5.2). A commonly used approach to estimate mechanical tension experienced by the membrane/junction of the cell, is via laser ablation (Hutson et al., 2003). In order to reveal junctional tension, I did laserinduced junctional cuttings using a pulsed UV laser. Early DC stage Wild-type, $T M C^{\Delta}$, and xit embryos were chosen and individual AS junctions were selected. I made laser-induced cuts separately on the junctions with an axial or lateral orientation with respect to the anterior-posterior axis of the embryos (Fig 3.16; Fig 3.17). A single laser-induced cut was made in each embryo. $\mathrm{N}=10$ for each condition. The time-lapse recordings were done at the rate of $0.3 \mathrm{~s} /$ frame.

To calculate the recoil velocity, displacement of the adjacent $3 X$ vertices of the ablated junction were followed over time by using MTrack plugin in Fiji. Velocity was calculated as a function of time-post-laser cut, and the data were then fit using the equation for a viscoelastic element to the strain trajectory $\left(\right.$ strain $\left.=v / k^{*}\left[1-\exp \left(-k^{*} t\right)\right]\right)$. Here $v$ represented the recoil velocity and $k$ was the time-scale parameter. Initial recoil velocity was calculated from the recoil velocity of first $5 \mathrm{~s}$ after the laser-cut (Fig 3.15).
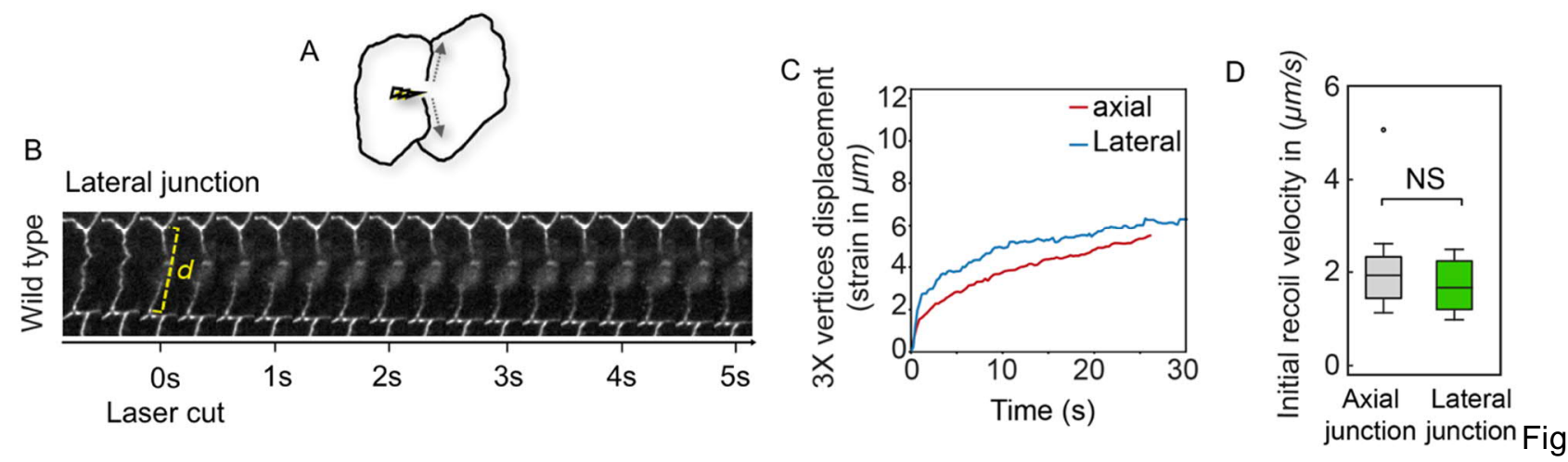

3.15 Quantification recoil velocity in axially vs. laterally oriented junctions in the AS cells. (A) Scheme for junction ablation experiment in laterally orientated junctions. (B) Kymograph indicate laterally oriented AS junction followed over time, post laser ablation, in wild-type embryos. (C) Displacement $d$ of the adjacent $3 X$ 
vertices of the selected junction was calculated and plotted as a strain trajectory. Curves indicate strain trajectory followed in 1 embryo each, for axial and lateral junction cut. Equation for viscoelastic element was fit to the strain theory to obtain recoil velocity. (D) Boxplots show quantification for the initial recoil velocities of axial and lateral junctions in Wild-type. Initial recoil velocity was calculated from the first $5 \mathrm{~s}$ time-frame of the displacement trajectory. $\mathrm{N}=10$ for each condition. Time resolution is $0.3 \mathrm{~s}$. Statistical significance by Student's T-test.

\subsubsection{Recoil velocity in the junctions with axial orientation}

First, I made laser induced cuts at the junctions oriented axially to the anterior-posterior axis of the embryo. Initial recoil velocity, in the axially oriented junctions in $T M C^{\Delta}$ and xit mutants, were comparable to the wild-type junctions (Fig 3.16, B-E).

A

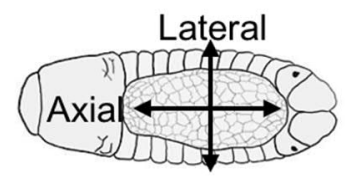

B

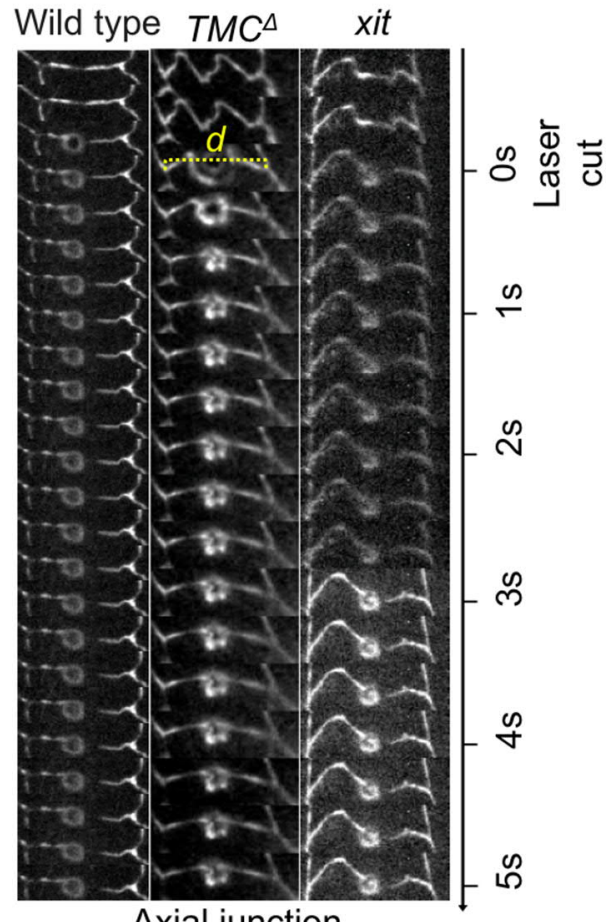

C

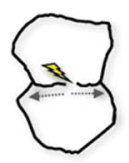

D

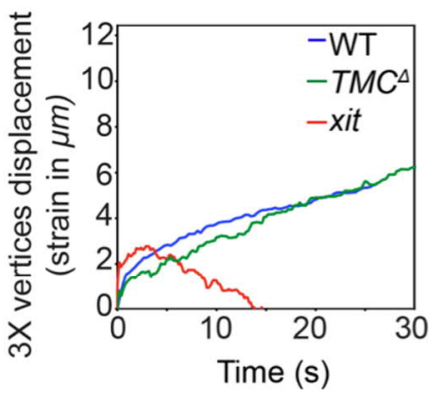

$\mathrm{E}$

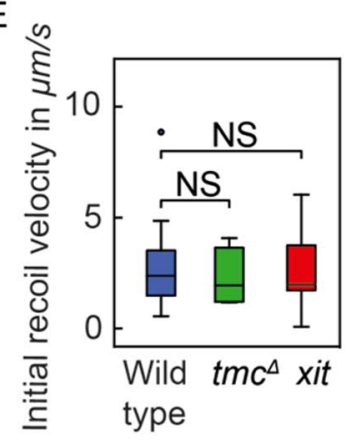

Fig 3.16 Recoil velocity at the axial junctions within the AS cells in the wild-type, $T M C^{\triangle}$, and $x i t$ embryos. (A) Scheme of AS. Arrows indicate the axial and lateral orientation of junctions with respect to the anterior-posterior axis of the AS. (B) Kymograph for ablation of a junction with axial orientation. (C) Scheme for junction ablation experiment in axially orientated junctions. Displacement $d$ of the adjacent $3 X$ vertices of the selected junction is followed over time (as indicated by the arrows). (D) Curves indicate $3 X$ vertices displacement trajectory followed in 1 embryo, for axial junction cut in wild-type, $T M C^{\Delta}$, and xit $(\mathrm{E})$ Boxplots show mean and variance for the initial recoil velocities of axial junctions in Wild-type (blue), $T M C^{\Delta}$ (green) and xit (red). $\mathrm{N}=10$ for each condition. Statistical significance by Student's T-test. 


\subsubsection{Increased recoil velocity in the laterally oriented junctions in $T M C^{\Delta}$ and xit mutants compared to the wild-type}

Unlike axial junctions, laterally oriented AS cell junctions showed significantly higher initial recoil velocity post laser-cut, in $T M C^{\Delta}$ and xit mutants, compared to the wild-type (Fig 3.17, $B-E)$.
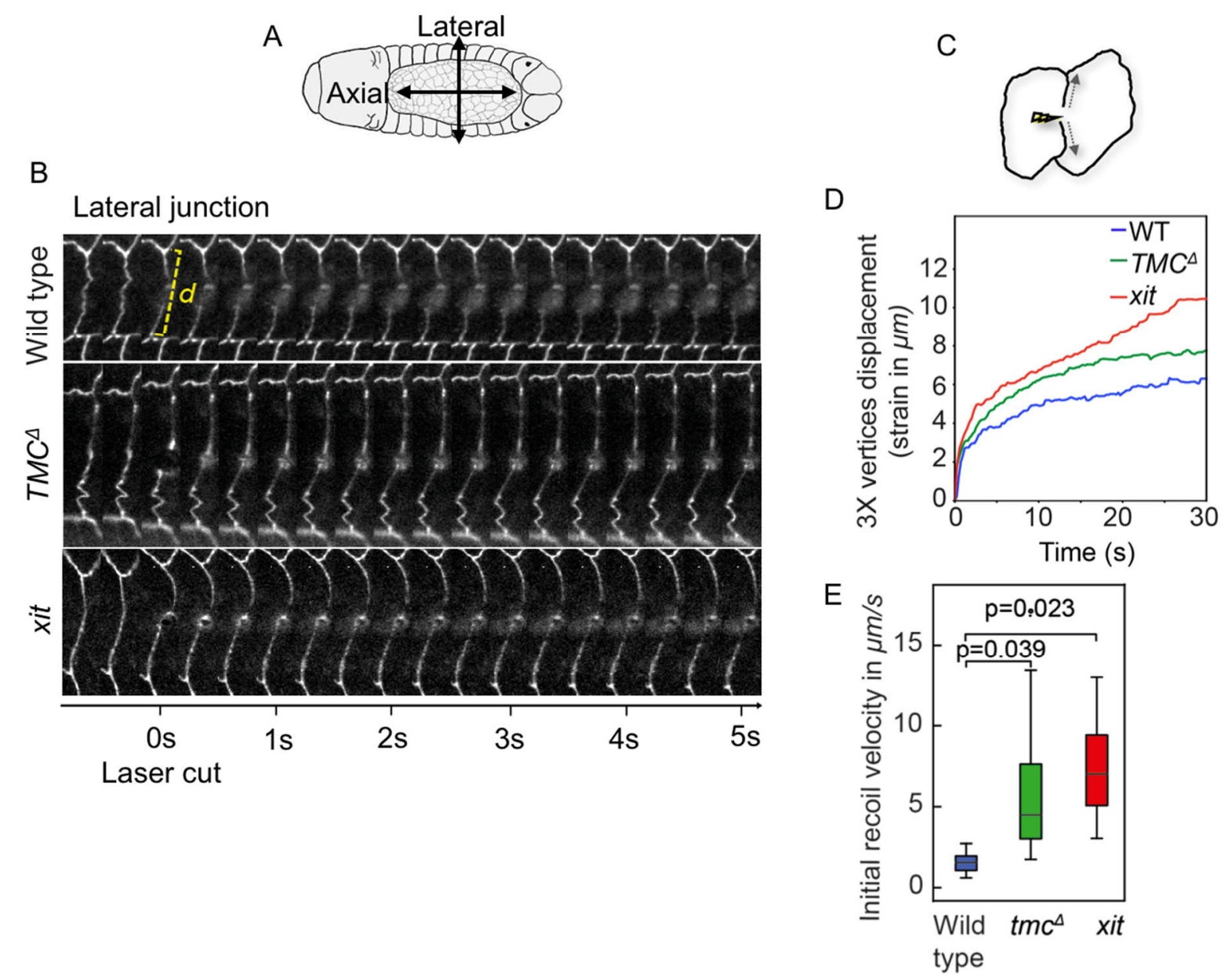

Fig 3.17 Laterally oriented junctions in $T M C^{\perp}$ and xit display higher recoil velocity compared to wildtype junctions. (A) Scheme of AS indicating the axial and lateral orientation of junctions with respect to the anterior-posterior axis of the AS. (B) Kymograph for ablation of a junction with lateral orientation. (C) Scheme for junction ablation using UV laser in laterally orientated junctions. Displacement $d$ of the adjacent $3 X$ vertices of the selected junction is followed over time (as indicated by the arrows). (D) Curves indicate $3 X$ vertices displacement trajectory followed in 1 embryo, for lateral junction cut in wild-type, $T M C^{\perp}$, and $x i t(E)$ Boxplots show mean and variance for the initial recoil velocities of lateral junctions in wild-type (blue), $T M C^{\Delta}$ (green) and $x$ it (red). $\mathrm{N}=10$ for each condition. Statistical significance by Student's T-test ( $\mathrm{p}$-value as indicated in the figure).

In wild-type embryos, recoil velocity did not change based on the orientation of the junction suggesting isotropic tension distributed within the AS cell junctions in wild-type (Fig 3.15). 
Also, there were no significant differences in initial recoil velocities, of the junctions with axial orientation in $T M C^{\Delta}$, and xit mutants compared to the wild-type (Fig 3.16). However, a two to three-fold increase in recoil velocity was detected in the junctions with lateral orientation, in $T M C^{\Delta}$ and xit mutants, compared to the wild-type (Fig 3.17). Higher tension in the AS cell junction with lateral orientation was consistent with the laterally stretched anisotropic morphology of the cells in $T M C^{\Delta}$ and xit mutants (Fig 3.8 and Fig 3.9). These evidences suggested a differential and anisotropic distribution of tension within the AS cell depending on the junctional orientation in $T M C^{\Delta}$ and xit mutants.

\subsubsection{Anisotropic tension at the actin cable in the wild-type}

Actin cable $(A C)$ is a contractile purse-string at the leading edge of the lateral epidermis. Differential distribution of tension at the $A C$ in a position-based manner is reported and wellstudied after the initiation of the DC in stage 14-15 embryos (Ducuing and Vincent, 2016; Kiehart et al., 2000a). However, position-dependent, tension distribution at the AC during early dorsal closure stage embryos (stage 13-14) remain vaguely understood. I attempted to address if this differential distribution of tension at the $A C$ already arises during early dorsal closure stage, in the wild-type embryos.

I performed laser-induced junctional cutting at the $A C$ of the early dorsal closure stage embryos. Selective cuts were made at the axial position (AC towards the anterior, cephalic furrow region) and lateral position of the $A C$ (aligned parallel to the lateral epidermis) (Fig $3.18, A) . N=10$ for each case in wild-type embryos.

Interestingly, I found that the $A C$ at the lateral position had increased recoil velocity compared to the axial position in the wild-type embryos. It has been reported before that lateral epidermis counteracts the forces produced by the AS and the leading edge (Kiehart et al., 2000a). This finding further suggested that despite the anisotropic forces exerted on the amnioserosa tissue via the AC, AS cells maintain isotropic morphology within the tissue in Wild-type embryos. Also, this suggests the presence of a mechanism in the AS cells for the maintenance of isotropic cell shape despite the anisotropic forces experienced by the tissue. Another evidence that indicated towards this property of AS, was the relatively stretched morphology of the cells at the leading edge of the epidermis (Fig 3.18, D). Epidermal cells at the leading edge became anisotropic as DC progressed. However, AS cells maintained their isotropic morphology. 


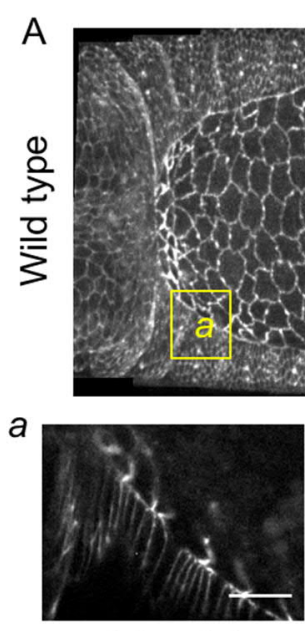

AC axial

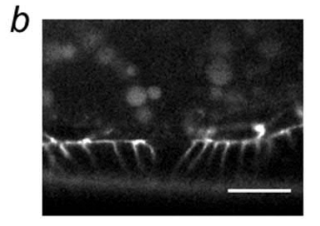

AC lateral
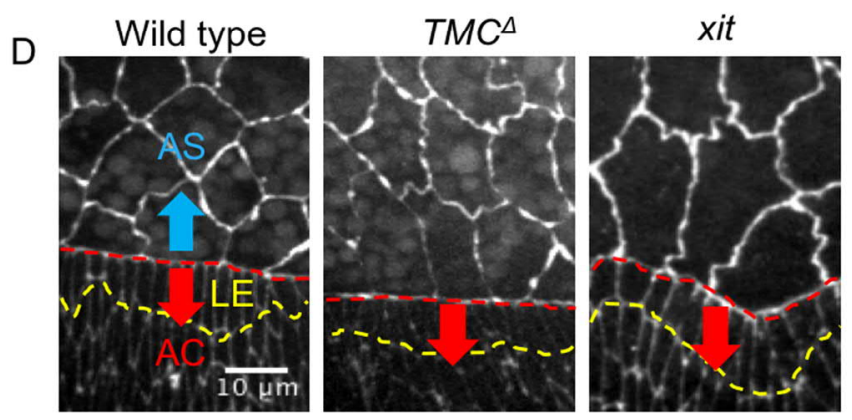

$\mathrm{B}$

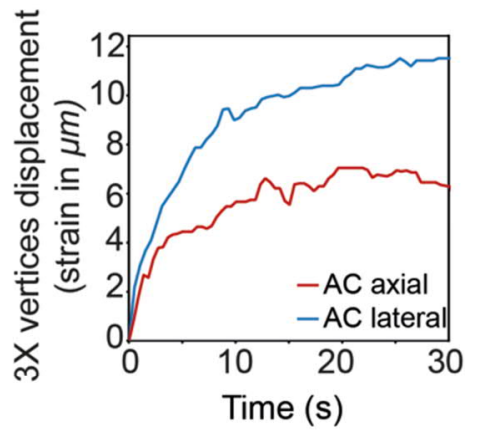

C

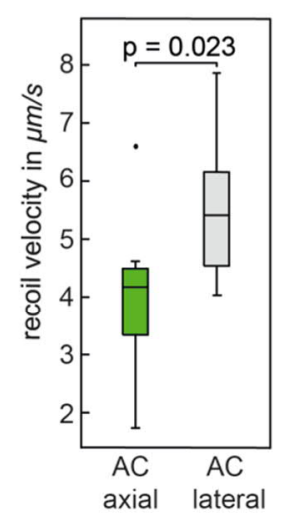

Fig 3.18 Anisotropic tension distribution at the AC. (A) Images show positions at the AC where laserinduced cuts were made. (a) Axial position at the AC, (b) lateral position at the AC. (B) Curves indicate $3 X$ vertices displacement trajectory followed in 1 embryo, for axial and lateral AC cut in wild-type. (C) Boxplot show mean and variance of the initial recoil velocity at axial (green) and lateral (grey) position of the AC in wild-type embryos. $\mathrm{N}=10$ for each condition. Statistical significance by Student's T-test ( $p$-value as indicated in the figure). (D) Images show the stretched morphology of the first row of epidermal cells at the leading edge (yellow), while AS cells maintain isotropy in the wild-type but not in $T M C^{\Delta}$ and xit. Scale bar=10 $\mu \mathrm{m}$. 


\subsection{Neighbor dependent $\mathrm{Ca}^{2+}$ mediated wound response is perturbed in $T M C^{\Delta}$ and $x i t$ mutant}

Next, I attempted to understand molecular aspects of the function of putative mechanosensitive ion channel TMC. Having known to affect $\mathrm{Ca}^{2+}$ conductance in sensory system, I asked if $\mathrm{Ca}^{2+}$ dependent responses are altered in $\mathrm{TMC}^{\Delta}$ embryos. Previously it has been reported that epithelial tissue damage induced coordinated neighbor cell responses. Therefore, I asked, if the neighbor-dependent wound response was affected in the $T M C^{\Delta}$, and xit embryos, in comparison to the wild-type. To investigate neighbor celldependent wound response, I performed cell ablation in individual cells in the AS (referred to as target cell), using pulsed UV laser (355nm). Early DC stage embryos expressing genetically encoded, myristoylated (membrane-bound) Calcium-sensor GCaMP6s (Chen et al., 2013) in the wild-type, $T M C^{\Delta}$, and xit background, were used for the wounding assay. Following the laser ablation of the cell, $\mathrm{Ca}^{2+}$ dynamics in the neighboring cells was followed and recorded for the next 5 min with the frame acquisition rate of $1 \mathrm{~s} /$ frame.

To follow the $\mathrm{Ca}^{2+}$ signaling dynamics, GCamp6s fluorescence was measured as mean intensity over time, at the membrane of the first neighboring cells that were in physical contact to the target cell (Fig 3.17, A). Fluorescence intensity was measured every 10s for 300 s in the wild-type, $T M C^{\Delta}$ and xit embryos (using Fiji). GCamp6s fluorescence intensity was normalized to the average intensity of the first 4 frames, prior to laser ablation $\left(\mathrm{I} / \mathrm{I}_{0}\right)$.

\subsection{1 $\mathrm{Ca}^{2+}$ dependent Myosin II accumulation following epithelial wounding}

I performed laser induced wounding in the target AS cell in embryos expressing GCaMP6s sensor. This triggered an instantaneous increase (within $\sim 10 \mathrm{~s}$ ) in the $\mathrm{Ca}^{2+}$ fluorescence signal at the membrane of adjacent cells in wild-type embryos (Fig 3.19). Wounding in wildtype embryos expressing Spg-Sqh GFP (GFP tagged to non-muscle myosin II regulatory light chain) displayed an increase in Myosin II intensity in the neighbors within $~ 1.5-2 \mathrm{~min}$ (Fig 3.19, B, C). Increased Myosin II levels at the wounded site, following sudden $\mathrm{Ca}^{2+}$ increase in the neighbors suggested $\mathrm{Ca}^{2+}$ induced accumulation of Myosin II at the wounded site following laser cut. 
A

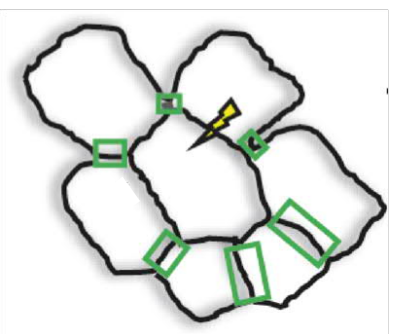

Target cell

Intensity measured at the neighboring junction
B

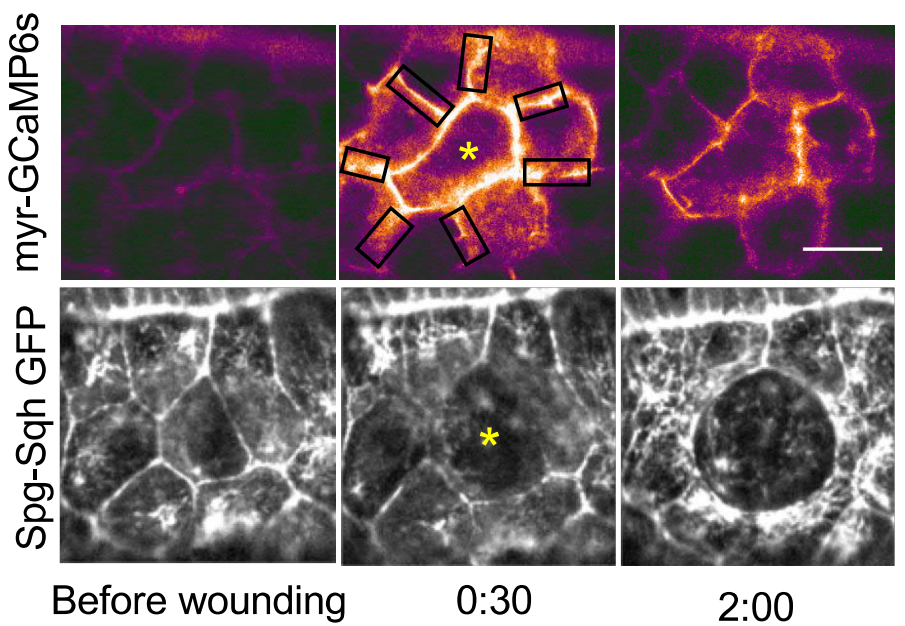

C

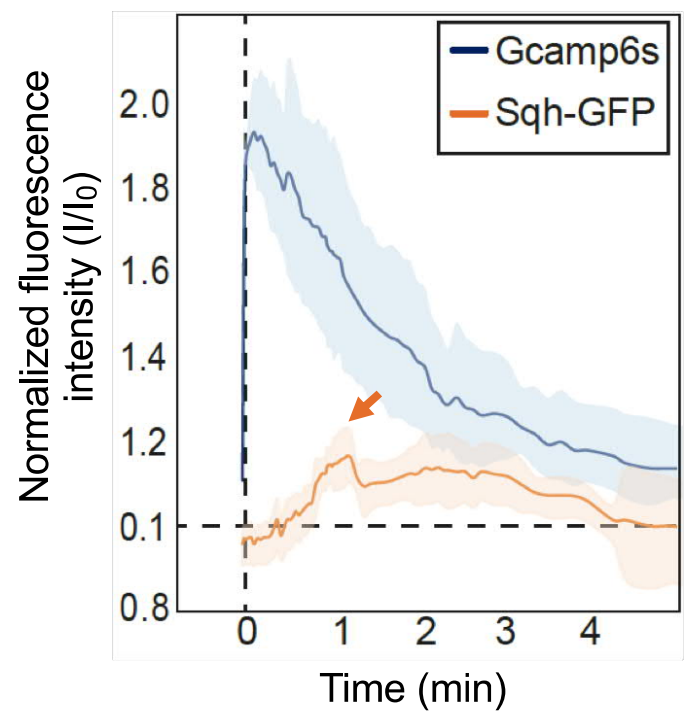

Fig 3.19 UV laser induced wounding triggers $\mathrm{ca}^{2+}$ dependent response in the neighbor cells in the AS tissue. (A) Experimental scheme for cell wounding (a single target cell is ablated by pulsed UV laser), followed by measurement of $\mathrm{Ca}^{2+}$ dependent response of the neighboring cells junctions. GCaMP6s sensor fluorescence is measured in the indicated areas of the junctions between the first neighbors (marked in green). (B) Time-points from the recording of before and after wound induction in individual AS cells in wild-type embryos expressing membrane-bound GcaMP6s sensor and Spg-Sqh GFP. Boxes indicate the area where fluorescence intensity is measured in the neighbors. (C) Normalized mean fluorescence intensity of the neighboring cell membrane plotted over time (solid curve) in embryos expressing membrane-bound GcaMP6s sensor (blue curve) and Spg-Sqh GFP (orange curve). Mean intensity was measured at all the junctions of the cells adjacent to the wounded cell (area indicated in the box). Mean fluorescence intensity was measured every 10 s for 300 s. Shaded area indicates the standard deviation of the mean. $\mathrm{N}=3$ for each condition. Scale bar $=10 \mu \mathrm{m}$. 


\subsection{2 $\mathrm{Ca}^{2+}$ dependent neighbor cell response to wounding is perturbed in $T M C^{\perp}$}

In $T M C^{\perp}$ embryos, the instantaneous increase of $\mathrm{Ca}^{2+}$ in the neighboring cells following wounding of the target cell, as a response to the wounding was significantly decreased compared to the wild-type (Fig 3.20, A). Also, this mild increase in $\mathrm{Ca}^{2+}$ levels stayed longer in the neighboring cells post wounding (data not shown). Perturbed neighbor-dependent $\mathrm{Ca}^{2+}$ response in the $T M C^{\Delta}$ embryos, indicated $\mathrm{TMC}$ mediated $\mathrm{Ca}^{2+}$ dependent response to the disruption of epithelial integrity.

\subsubsection{Neighbor dependent $\mathrm{Ca}^{2+}$ response to wounding is severely perturbed in xit}

To investigate $\mathrm{Ca}^{2+}$ dependent wound response by the neighboring cells in xit depleted embryos, laser-induced cuts were made in the xit germline clones expressing GCamp6s, under the control of AS tissue-specific Gal4 driver (AS-Gal4). As a result of this recombination, in the $50 \%$ of the embryos xit was depleted maternally as well as zygotically. However, in the remaining $50 \%$ of the embryos xit was only maternally depleted and had a zygotic rescue. Since these two pools of embryos were indistinguishable from each other during selection, I performed the laser cut experiment with multiple embryos in order to phenotypically differentiate between them $(\mathrm{N}=31)$.

Out of the total no. of embryos analyzed, in $58 \%$ of the embryos showed significant decrease in $\mathrm{Ca}^{2+}$ levels in any of the neighboring cells that were in contact with the wounded cell. These embryos showed an autonomous response to the tissue damage. In addition to this, in xit mutants neighboring AS cells continue oscillating upon wounding unlike in wild-type where neighboring AS cells slow down or stop their oscillations (data not shown). Remaining $42 \%$ showed a subtle and transient increase of $\mathrm{Ca}^{2+}$ in the neighboring cells and thus, a non-autonomous, neighbor dependent response, to the tissue damage. A subtle increase in normalized peak fluorescence intensity was observed in the embryos with non-autonomous response, at around 10-20s post-laser cut was observed as a result of this transient increase (Fig 3.21, B). Despite the weak transient increase in $\mathrm{Ca}^{2+}$ levels in the neighboring cells, in approximately half of the xit germline clones, total $\mathrm{Ca}^{2+}$ dependent response in xit embryos was significantly reduced in both genotypes. (Fig 3.20; Fig 3.21). 


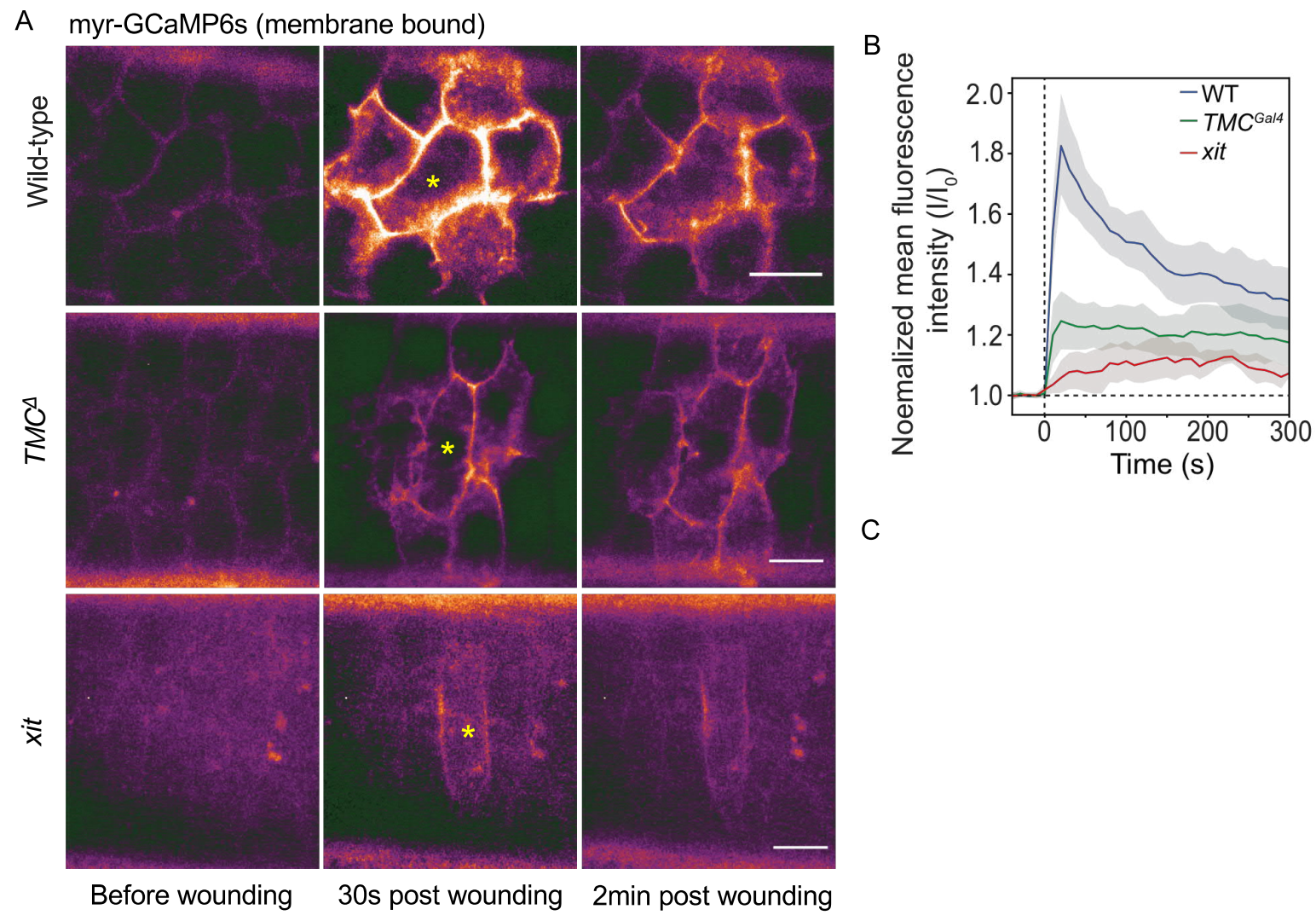

Fig 3.20 Wound induced neighbor dependent $\mathrm{Ca}^{2+}$ dynamics is perturbed in $T M C^{\Delta}$, and xit mutant. (A) Time-points from the recording of before and after wound induction in individual AS cells in wild-type, $T M C^{\Delta}$, and $x i t$. (The target cell is marked with a star) (B) Normalized mean fluorescence intensity of the neighboring cell junctions, was plotted over time (solid curve) for wild-type (blue), $T M C^{\Delta}$ (green), and xit (red). Shaded area indicates the standard deviation of the mean. Mean fluorescence intensity was measured every $10 \mathrm{~s}$ for $300 \mathrm{~s}$. $\mathrm{N}=10$ for each condition. (C) Boxplots show mean \pm SEM. Each dot represents one embryo. Normalized mean fluorescence intensity is compared in wild-type (1.60), $T M C^{\Delta}(1.14)$, and xit (1.02) at 10s post-laser cut. pvalue $<=0.005$ (Statistical significance by Student's T-test). $\mathrm{N}=10$ for each condition. Scale bar $=10 \mu \mathrm{m}$.

Overall, $\mathrm{Ca}^{2+}$ dependent wound response in the adjacent neighboring cells was severely perturbed in the absence of xit. Although the initial $\mathrm{Ca}^{2+}$ levels prior to the wound induction seemed to be comparable to the wild-type, there was no significant increase in fluorescence intensity in the neighbors, following wound induction, in xit mutants. 


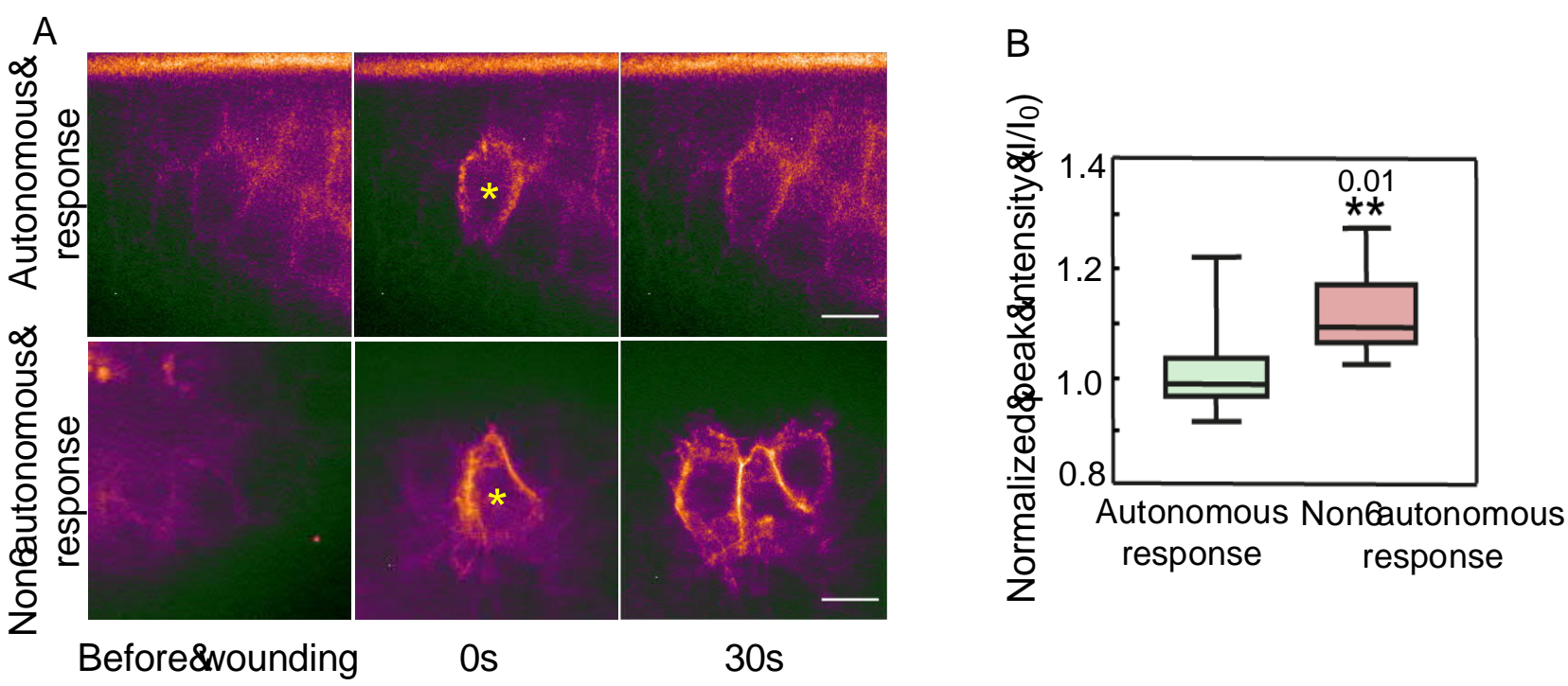

Fig 3.21 $\mathrm{Ca}^{2+}$ dynamics in xit mutant. (A) Post laser cut time points in xit embryos, showing autonomous response (maternal and zygotic depletion) and non-autonomous response (zygotic rescue) to the wounding, in the neighbors. (B) Transient increase in $\mathrm{Ca}^{2+}$ in the neighbors in xit embryos displaying non-autonomous response, compared to the embryos with autonomous response. Boxplot indicate mean \pm SEM. Normalized peak fluorescence intensity was compared in xit embryos, with autonomous (1.010 \pm 0.0267$)$, and nonautonomous $(1.113 \pm 0.02367)$ response to the wounding, at 10 s post laser cut. Statistical significance by Student's T-test ( $p$-value as indicated in the figure). Scale bar $=10 \mu \mathrm{m}$.

\subsection{Increasing extracellular $\mathrm{Mg}^{2+}$ induces morphogenetic defects in AS cells}

I further asked, if the chemical inhibition of $\mathrm{Ca}^{2+}$ dependent ion channel induce an effect on AS cell morphology in a similar manner as loss of TMC. It has been reported in the myocytes from frog ventricle, that $\mathrm{Ca}^{2+}$ dependent ion channels can be blocked rapidly by exposing the cells to the excessive concentration of free $\mathrm{Mg}^{2+}$ ions (Hartzell and White, 1989). Studies in smooth muscle and vascular endothelial cells also suggest that $\mathrm{Mg}^{2+}$ is able to regulate the $\mathrm{Ca}^{2+}$ influx through voltage-gated $\mathrm{Ca}^{2+}$ ion channels (Bara et al., 2001). Excessively present free $\mathrm{Mg}^{2+}$ in the extracellular spaces can bind to and block divalent ion channels, via a competitive inhibitory mechanism.

For the delivery of $\mathrm{Mg}^{2+}$ to the extracellular spaces of the AS cells, the vitelline membrane of the embryos was permeabilized, by treating the embryos with CitraSolve for 2 min (refer to section 2.2.13 for detailed methodology). Embryos were incubated for 10min in 1X PBS (control) and $5-50 \mathrm{mM}$ concentrations of $\mathrm{MgCl}_{2}$. Live time-lapse recording was done to analyze cell morphology. 
A

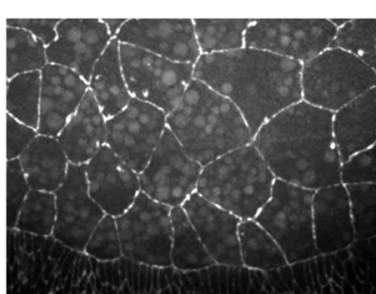

Control (1X PBS)
$\mathrm{MgCl}_{2}$ treated

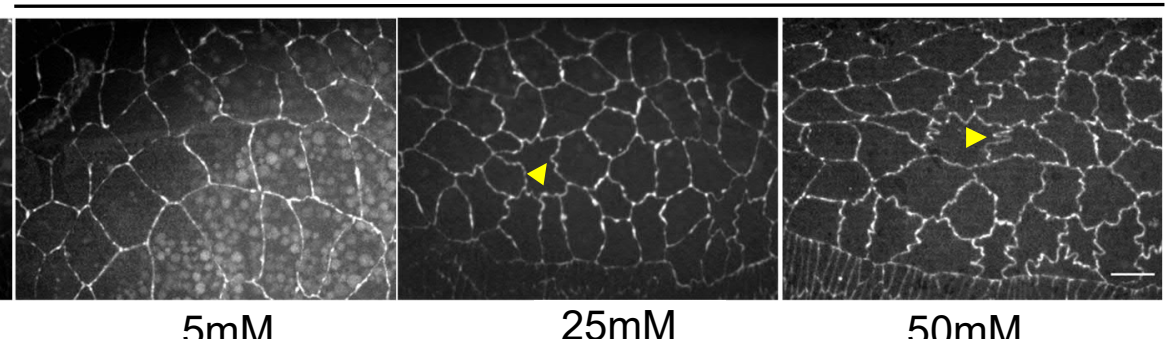

$50 \mathrm{mM}$

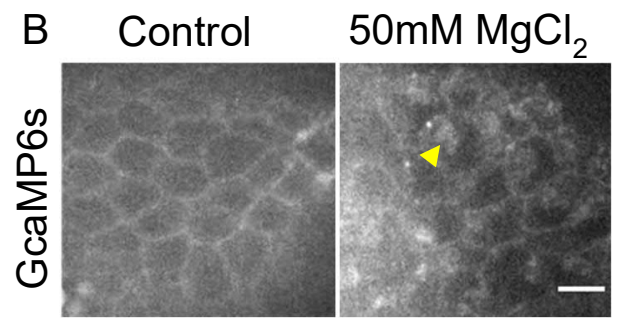

Fig 3.22 $\mathrm{MgCl}_{2}$ treatment induced morphological defects in the AS. (A) Stage 13 embryos incubated in $5 \mathrm{mM}, 25 \mathrm{mM}$ and $50 \mathrm{mM}$ concentration of $\mathrm{MgCl}_{2}$, separately. Treatment of embryos with $\geq 25 \mathrm{mM} \mathrm{MgCl} 2$ for $10 \mathrm{~min}$ resulted in a wavy and undulating morphology of the AS junctions (indicated by the arrow). Permeabilized embryos immersed in 1X PBS were used as control. (B) GcaMP6s expressing wild-type embryos were treated with $50 \mathrm{mM}$ of $\mathrm{MgCl}_{2}$ for $10 \mathrm{~min}$ before the image acquisition. Arrow indicates accumulated GcaMP6s signal at the apical membrane of the embryo. Scale bar $=10 \mu \mathrm{m}$.

Adding $\mathrm{MgCl}_{2}$ in high concentrations $(25 \mathrm{mM}, 50 \mathrm{mM})$ resulted in wavier junctions, compared to straight junctions in control and $5 \mathrm{mM} \mathrm{MgCl}_{2}$ treated embryos (Fig 3.20, A). Furthermore, when wild-type embryos expressing GcaMP6s were treated with high concentrations of $\mathrm{MgCl}_{2}$ (50mM for 10min), accumulated GcaMP6s signal was observed at the apical membrane of the AS cells (Fig 3.20, B, as indicated by the arrow). This accumulated GcaMP6s signal indicated the increased pool of free unbound $\mathrm{Ca}^{2+}$ in the AS cells. The junctional morphological phenotype was similar to the phenotype observed in $T M C^{\Delta}$ mutant embryos. This indicated towards $\mathrm{Ca}^{2+}$ dependent role of TMC in the maintenance of junction morphology. 


\subsection{NOMPC depletion results in overall decrease in junctional tension compared to wild-type}

Since, Phalloidin staining in nompC-RNAi revealed decreased levels of F-actin (Fig 3.3), I investigated the morphology of the AS cells in these embryos. I expressed ECadherin(knock-in) GFP in nompC-RNAi (driven maternally and zygotically). Morphological observation of AS cells in NOMPC depleted embryos revealed wavy junctional morphology similar to $T M C^{\Delta}$ and xit mutants. However, the AS cell shape were isotropic and comparable to the wild-type (Fig 3.23, A).
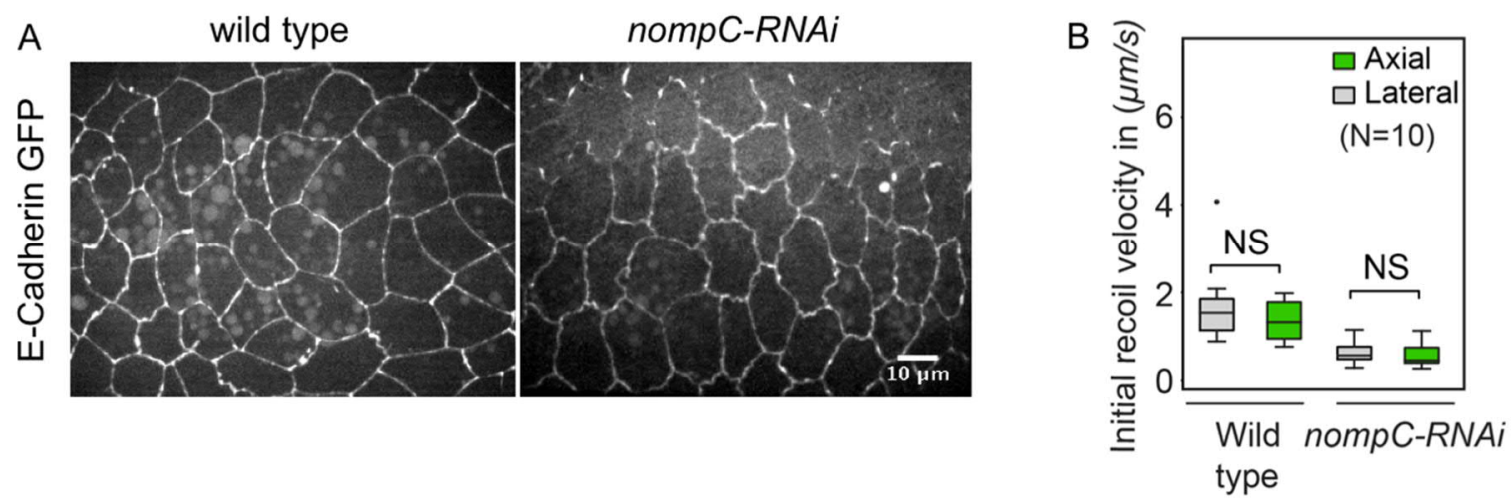

Fig 3.23 NOMPC depletion affects junctional morphology and tension. (A) E-Cadherin expressed in nompC-RNAi embryos, displayed wavy junctional morphology. However, unlike $T M C^{\perp}$ mutants the cell shape remained isotropic and comparable to the wild-type. (B) Boxplot indicate mean and variance of the initial recoil velocity at axially (green) and laterally (grey) oriented AS cell junctions in the wild-type and nompC-RNAi embryos. Laser induced junctional cuts revealed overall decrease in junctional tension (axially as well as laterally oriented junctions) in nompC-RNAi embryos, compared to the wild-type.

In order to reveal tension underlying AS junctions in nompC-RNAi embryos, I did Laser induced junctional ablation. Initial recoil velocity revealed an overall decrease in junctional tension. Additionally, this decrease was independent of the orientation of the junction as both axial as well as lateral orientation displayed decreased recoil velocity and therefore, decreased tension in the nompC-RNAi embryos (Fig 3.23, B). This overall decrease in the junctional tension was consistent with the decrease in F-actin levels in the nompC-RNAi embryos. 


\section{Chapter 4. Discussion}

\subsection{Mechanosensitive ion channels are expressed in the AS epithelial cells in Drosophila}

Mechnosensitive ion channels have been studied extensively in specialized mechanosensory neurons, mainly in the context of sensory neurobiology (Lin and Corey, 2005; Kamikouchi et al., 2009; Effertz et al., 2011; Coste et al., 2012; Guo et al., 2016). However, their function in epithelial cells during early embryonic development has not been explored in detail so far. As reported by genome-wide expression studies (Flybase), MS ion channels are widely expressed in the non-neuronal tissue during embryonic development. In this study, I confirmed the expression of our candidate MS ion channel in the epithelial cells in Drosophila embryo. GFP reporter driven by GAL4, expressed from the promoters of $t m c$, nompC, and piezo, allowed the investigation of their expression in the AS squamous epithelial tissue. Putative MS ion channel TMC was found to be expressed in the amnioserosa tissue, as well as in the epidermis during dorsal-closure. NOMPC, a member of Trp ion channel superfamily was also expressed in the AS and in the epidermis. In case of MS ion channel Piezo, interestingly the GFP reporter expressed via piezo-Gal4 driver displayed a mosaic expression of Piezo in the AS (Fig 3.1). One of the many reasons for this pattern of Piezo expression, could be due to its dependence upon the type of GFP reporter line used with the desired promotor Gal4. In summary, this experiment suggested that MS ion channels are expressed in the epithelium but did not display the actual localization of the ion channel proteins.

\subsubsection{TMC, NOMPC, and Piezo display specific sub-cellular localization in the AS cells}

Immunostaining with specific antibodies, in the AS cells, revealed the sub-cellular localization of the ion channels (TMC and NOMPC). TMC uniformly localizes to the apical and apico-lateral membrane in the AS cells. TMC antibody staining in TMC depleted embryos showed complete loss of protein localization at the apical/apico-medial membrane. Despite the perturbed cell morphology in $T M C^{\Delta}$ mutant AS cells there was no effect on $\mathrm{F}$ actin levels and it was comparable to the wild-type AS cells on the apical as well as on the 
lateral membrane of the cells (Fig 3.2). Apico-lateral localization of TMC indicates a potential overlap with the adherens junctions and E-Cadherin.

NOMPC localized as clusters/punctate structures at the apical and lateral membrane of the AS cells. This localization was strongly reduced in the embryos expressing nompC-RNAi (expressed under control of Maternal-Gal4 driver). The overall F-actin levels were also lowered in nompC-RNAi. Specifically, the apical actin protrusions in the cells were strongly reduced (Fig 3.3). A similar phenotype has been reported upon desensitization of MS ion channels using toxic peptide GsMTx4. GsMTx4 treated embryos show a significant reduction in the number of actin protrusions in the AS cells (Hunter et al., 2014). This phenotype is yet to be reconfirmed in NOMPC null mutants (NOMPC(CRISPR)KO).

Piezo displayed clustered/punctate distribution at the apical as well as apico-lateral membrane. Piezo-GFP positive puncta appeared to dynamically move on the apical/apicomedial surface, similar to Myosin II dynamics reported in the AS cells. This oscillatory accumulation of Piezo puncta/clusters coincided with cell shape change during AS dynamic oscillations. Plausibly, these Piezo foci are linked to the apico-medial actomyosin structures. Thus, the oscillatory accumulation of Piezo could be a passive response to the Myosin II driven oscillations in the AS cells. Piezo ion channels are known to be mechano-gated, and thus able to sense mechanical stretch. Therefore, their involvement in such a dynamically morphogenetic process of AS oscillations cannot be ruled out. However, contrary to our assumption, no morphogenetic changes were observed in the AS cells and dorsal-closure was successfully completed in Piezo-KO embryos. Also, the overall F-actin level was not affected in the Piezo-KO embryos. Further study is required to get more insight into the role of Piezo in dynamically coordinated AS cell behavior.

\subsubsection{Role of TMC and NOMPC during embryogenesis}

Loss of TMC had a mild effect on the embryonic development of the embryos. $18 \%$ of the embryos from the total population did not hatch (tracked until 48h). Further, analyzing the cuticles suggested that among a variety of phenotypes, cuticular holes were dominant and occurred in $70 \%$ of the unhatched embryos. Generally, cuticle holes indicate compromised epithelial cells integrity (Bachmann et al., 2001). The remaining population of the $T M C^{\Delta}$ embryos that hatched successfully were viable and fertile and were kept as homozygous stocks. 
Embryos expressing nompC-RNAi (expressed maternally and zygotically together) showed $50 \%$ embryonic lethality. Among the unhatched population of embryos, approximately $50 \%$ of the embryos displayed cuticle hole phenotype, among other cuticular phenotypes (Fig 3.5). Functional significance of NOMPC during embryonic developmental stages was not assessed further due to the lack of a null mutant. Currently, we have a NOMPC(CRISPR)KO transgene available in the lab which will allow further study of the role of NOMPC.

Unlike $T M C^{\Delta}$ and nompC-RNAi, Piezo-KO mutants were completely viable and fertile and were kept as homozygous stocks. This indicated that Piezo ion-channel might not have an absolutely essential function, for the embryonic development of the Drosophila embryo. However, their dynamic behavior and wide expression suggests that they might contribute to the maintenance of coordination between cells. Further study is required to explore the functional significance of Piezo during embryogenesis.

\subsection{TMC and xit are involved in the regulation of isotropic force balance within the AS}

Probing E-Cadherin in live embryos gave more insight into the role of TMC and xit during the embryonic development. Since the rate of unhatched embryos were low, most of the embryos successfully completed DC. However, I found few embryos that failed to complete the embryonic development and also displayed severe morphological defects in the amnioserosa and epidermis tissue. It is clear that these embryos with severe morphogenetic defects contribute to the of $18 \%$ embryonic lethal population of the $T M C^{\Delta}$ embryos. $T M C^{\Delta}$ mutant embryos which successfully completed dorsal closure were chosen for further analysis to investigate the role of TMC coordinated cell behavior in the AS.

Our group had earlier reported xit-dependent morphogenetic rearrangement of cells via cell intercalation in the lateral epidermis during germ-band extension (Zhang et al., 2014). xit is a glucosyltransferase in the ER (Endoplasmic reticulum) and is involved in $\mathrm{N}$-glycosylation. xit functions indirectly via one or more of its $\mathrm{N}$-glycosylation targets, and $\mathrm{E}$-Cadherin might be one of the major targets of xit during early morphogenetic events. Heterozygous embryos from xit germline clones develop until late embryogenesis and form an AS tissue. During the early DC stage, neighbor cell coordinated non-autonomous AS oscillation is regulated via 
xit dependent pathway. In wild-type embryos, neighboring AS cells stop or slow down their oscillations following laser induced tissue damage. Unlike wild-type, in xit mutant embryos, adjacent cells proceed with area oscillations following junction cuts (according to preliminary experiments done by Dr. Deqing Kong). xit mutants displayed morphogenetic defects in the AS tissue during DC. Since xit functions in an E-Cadherin dependent manner, therefore, cell behavior phenotypes in xit mutant embryos were used as a reference in this study, to which the morphological and behavioral phenotypes of the AS cells in $T M C^{\Delta}$ mutant embryos were compared.

\subsubsection{Maintenance of isotropic morphology of the AS cells during early DC is dependent on TMC and xit}

AS cells in the wild-type embryos display highly anisotropic morphology at the end of germband retraction phase (stage 11-12). These elongated AS cells gradually become isotropic as the germ band retracts and unfolds while the AS tissue is exposed dorsally at the end of stage 13 (Lynch et al., 2013). The transition of AS cells from anisotropic to isotropic morphology, during these developmental stages, was TMC and xit dependent. AS cells in $T M C^{\Delta}$ and xit mutants remained anisotropic and stretched towards the lateral epidermis of the embryo even after the complete retraction of the germband and dorsal ward movement of the AS (Fig 3.8).

Quantification of temporal anisotropy in the AS cells over multiple embryos suggested that majority of the cells in $T M C^{\Delta}$ and xit remained anisotropic post-germ-band retraction. The ability of AS cells to transition from highly anisotropic to isotropic indicate a mechanism within the AS tissue, for the maintenance of the isotropic cell shape, despite anisotropic forces being experienced by the tissue. In wild-type embryos as the DC progresses, contractile forces from the AS and the AC increases. At the same time, there is a contribution of resistive forces from the lateral epidermis surrounding the AS tissue, in order to resist the contractile forces (Hutson et al., 2003). This results in elongated and stretched morphology of epidermal cells at the leading edge. However, despite the presence of anisotropic forces from surrounding, AS cells are able to maintain an isotropic morphology in wild-type embryos. This ability of the AS tissue was lost in $T M C^{\Delta}$ and xit mutants. An explanation for stretched morphology of the AS cells towards lateral epidermis, could be the pronounced forces from the epidermis in $T M C^{\Delta}$ and xit mutants. Continuous pulsatile behavior of AS cells is known to generate tissue scale forces, and contribute to the dorsal ward movement of the lateral epidermis during DC. 
Since the contractile behavior of the AS cells were altered in $T M C^{\Delta}$ and xit mutants (discussed in section 3.5.1 and 4.3.1), it is possible that AS cells failed to efficiently counteract the resistive forces arising from the epidermis, and thus displayed anisotropic morphology. DC still progressed in a normal way in $T M C^{\Delta}$ mutants. Multiple tissue scale coordinated forces contribute to driving the DC (Kiehart et al., 2000a). These highly dynamic processes rely upon complex pathways, which are sufficient to drive the DC in the absence of TMC. Additionally, TMC is one of the many ion channel proteins that are expressed during this stage (For example NOMPC, dTRPA1, Piezo). Therefore, it is less likely that loss of TMC induces severe defects in one of the major morphogenetic processes during embryogenesis. However, the stretched morphology of AS cells did reflect an altered force balance in the tissue.

Interestingly, we also found that increased anisotropy in the AS cells was positiondependent within the tissue, in $T M C^{\Delta}$ and xit mutants. AS cells in the middle were unable to induce isotropic morphology post-germ-band retraction in $T M C^{\Delta}$ and xit mutants. However, AS cells at the periphery stayed comparatively isotropic throughout their transition from germ-band retraction to DC stage. This phenotype was more obvious during the early DC stage. The position-based difference in anisotropy was not as striking in wild-type embryos.

Being a highly morphogenetic event, DC is driven via multiple forces that are intrinsic and extrinsic to the tissue (Kiehart et al., 2000a; Hutson et al., 2003; Solon et al., 2009). AS and epidermis, are the two-main tissue involved in this process, that experience as well as exert forces upon each other during the course of DC. Therefore, despite having the same genotype, it is likely that the cells are influenced from the neighboring tissue (lateral epidermis, AC) to a different extent within the AS, in a position-dependent manner.

\subsubsection{TMC and xit maintain isotropic tension distribution in the AS cells}

Cellular morphology reflects internal and external force/tension being experienced by the cells (Hara and Shagirov et al.,2016). The anisotropic cell shapes in $T M C^{\Delta}$, and xit mutants indicate towards anisotropic tension distribution within the AS, compared to the wild-type. Employing laser-induced cut at the individual AS cell junction using a pulsed UV laser, revealed altered tension distribution within the AS cells in $T M C^{\Delta}$, and xit embryos. In wildtype embryos, tension underlying the AS cell junction remained isotropic, independent of the orientation of the junction within the embryo. Unlike wild-type, AS cells in $T M C^{\Delta}$, and xit had differential tension underlying the junction, based on the orientation of the junction with respect to the anterior-posterior axis of the embryo. In $T M C^{\Delta}$ and xit mutants, two to three- 
fold higher tension was detected underlying the junctions with lateral orientation with respect to the anterior-posterior axis (Fig 3.16). Higher tension in the junctions with lateral orientation was consistent with the laterally stretched anisotropic morphology of the cells in $T M C^{\Delta}$ and xit mutants. Increased recoil velocity in lateral junctions may be due to increased tension or decreased friction in the cells. Interestingly this increase in junctional tension was not observed in axially oriented AS cell junctions in $T M C^{\Delta}$ and xit mutants. The recoil velocities of axially oriented junctions were comparable to the wild-type. This further suggested that increased tension in laterally oriented cell junction might not be as a result of the change in the intrinsic mechanical property of the cells in $T M C^{\Delta}$ and xit mutants, as all the junctions should be affected equally, if that was the case. It has been reported previously that low level of myosin at cell-cell junction lead to wiggly/wavy morphology, suggesting that the wavy cell borders are not under high tension (Blanchard et al., 2010). Surprisingly, despite the wavy junction morphology in $T M C^{\Delta}$ and xit mutants, increased tension was observed only in the laterally oriented junctions. Therefore, anisotropic tension distribution at the lateral AS cell junctions in $T M C^{\Delta}$ and xit could be a result of the external forces being experienced by the AS which is not efficiently counteracted by the tissue.

It is established that the actin cable $(A C)$ at the leading edge is under tension also during the early DC (Hutson et al., 2003). I found that in the wild-type embryos, AC exhibited higher tension at the lateral position compared to the axial position. This finding suggests that anisotropic forces via AC, are exerted on the AS tissue (Fig. 3.17). At the same time, there are resistive forces arising from the epidermis. Given these conditions, it is not surprising that the tension within the tissue is stronger in the lateral, than in the axial direction. However, in wild-type condition these anisotropic forces are counteracted by the contractile behavior of the AS tissue. Loss of TMC and xit revealed the anisotropic forces experienced by the AS tissue. Our findings strongly suggest that TMC and xit are involved in a mechanism that prevents the anisotropy of force distribution in the AS tissue, by evenly spreading the stronger external tension within the tissue, while maintaining a uniform distribution of internal tissue tension and thus morphology (Fig 4.1). 


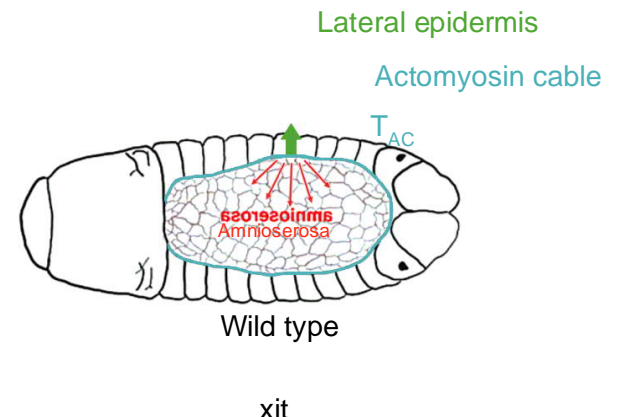

xit

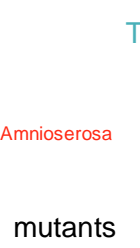

xit

Fig 4.1 TMC and xit play a role in the maintenance of the uniform force distribution within the AS tissue. Lateral tension originating from the lateral epidermis (green) is counteracted by the actomyosin cable (cyan) and AS tissue (red) and is uniformly distributed within the tissue. This is regulated via TMC and xit in wild-type embryos.

\subsection{TMC is an essential component in the pathway establishing coordinated cell behavior in the AS}

Continuous oscillations in the AS cells are non-autonomous and coordinated with the neighbors within the tissue. (Solon et al., 2009; Jayasinghe et al., 2013).TMC ${ }^{\Delta}$, and xit mutant embryos had altered coordinated AS cell behavior. Loss of TMC, and xit affected temporal oscillatory dynamics and intercellular coordination within the cell pairs linked via the junction.

\subsubsection{TMC and xit regulate oscillatory dynamics in AS cells}

The temporal reconstruction of complete AS tissue, with the help of improved segmentation (Cycle-GAN based) approach, allowed the analysis of dynamic cell behavior in the AS. The major period of the cell oscillation from the cell area trajectories were determined via autocorrelation function. The analysis of oscillatory behavior in the AS, revealed faster oscillations in the $T M C^{\Delta}(4.05 \mathrm{~min})$ compared to the wild-type $(5.15 \mathrm{~min})$. Cell oscillations in the xit mutants were faster $\left(2.55 \mathrm{~min}\right.$ ) in comparison to both wild-type and $T M C^{\Delta}$ embryos. These evidences suggest that the dynamic regulation of coordinated oscillatory behavior in the AS is mediated via TMC and xit dependent pathway.

In order to drive such a dynamic cell behavior, a robust regulatory mechanism for force sensing, transduction, and mechanical response would be required within the tissue. 
Generation of forces (mechanical response) in the presence of mechanical stimuli could be driven intrinsically by the cells via the sub-cellular organization of contractile cytoskeletal networks (apico-medial Myosin II pulses) (Martin and Goldstein, 2014; Blanchard et al., 2010; Solon et al., 2009). However, proper sensing of the mechanical stimulus is required in order to trigger a mechanical response. Mechanosensitive ion channels can sense the increased membrane tension in the presence of a mechanical stimulus. Mechanotransduction at the adherens junction of the AS cells, facilitated via TMC, might transduce mechanical stimulus into increased intracellular $\mathrm{Ca}^{2+}$ signal, which further could trigger myosin mediated mechanical response in the cells.

Faster oscillations suggest that the cells are unable to induce efficient cell contractions, and as a result cells start relaxing sooner compared to the wild-type, during AS oscillations. xit functions in an E-Cadherin dependent manner, therefore, the reason for faster oscillations in xit mutants could be a direct result of perturbed interactions of E-Cadherin with actomyosin network.

\subsubsection{TMC mediated mechanotransduction is essential for the distribution of positively coupled cell-pairs within the AS tissue}

In the AS tissue, contact-mediated cell-pairs are temporally coupled during the oscillations. Neighbor dependent contractions in AS cell-pairs can either be positively, negatively, or neutrally correlated in the subpopulation of the cells. Analysis of intercellular coordination of AS cell pairs in wild-type suggested approximately equal distribution of positively and negatively coupled cell pairs in the AS tissue. Interestingly, in case of loss of TMC, this balance shifted with a significant decrease in the population of positively coupled cells types, while the overall correlation remained unaffected. These findings suggest TMC dependent distribution of positive coupling of cell pairs within the AS tissue. It is possible that TMC selectively mediates mechanotransduction at the junction between positively coupled cell pairs. In contrast, all aspects of correlation were affected in xit depleted embryos, including a strong reduction in the total number of correlated cell pairs (negatively and positively coupled) (Fig 3.13).

These results confirmed that there is a contact-based coupling of AS cell pairs in order to drive dynamic oscillations (Jayasinghe et al., 2013; Wang et al., 2012). Further, TMCmediated mechano-transduction in the AS is essential for maintaining even distribution of 
both coupling types. However, the overall strength of the correlation is not TMC dependent. A key aspect of the stationary phase of oscillation in the AS, is that the total area of the AS tissue remains unchanged, despite continuous fluctuations in the AS cells. In wild-type embryos, this property is maintained by keeping an equal distribution of positive and negative correlated cell pairs, during AS oscillations, in TMC dependent manner.

It is known that actomyosin driven cell contractions can be induced in AS cells in a $\mathrm{Ca}^{2+}$ dependent manner. Therefore, the requirement of $\mathrm{Ca}^{2+}$ signaling and dynamics is higher in positively correlated cell pairs, as they induce contraction together at the same time. However, in negatively correlated cell pairs only one of the two cells induce contractions at a time and thus, there is a limited requirement of $\mathrm{Ca}^{2+}$. I found that $\mathrm{Ca}^{2+}$ signaling dependent response in the neighbors was significantly reduced in the absence of TMC (section 4.4). This may provide an explanation for the selective reduction in positively coupled cells in $T M C^{\Delta}$ mutant embryos. Positively coupled cells require additional mechanotransduction at the linked junctions and in AS cells, this requirement is probably fulfilled via TMC mediated pathway (Fig 4.2). Also, cell oscillations were faster in $T M C^{\Delta}$ mutant embryos suggesting perturbed contractile behavior of the AS cells. This further point towards perturbed $\mathrm{Ca}^{2+}$ dynamics and transduction in the AS cells, during oscillations in $T M C^{\Delta}$ mutant.

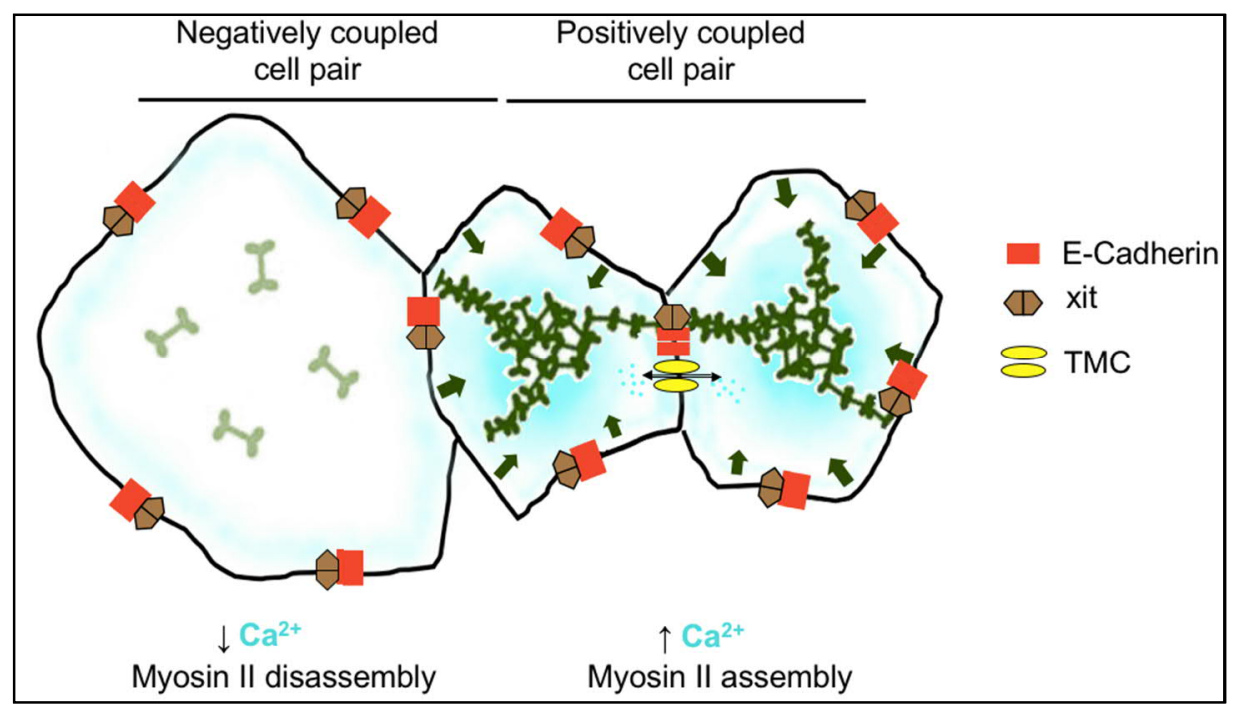

Fig 4.2 TMC and xit dependent mechanotransduction distinctly control the coordination of the coupled cell pairs. TMC is required for mechanotransduction between positively coupled AS cell-pairs and xit controls overall coordination. TMC functions in $\mathrm{Ca}^{2+}$ dependent manner and thus, fulfills the increased requirement of $\mathrm{Ca}^{2+}$ signaling in positively coupled cell-pair during contractions. xit mediated mechanotransduction is required for overall coordination, thus, xit might function in an E-Cadherin dependent manner at the junction of all types of correlated cell pair. 
xit dependent mechano-transduction is essential for overall coordination between the neighboring cells as all the aspects of correlation were affected in xit mutants, independent of the coupling type of the cells. It is likely that xit is required at each junction irrespective of the correlation type of the cell pairs and thus, affects overall neighbor cell interaction and coordination in an E-Cadherin dependent manner.

\subsection{TMC play a role in $\mathrm{Ca}^{2+}$ dependent response of the neighboring cells to wounding}

In the AS cells, immediate $\mathrm{Ca}^{2+}$ influx is triggered in the neighboring cells as a response to wounding or tissue damage. In $T M C^{\Delta}$ mutants, there was a significant decrease in $\mathrm{Ca}^{2+}$ influx in the neighbors. This suggested that TMC is involved directly or indirectly in signal transduction, to trigger wound response in the neighbors. However, a complete loss of $\mathrm{Ca}^{2+}$ dependent response was not observed in the $T M C^{\Delta}$ mutants. It is not surprising, considering the fact that other major $\mathrm{Ca}^{2+}$ dependent ion channels are also expressed during these stages (NOMPC, dTRPA1, Piezo) and could compensate for the loss of TMC function. These experiments provide strong evidence for $\mathrm{Ca}^{2+}$ dependent function of TMC as it had a direct effect on $\mathrm{ca}^{2+}$ signaling dynamics in the AS cells.

In xit mutants, there was a significant decrease in $\mathrm{Ca}^{2+}$ dependent neighbor cell response. Despite of the transient increase in $\mathrm{Ca}^{2+}$ response in the neighbors in approximately half of the xit germline clone embryos, total $\mathrm{Ca}^{2+}$ dependent response was significantly reduced independent of the genotype. In addition to this, the overall coordination between cell pairs was also significantly reduced within the AS in xit mutant embryos. Based on these findings, it is likely that as a result of lack of coordination between the AS cells, the change in mechanical properties following wounding of the target cell is not being transduced to the neighbors in xit mutant embryos.

Further, chemical inhibition of $\mathrm{Ca}^{2+}$ dependent ion-channels induced similar morphological phenotype in the AS cells as the loss of TMC. To specifically block the $\mathrm{Ca}^{2+}$ dependent ionchannels expressed at this stage of development, the concentration of free $\mathrm{Mg}^{2+}$ was excessively increased in the extracellular spaces. This excessively present $\mathrm{Mg}^{2+}$ can bind to and block divalent ion channels, via a competitive inhibitory mechanism (Hartzell and White, 1989). Wavy junctional morphology in the AS cells was observed upon chemical inhibition 
of $\mathrm{Ca}^{2+}$ dependent ion channels. This phenotype was similar to the junctional morphological phenotype in $T M C^{\Delta}$ mutant. The similarity in phenotype further emphasized that TMC might functions as a $\mathrm{Ca}^{2+}$ dependent MS ion channel in the AS cells. (Fig 3.21).

In auditory hair cell system, mutations of TMC1 alters the conductance and $\mathrm{Ca}^{2+}$ selectivity of the MET channels (cation channel with high selectivity for $\mathrm{Ca}^{2+}$ ) (Kurima et al., 2002; 2003; Kim and Fettiplace, 2013). However, whether TMC itself is involved in $\mathrm{Ca}^{2+}$ dependent transduction remained elusive so far. This study suggested that putative ion channel TMC is involved and contribute to the $\mathrm{Ca}^{2+}$ dependent mechanotransduction in the epithelial cells.

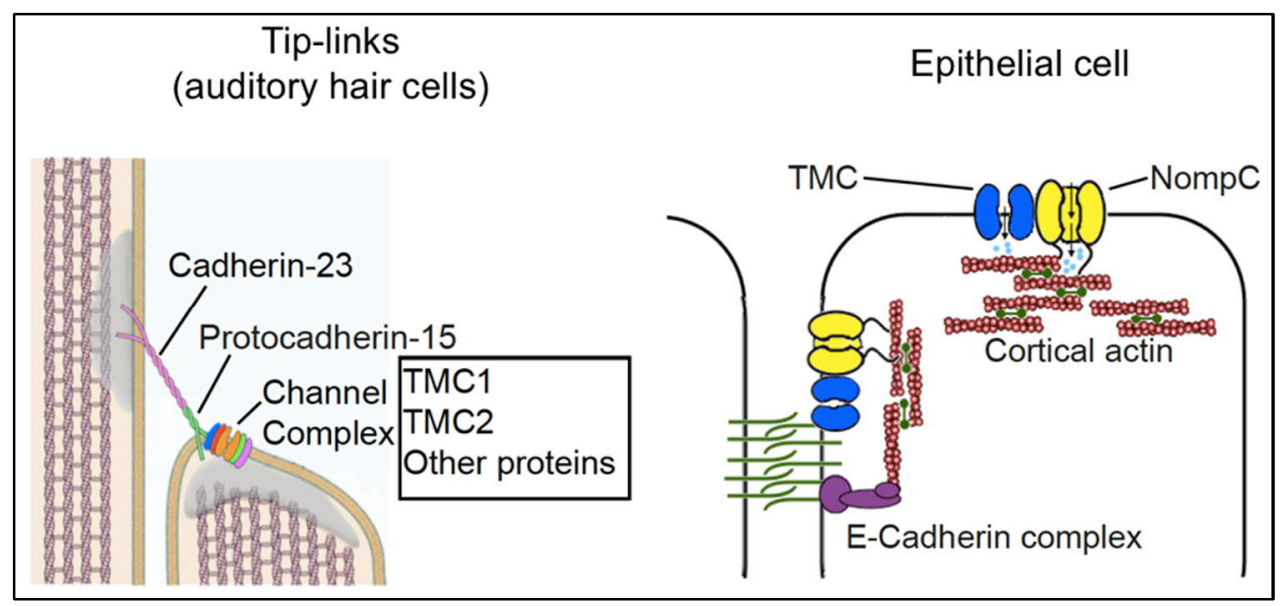

Fig 4.3 Insight from functional mechanism of TMC in the auditory system. In the inner ear hair cells of the auditory system, protocadherin (sensing the differential movement of hair cells), are linked to the channel complex, including TMC and MET ion channel (schematic drawing according to Kurima, 2015). In the epithelial cells, we hypothesize that TMC (probably with other ion channels) is linked either directly to the E-Cadherin complex at the adherens junctions or indirectly via cortical actin.

TMC is well studied in extracellular protein filaments known as 'tip links' that are required for mechanotransduction in auditory hair cells (Hudspeth, 1989). At the tip links, TMC has been shown to interact with the transmembrane and cytoplasmic domains of PCDH15. Interaction of TMC with E-Cadherin in epithelial cells could not be ruled out based on the range of phenotype affecting coordination between cell pairs linked via junction in the AS. Additionally, the similarity of phenotypes in TMC and xit mutants also indicate towards the junctional association of TMC (Fig 4.3). In order to determine the physical interaction of TMC with E-Cadherin in the epithelial cells, I will perform Co-Immunoprecipitation experiment in wild-type embryos. 
At the tip-links of auditory hair cells, TMC is known to functions in association with the large ion-channel complexes (MET, TRP) (Kurima et al., 2015). Such an association of TMC is also likely in the epithelial cells. In epithelial cells, one of the candidates for TMC association could be NOMPC, a $\mathrm{Ca}^{2+}$ dependent ion channel, which is a member of the transient receptor potential (TRP) family of ion channels. NOMPC depletion in embryos induced junctional morphological phenotypes similar to $T M C^{\Delta}$ and xit mutants. However, unlike $T M C^{\Delta}$ and xit embryos, recoil velocity at the AS cell junctions was decreased in nompC$R N A i$ embryos, independent of orientation of the junction with respect to the anteriorposterior axis of the embryo. Decrease in F-actin levels in the AS cells in nompC-RNAi mutants, was consistent with decrease in the junctional tension. It is likely that mechanotransduction via NOMPC ion-channel can regulate actomyosin networks in the AS tissue, in a $\mathrm{Ca}^{2+}$ dependent manner. Such a functional mechanism for TRP ion-channels has been suggested in the Drosophila photoreceptor cells. Light exposure mediated opening of TRP channels can actively redistribute and reshape the actin cytoskeleton (Kosloff et al., 2003) (Fig 4.3).

In this study, I was able to establish that the putative ion channel TMC is expressed and play a role in regulation of coordinated cell behavior in the epithelial cells in Drosophila embryos. TMC is an essential component for the dynamic regulation of coordinated cell behavior in the AS cells. TMC might provide a molecular link between adherens junctions and cytoskeletal networks, and is able to transduce mechanical force into increased intracellular $\mathrm{Ca}^{2+}$. Specifically, TMC dependent mechanotransduction is crucial for the efficient positive coupling of the AS cell pairs and thus, maintain even distribution of both positively and negatively coupled cell types in the wild-type embryos. TMC also play a role in the regulation of force-induced cell behavior of the AS cells, which further maintains isotropic distribution of tissue tension and thus, isotropic cellular morphology in the AS. 


\section{References}

Abreu-Blanco, M. T., Verboon, J. M., Liu, R., Watts, J. J., and Parkhurst, S. M. (2012). Drosophila embryos close epithelial wounds using a combination of cellular protrusions and an actomyosin purse string. Journal of Cell Science 125, 5984-5997.

Andrew, D. J., and Ewald, A. J. (2010). Morphogenesis of epithelial tubes: Insights into tube formation, elongation, and elaboration. Developmental Biology 341, 34-55.

Antunes, M., Pereira, T., Cordeiro, J. V., Almeida, L., and Jacinto, A. (2013). Coordinated waves of actomyosin flow and apical cell constriction immediately after wounding. J. Cell Biol. 202, 365-379.

Árnadóttir, J., and Chalfie, M. (2010). Eukaryotic Mechanosensitive Channels. Annu. Rev. Biophys. 39, 111-137.

Bagur, R., and Hajnóczky, G. (2017). Intracellular Ca2+ Sensing: Its Role in Calcium Homeostasis and Signaling. Molecular Cell 66, 780-788.

Bachmann, A., Schneider, M., Theilenberg, E., Grawe, F., and Knust, E. (2001). Drosophila Stardust is a partner of Crumbs in the control of epithelial cell polarity. Nature $2017541: 7637$ 414, 638-643.

Bara, M., and Guiet-Bara, A. (2001). Magnesium regulation of $\mathrm{Ca}^{2+}$ channels in smooth muscle and endothelial cells of human allantochorial placental vessels. Magnes Res 14, $11-18$.

BERSHADSKY, A., Ballestrem, C., CARRAMUSA, L., ZILBERMAN, Y., GILQUIN, B., KHOCHBIN, S., ALEXANDROVA, A., VERKHOVSKY, A., SHEMESH, T., and KOZLOV, M. (2006). Assembly and mechanosensory function of focal adhesions: experiments and models. European Journal of Cell Biology 85, 165-173.

Beurg, M., Xiong, W., Zhao, B., Müller, U., and Fettiplace, R. (2015). Subunit determination of the conductance of hair-cell mechanotransducer channels. Pnas 112, 1589-1594. 
Blanchard, G. B., Murugesu, S., Adams, R. J., Martinez-Arias, A., and Gorfinkiel, N. (2010). Cytoskeletal dynamics and supracellular organisation of cell shape fluctuations during dorsal closure. Development 137, 2743-2752.

Borghi, N., Sorokina, M., Shcherbakova, O. G., Weis, W. I., Pruitt, B. L., Nelson, W. J., and Dunn, A. R. (2012). E-cadherin is under constitutive actomyosin-generated tension that is increased at cell-cell contacts upon externally applied stretch. Pnas $109,12568-$ 12573.

Brand, A. H., and Perrimon, N. (1993). Targeted gene expression as a means of altering cell fates and generating dominant phenotypes. Development 118, 401-415.

Campos-Ortega, J. A., and Hartenstein, V. (1997). Stages of Drosophila Embryogenesis. In: The Embryonic Development of Drosophila Melanogaster, Berlin, Heidelberg: Springer, Berlin, Heidelberg, 9-102.

Cavey, M., Rauzi, M., Lenne, P.-F., and Lecuit, T. (2008). A two-tiered mechanism for stabilization and immobilization of E-cadherin. Nature 2017 541:7637 453, 751-756.

Chanet, S., and Martin, A. C. (2014). Mechanical Force Sensing in Tissues. Progress in Molecular Biology and Translational Science 126, 317-352.

Christensen, A. P., and Corey, D. P. (2007). TRP channels in mechanosensation: direct or indirect activation? Nature Reviews Neuroscience 2007 8:7 8, 510-521.

Chou, T. B., and Perrimon, N. (1992). Use of a yeast site-specific recombinase to produce female germline chimeras in Drosophila. Genetics 131, 643-653.

Clapham, D. E., Runnels, L. W., and Strübing, C. (2001). The trp ion channel family. Nature Reviews Neuroscience 2007 8:7 2, 387-396.

Clark, K., Middelbeek, J., and VANLEEUWEN, F. (2008). Interplay between TRP channels and the cytoskeleton in health and disease. European Journal of Cell Biology 87, 631-640.

Coste, B. et al. (2012). Piezo proteins are pore-forming subunits of mechanically activated channels. Nature $2017541: 7637$ 483, 176-181. 
Dawes-Hoang, R. E., Parmar, K. M., Christiansen, A. E., Phelps, C. B., Brand, A. H., and Wieschaus, E. F. (2005). folded gastrulation, cell shape change and the control of myosin localization. Development 132, 4165-4178.

Diz-Muñoz, A., Fletcher, D. A., and Weiner, O. D. (2013). Use the force: membrane tension as an organizer of cell shape and motility. Trends Cell Biol. 23, 47-53.

Drees, F., Pokutta, S., Yamada, S., Nelson, W. J., and Weis, W. I. (2005). a-Catenin Is a Molecular Switch that Binds E-Cadherin- $\beta$-Catenin and Regulates Actin-Filament Assembly. Cell 123, 903-915.

Ducuing, A., and Vincent, S. (2016). The actin cable is dispensable in directing dorsal closure dynamics but neutralizes mechanical stress to prevent scarring in the Drosophila embryo. Nat. Cell Biol. 18, 1149-1160.

Effertz, T., Wiek, R., and Göpfert, M. C. (2011). NompC TRP Channel Is Essential for Drosophila Sound Receptor Function. Current Biology 21, 592-597.

Enyedi, B., and Niethammer, P. (2015). Mechanisms of epithelial wound detection. Trends Cell Biol. 25, 398-407.

Etournay, R., Merkel, M., Popović, M., Brandl, H., Elife, N. D., 2016 TissueMiner: a multiscale analysis toolkit to quantify how cellular processes create tissue dynamics. Cdn.Elifesciences.org

Fettiplace, R. (2016). Is TMC1 the Hair Cell Mechanotransducer Channel? Biophysical Journal 111, 3-9.

Gates, J., and Peifer, M. (2005). Can 1000 Reviews Be Wrong? Actin, $\alpha-C a t e n i n$, and Adherens Junctions. Cell 123, 769-772.

Gilmour, D., Rembold, M., and Leptin, M. (2017). From morphogen to morphogenesis and back. Nature $2017541: 7637$ 541, 311-320.

Göpfert, M. C., Albert, J. T., Nadrowski, B., and Kamikouchi, A. (2006). Specification of auditory sensitivity by Drosophila TRP channels. Nature Neuroscience 2006 9:8 9, 9991000. 
Gudipaty, S. A., Lindblom, J., Loftus, P. D., Redd, M. J., Edes, K., Davey, C. F., Krishnegowda, V., and Rosenblatt, J. (2017). Mechanical stretch triggers rapid epithelial cell division through Piezo1. Nature $2017541: 7637$ 543, 118-121.

Guillot, C., and Lecuit, T. (2013). Mechanics of Epithelial Tissue Homeostasis and Morphogenesis. Science 340, 1185-1189.

Gumbiner, B. M. (1996). Cell Adhesion: The Molecular Basis of Tissue Architecture and Morphogenesis. Cell 84, 345-357.

Guo, Y. et al. (2016). Transmembrane channel-like $(\mathrm{tmc})$ gene regulates Drosophila larval locomotion. Pnas 113, 7243-7248.

Haering, M., Grosshans, J., Wolf, F., and Eule, S. (2018). Automated Segmentation of Epithelial Tissue Using Cycle-Consistent Generative Adversarial Networks. bioRxiv, 311373.

Hamada, F. N., Rosenzweig, M., Kang, K., Pulver, S. R., Ghezzi, A., Jegla, T. J., and Garrity, P. A. (2008). An internal thermal sensor controlling temperature preference in Drosophila. Nature 2017 541:7637 454, 217-220.

Harris, T. J. C., and Tepass, U. (2010). Adherens junctions: from molecules to morphogenesis. 11, 502-514.

Hartzell, H. C., and White, R. E. (1989). Effects of magnesium on inactivation of the voltage-gated calcium current in cardiac myocytes. The Journal of General Physiology $94,745-767$.

He, L., Si, G., Huang, J., Samuel, A. D. T., and Perrimon, N. (2018). Mechanical regulation of stem-cell differentiation by the stretch-activated Piezo channel. Nature 2017 541:7637 555, 103-106.

He, L., Wang, X., Tang, H. L., and Montell, D. J. (2010). Tissue elongation requires oscillating contractions of a basal actomyosin network. Nat. Cell Biol. 12, 1133-1142.

Heer, N. C., and Martin, A. C. (2017). Tension, contraction and tissue morphogenesis. Development 144, 4249-4260. 
Heisenberg, C.P. (2009). Dorsal closure in Drosophila: cells cannot get out of the tight spot. BioEssays 31, 1284-1287.

Heisenberg, C.-P., and Bellaïche, Y. (2013). Forces in Tissue Morphogenesis and Patterning. Cell 153, 948-962.

Hudspeth, A. J. (1989). How the ear's works work. Nature $2017541: 7637$ 341, 397-404.

Hunter, G. L., Crawford, J. M., Genkins, J. Z., and Kiehart, D. P. (2014). Ion channels contribute to the regulation of cell sheet forces during Drosophila dorsal closure. Development 141, 325-334.

Hutson, M. S., Tokutake, Y., Chang, M.-S., Bloor, J. W., Venakides, S., Kiehart, D. P., and Edwards, G. S. (2003). Forces for Morphogenesis Investigated with Laser Microsurgery and Quantitative Modeling. Science 300, 145-149.

Hu, Y., Wang, Z., Liu, T., and Zhang, W. (2019). Piezo-like Gene Regulates Locomotion in Drosophila Larvae. Cell Reports 26, 1369-1377.e4.

Jacinto, A., Woolner, S., and Martin, P. (2002). Dynamic Analysis of Dorsal Closure in Drosophila: From Genetics to Cell Biology. Developmental Cell 3, 9-19.

Jayasinghe, A. K., Crews, S. M., Mashburn, D. N., and Hutson, M. S. (2013). Apical Oscillations in Amnioserosa Cells: Basolateral Coupling and Mechanical Autonomy. Biophysical Journal 105, 255-265.

Jin, P. et al. (2017). Electron cryo-microscopy structure of the mechanotransduction channel NOMPC. Nature 2017 541:7637 547, 118-122.

Kamikouchi, A., Inagaki, H. K., Effertz, T., Hendrich, O., Fiala, A., Göpfert, M. C., and Ito, K. (2009). The neural basis of $<i>$ Drosophila $</ i>$ gravity-sensing and hearing. Nature $2017541: 7637458,165-171$.

Kaneuchi, T., Sartain, C. V., Takeo, S., Horner, V. L., Buehner, N. A., Aigaki, T., and Wolfner, M. F. (2015). Calcium waves occur as Drosophila oocytes activate. Pnas 112, 791-796.

Keller, R. (2012). Physical Biology Returns to Morphogenesis. Science 338, 201-203. 
Kiehart, D. P., Galbraith, C. G., Edwards, K. A., Rickoll, W. L., and Montague, R. A. (2000a). Multiple Forces Contribute to Cell Sheet Morphogenesis for Dorsal Closure in Drosophila. J. Cell Biol. 149, 471-490.

Kiehart, D. P., Galbraith, C. G., Edwards, K. A., Rickoll, W. L., and Montague, R. A. (2000b). Multiple forces contribute to cell sheet morphogenesis for dorsal closure in Drosophila. J. Cell Biol. 149, 471-490.

Kim, K. X., and Fettiplace, R. (2013). Developmental changes in the cochlear hair cell mechanotransducer channel and their regulation by transmembrane channel-like proteins. The Journal of General Physiology 141, 141-148.

Kim, S. E., Coste, B., Chadha, A., Cook, B., and Patapoutian, A. (2012). The role of Drosophila Piezo in mechanical nociception. Nature 2017 541:7637 483, 209-212.

Kitazawa, T., Masuo, M., and Somlyo, A. P. (1991). G protein-mediated inhibition of myosin light-chain phosphatase in vascular smooth muscle. Pnas 88, 9307-9310.

Kong, D., Lv, Z., Häring, M., Wolf, F., and Grosshans, J. (2019). In vivo optochemical control of cell contractility at single cell resolution by $\mathrm{Ca} 2+$ induced myosin activation. bioRxiv, 255372.

Kong, D., Wolf, F., and Großhans, J. (2017). Forces directing germ-band extension in Drosophila embryos. Mechanisms of Development 144, 11-22.

Kosloff, M., Elia, N., Almagor, T. J., Timberg, R., Zars, T. D., Hyde, D. R., Minke, B., and Selinger, Z. (2003). Regulation of light-dependent Gqa translocation and morphological changes in fly photoreceptors. The EMBO Journal 22, 459-468.

Kölsch, V., Seher, T., Fernandez-Ballester, G. J., Serrano, L., and Leptin, M. (2007). Control of Drosophila Gastrulation by Apical Localization of Adherens Junctions and RhoGEF2. Science 315, 384-386.

Kurima, K. et al. (2002). Dominant and recessive deafness caused by mutations of a novel gene, TMC1, required for cochlear hair-cell function. Nat Genet 30, 277-284.

Kurima, K. et al. (2015). TMC1 and TMC2 Localize at the Site of Mechanotransduction in Mammalian Inner Ear Hair Cell Stereocilia. Cell Reports 12, 1606-1617. 
Kurima, K., Yang, Y., Sorber, K., and Griffith, A. J. (2003). Characterization of the transmembrane channel-like (TMC) gene family: functional clues from hearing loss and epidermodysplasia verruciformis 败. Genomics 82, 300-308.

Lee, H. C., and Auersperg, N. (1980). Calcium in epithelial cell contraction. J. Cell Biol. 85, 325-336.

Leptin, M., and Grunewald, B. (1990). Cell shape changes during gastrulation in Drosophila. Development 110, 73-84.

Lin, S.-Y., and Corey, D. P. (2005). TRP channels in mechanosensation. Current Opinion in Neurobiology 15, 350-357.

Liang, X., Madrid, J., Saleh, H. S., and Howard, J. (2011). NOMPC, a member of the TRP channel family, localizes to the tubular body and distal cilium of Drosophila campaniform and chordotonal receptor cells. Cytoskeleton 68, 1-7.

Lynch, H. E., Crews, S. M., Rosenthal, B., Kim, E., Gish, R., Echiverri, K., and Hutson, M. S. (2013). Cellular mechanics of germ band retraction in Drosophila. Developmental Biology 384, 205-213.

Maeda, R., Kindt, K. S., Mo, W., Morgan, C. P., Erickson, T., Zhao, H., ClemensGrisham, R., Barr-Gillespie, P. G., and Nicolson, T. (2014). Tip-link protein protocadherin 15 interacts with transmembrane channel-like proteins TMC1 and TMC2. Pnas 111, 12907-12912.

Mahmud, A. A. et al. (2017). Loss of the proprioception and touch sensation channel PIEZO2 in siblings with a progressive form of contractures. Clinical Genetics 91, 470475.

Maitre, J.L., and Heisenberg, C.P. (2013). Three Functions of Cadherins in Cell Adhesion. Current Biology 23, R626-R633.

Markova, O., Senatore, S., and Lenne, P.-F. (2019). Spatiotemporal dynamics of calcium transients during embryogenesis of Drosophila melanogaster. bioRxiv, 540070 .

Martin, A. C., and Goldstein, B. (2014). Apical constriction: themes and variations on a cellular mechanism driving morphogenesis. Development 141, 1987-1998. 
Mateus, A. M., and Arias, A. M. (2011). Patterned Cell Adhesion Associated with Tissue Deformations during Dorsal Closure in Drosophila. Plos One 6, e27159.

Matsuzawa, K., Himoto, T., Mochizuki, Y., and Ikenouchi, J. (2018). a-Catenin Controls the Anisotropy of Force Distribution at Cell-Cell Junctions during Collective Cell Migration. Cell Reports 23, 3447-3456.

Niessen, C. M., Leckband, D., and Yap, A. S. (2011). Tissue Organization by Cadherin Adhesion Molecules: Dynamic Molecular and Cellular Mechanisms of Morphogenetic Regulation. Physiological Reviews 91, 691-731.

Orr, A. W., Helmke, B. P., Blackman, B. R., and Schwartz, M. A. (2006). Mechanisms of Mechanotransduction. Developmental Cell 10, 11-20.

Oda, H., and Tsukita, S. (2001). Real-time imaging of cell-cell adherens junctions reveals that Drosophila mesoderm invagination begins with two phases of apical constriction of cells. Journal of Cell Science 114, 493-501.

Pan, B. et al. (2018). TMC1 Forms the Pore of Mechanosensory Transduction Channels in Vertebrate Inner Ear Hair Cells. Neuron 99, 736-753.e736.

Pan, B., Géléoc, G. S., Asai, Y., Horwitz, G. C., Kurima, K., Ishikawa, K., Kawashima, Y., Griffith, A. J., and Holt, J. R. (2013). TMC1 and TMC2 Are Components of the Mechanotransduction Channel in Hair Cells of the Mammalian Inner Ear. Neuron 79, 504-515.

Pasakarnis, L., Frei, E., Caussinus, E., Affolter, M., and Brunner, D. (2016). Amnioserosa cell constriction but not epidermal actin cable tension autonomously drives dorsal closure. Nat. Cell Biol. 18, 1161-1172.

Martin, P., and Lewis, J. (1992). Actin cables and epidermal movement in embryonic wound healing. Nature $2017541: 7637$ 360, 179-183.

Quintin, S., Gally, C., and Labouesse, M. (2008). Epithelial morphogenesis in embryos: asymmetries, motors and brakes. Trends in Genetics 24, 221-230.

Ranade, S. S. et al. (2014). Piezo1, a mechanically activated ion channel, is required for vascular development in mice. Pnas 111, 10347-10352. 
Rand, M. D., Kearney, A. L., Dao, J., and Clason, T. (2010). Permeabilization of Drosophila embryos for introduction of small molecules. Insect Biochemistry and Molecular Biology 40, 792-804.

Restrepo, S., and Basler, K. (2016). Drosophila wing imaginal discs respond to mechanical injury via slow InsP3R-mediated intercellular calcium waves. Nature Communications 20167 7, 12450.

Rohrschneider, M. R., and Nance, J. (2009). Polarity and cell fate specification in the control of Caenorhabditis elegans gastrulation. Developmental Dynamics 238, 789-796.

Ronneberger, O., Fischer, P., and Brox, T. (2015). U-Net: Convolutional Networks for Biomedical Image Segmentation. In: Medical Image Computing and Computer-Assisted Intervention - MICCAI 2015, Cham: Springer, Cham, 234-241.

Sammak, P. J., Hinman, L. E., Tran, P. O., Sjaastad, M. D., and Machen, T. E. (1997). How do injured cells communicate with the surviving cell monolayer? Journal of Cell Science 110, 465-475.

Saotome, K., Murthy, S. E., Kefauver, J. M., Whitwam, T., Patapoutian, A., and Ward, A. B. (2018). Structure of the mechanically activated ion channel Piezo1. Nature 2017 $541: 7637554,481-486$.

Sawyer, J. M., Harrell, J. R., Shemer, G., Sullivan-Brown, J., Roh-Johnson, M., and Goldstein, B. (2010). Apical constriction: A cell shape change that can drive morphogenesis. Developmental Biology 341, 5-19.

Shannon, E. K., Stevens, A., Edrington, W., Zhao, Y., Jayasinghe, A. K., Page-McCaw, A., and Hutson, M. S. (2017). Multiple Mechanisms Drive Calcium Signal Dynamics around Laser-Induced Epithelial Wounds. Biophysical Journal 113, 1623-1635.

Sokolow, A., Toyama, Y., Kiehart, D. P., and Edwards, G. S. (2012). Cell Ingression and Apical Shape Oscillations during Dorsal Closure in Drosophila. Biophysical Journal 102, 969-979.

Solon, J., Kaya-Çopur, A., Colombelli, J., and Brunner, D. (2009). Pulsed Forces Timed by a Ratchet-like Mechanism Drive Directed Tissue Movement during Dorsal Closure. Cell $137,1331-1342$. 
Sullivan, K. M. C., Scott, K., Zuker, C. S., and Rubin, G. M. (2000). The ryanodine receptor is essential for larval development in Drosophila melanogaster. Pnas 97, 59425947.

Suzuki, M. et al. (2017). Distinct intracellular Ca2+ dynamics regulate apical constriction and differentially contribute to neural tube closure. Development 144, 1307-1316.

Toyama, Y., Peralta, X. G., Wells, A. R., Kiehart, D. P., and Edwards, G. S. (2008). Apoptotic Force and Tissue Dynamics During Drosophila Embryogenesis. Science 321, 1683-1686.

Truong Quang, B.-A., Mani, M., Markova, O., Lecuit, T., and Lenne, P.-F. (2013). Principles of E-Cadherin Supramolecular Organization In Vivo. Current Biology 23, 2197-2207.

Twiss, F., Le Duc, Q., Van Der Horst, S., Tabdili, H., Van Der Krogt, G., Wang, N., Rehmann, H., Huveneers, S., Leckband, D. E., and De Rooij, J. (2012). Vinculindependent Cadherin mechanosensing regulates efficient epithelial barrier formation. Biology Open 1, 1128-1140.

Vasquez, C. G., Tworoger, M., and Martin, A. C. (2014). Dynamic myosin phosphorylation regulates contractile pulses and tissue integrity during epithelial morphogenesis. J. Cell Biol. 206, 435-450.

Walker, R. G., Willingham, A. T., and Zuker, C. S. (2000). A Drosophila Mechanosensory Transduction Channel. Science 287, 2229-2234.

Wood, W. (2012). Wound Healing: Calcium Flashes Illuminate Early Events. Current Biology 22, R14-R16.

Yan, Z., Zhang, W., He, Y., Gorczyca, D., Xiang, Y., Cheng, L. E., Meltzer, S., Jan, L. Y., and Jan, Y. N. (2013). Drosophila NOMPC is a mechanotransduction channel subunit for gentle-touch sensation. Nature 2017 541:7637 493, 221-225.

Yonemura, S., Wada, Y., Watanabe, T., Nagafuchi, A., and Shibata, M. (2010). aCatenin as a tension transducer that induces adherens junction development. Nat. Cell Biol. 12, 533-542. 
Zhang, W. et al. (2015). Ankyrin Repeats Convey Force to Gate the NOMPC Mechanotransduction Channel. Cell 162, 1391-1403.

Zhang, Y. V., Aikin, T. J., Li, Z., and Montell, C. (2016). The Basis of Food Texture Sensation in Drosophila. Neuron 91, 863-877.

Zhang, Y., Kong, D., Reichl, L., Vogt, N., Wolf, F., and Großhans, J. (2014). The glucosyltransferase Xiantuan of the endoplasmic reticulum specifically affects ECadherin expression and is required for gastrulation movements in Drosophila. Developmental Biology 390, 208-220. 


\section{Appendix}

Index for appendix:

Title

Description

Page No.

Appendix 1: Anisotropy for AS cells at the periphery. Anisotropy and cell angle plotted for single cases.

Appendix 2: Displacement trajectories for junctions following UV laser cut (single cases)

Appendix 3: Increase in $\mathrm{Ca}^{2+}$ signal in the neighboring cells following wounding (single cases)

Appendix 4: Spatial distribution of positive and negative coupling type of AS cell pairs

Appendix 5: $\quad$ F-actin staining in WT and $T M C^{\Delta}$ mutant embryos 


\section{Appendix 1: Anisotropy of AS cells (single cases)}

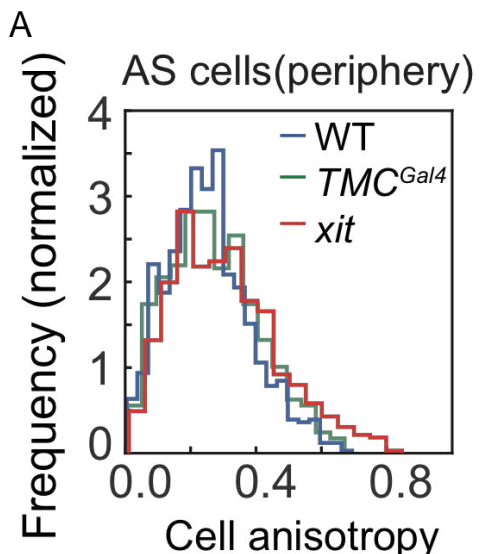

End\$f\$ermband\$etraction

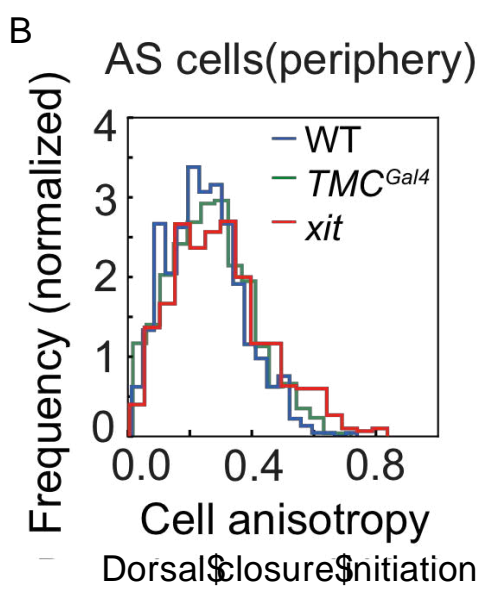

D

E 
Appendix 2: Displacement trajectories of junctions (single cases)

A

C

D

E
B

$\mathrm{F}$

Appendix 3: Increase in $\mathrm{Ca}+$ signal in the neighboring cells following wounding (single cases)
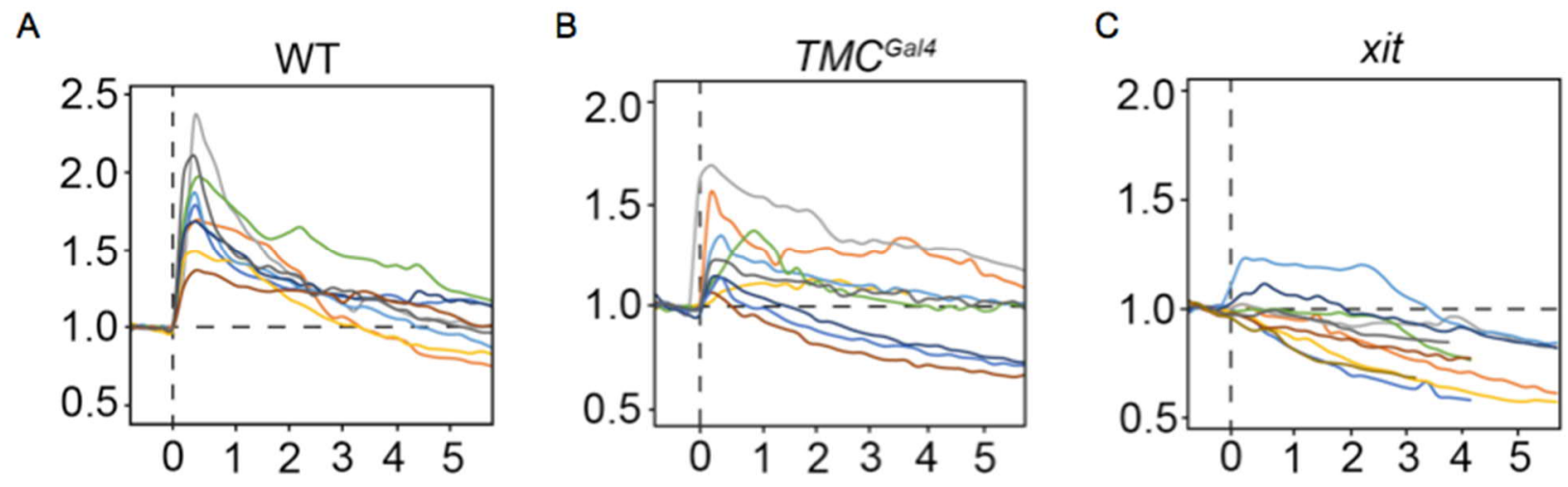
Appendix 4: Spatial distribution of positive and negative coupling type of AS cell pairs.

(Data overlapped from 8 wild-type, $7 T M C^{\Delta}$, and 7 xit embryos) 
Appendix 5: F-actin staining in WT and $T M C^{\Delta}$ mutant embryos

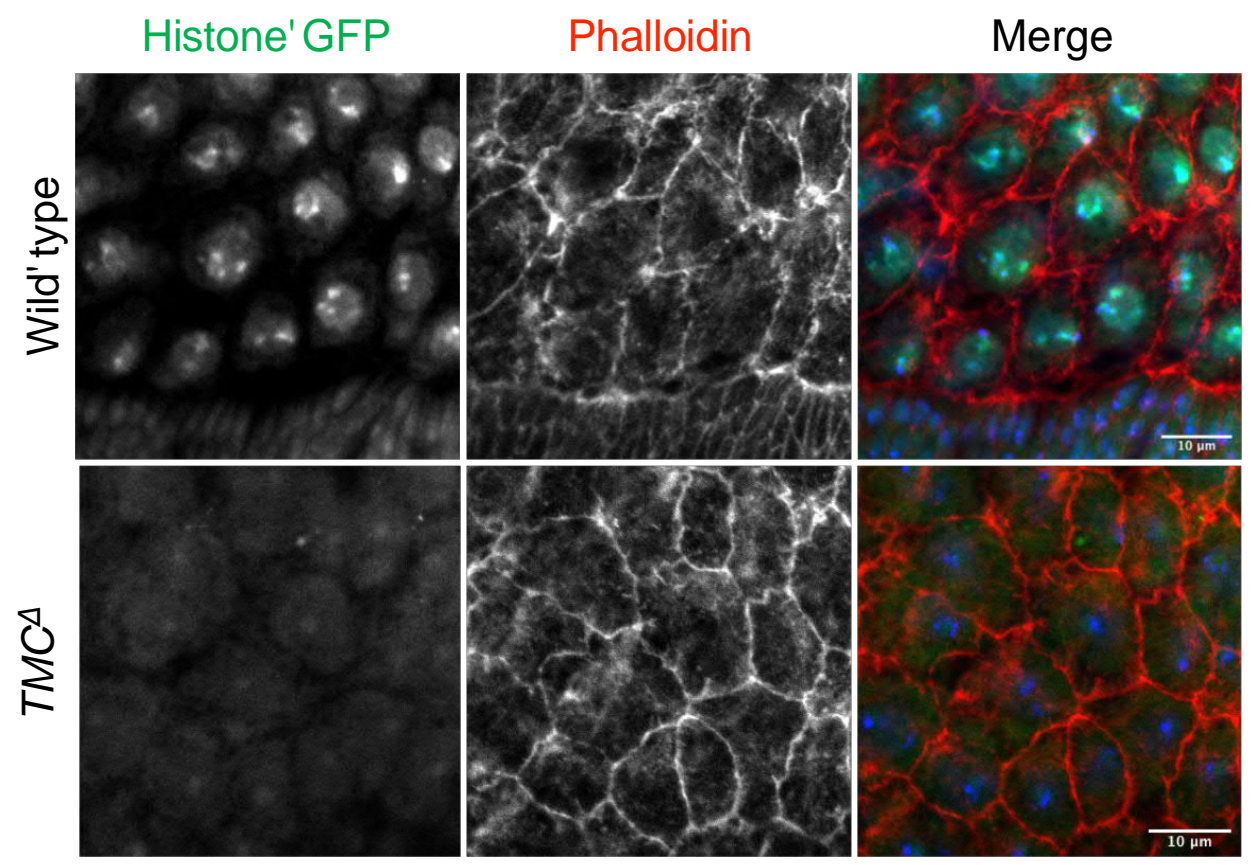

